\title{
A Ligand Electrochemical Parameter Approach to Molecular Design. $\sigma$-Donation, $\pi$-back Donation, and other Metrics in Ruthenium(II) Dinitrogen Complexes
}

By William J. Pietro and A. B. P. Lever*

Department of Chemistry, York University, Toronto, ON., Canada, M3J 1P3

blever@yorku.ca

\section{Supporting Information}

\begin{tabular}{|c|c|c|}
\hline & Cis isomer & Trans isomer \\
\hline п-back donation & 0.324 & 0.18 \\
\hline$\sigma$-Donation & 0.336 & 0.246 \\
\hline Net NBO charge on $\mathrm{N}_{2}$ & 0.0003 & 0.048 \\
\hline Net NBO charge on CO & 0.117 & 0.164 \\
\hline Net NBO charge on Ru & 0.247 & 0.190 \\
\hline N-N Bond order & 2.594 & 2.672 \\
\hline C-O Bond order & 2.250 & 2.256 \\
\hline Ru-N Bond order & 0.557 & 0.384 \\
\hline Ru-C Bond order & 1.166 & 1.168 \\
\hline $\begin{array}{l}\text { Mayer interfragment Bond } \\
\text { order }\end{array}$ & 0.992 & 0.698 \\
\hline \multicolumn{3}{|c|}{$\begin{array}{l}\mathrm{NB} \text { cis-CO isomer is that with a Chloride ligand trans to both } \mathrm{N}_{2} \\
\text { and } \mathrm{CO} \text {. The isomer with }(\mathrm{NH} 3) \text { trans to } \mathrm{CO} \text { has slightly differen } \\
\text { data }\end{array}$} \\
\hline
\end{tabular}




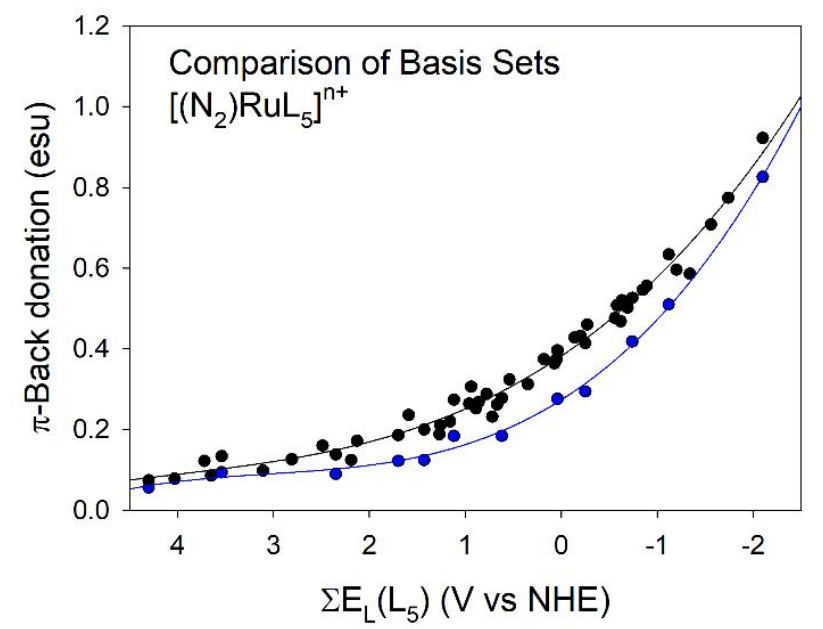

Figure S1 Calculation using lanl2dz for light elements up to an including fluoride and def2tzvp for heavier elements (black symbols), and using def2tzvp for all elements (blue symbols). Solid lines are cubic expressions to guide the eye and may not have theoretical significance

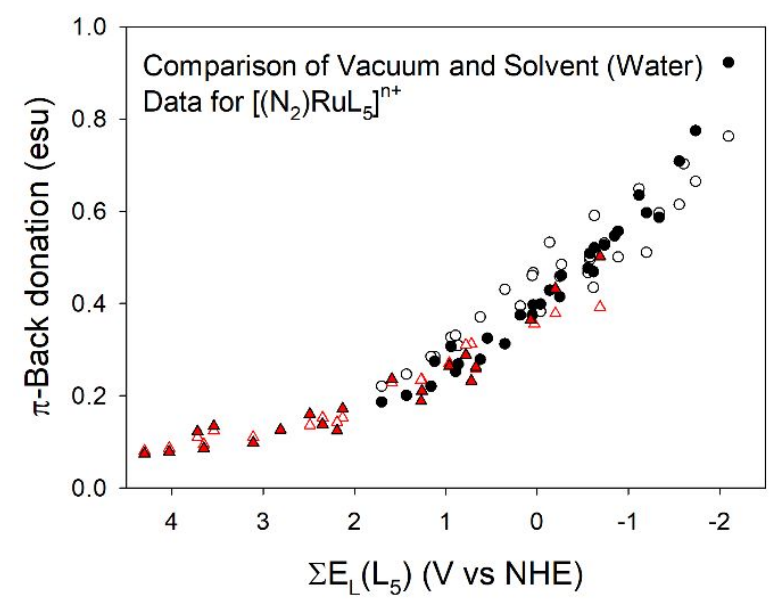

Figure S2 A comparison of data derived in vacuo with data derived using the Polarizable Continuum Model with water as solvent. Vacuo data use black circles and red triangles as in main text. Water data are cyan squares and triangles (for cis-CO data). 


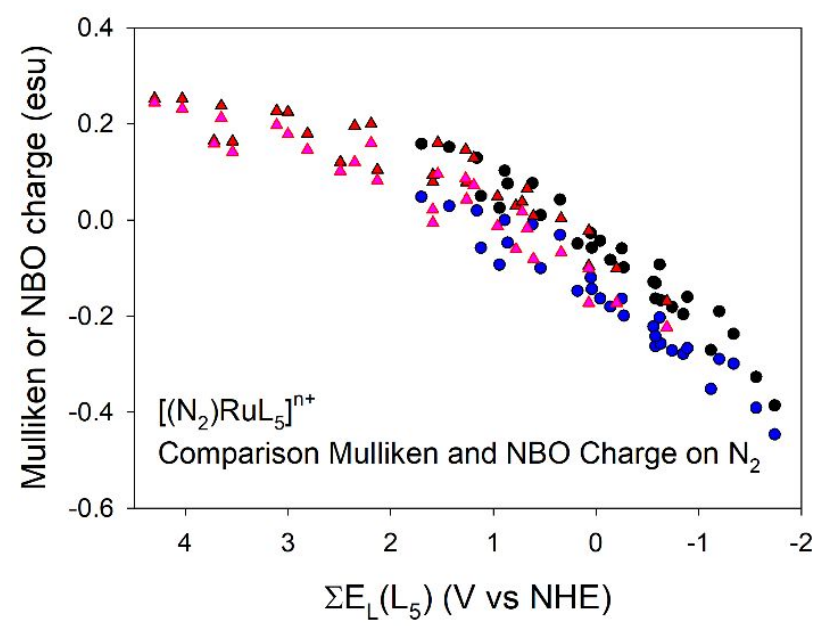

Figure S3 Comparison of Mulliken and NBO charge versus electron richness. Blue circles and pink triangles refer to Mulliken data. Black circles and red triangles refer to NBO charge.

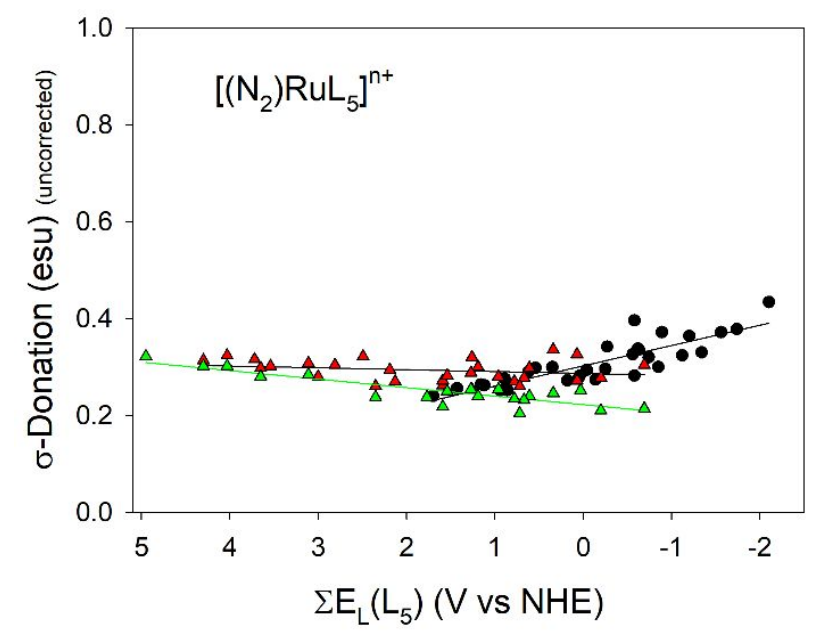

Figure S4 Correlation of electron richness with $\sigma$-donation uncorrected for electronic polarization.

This graph should be compared with Figure 3 in the main text. It is observed that the corrected data are less scattered than the uncorrected correlation as they should be. 


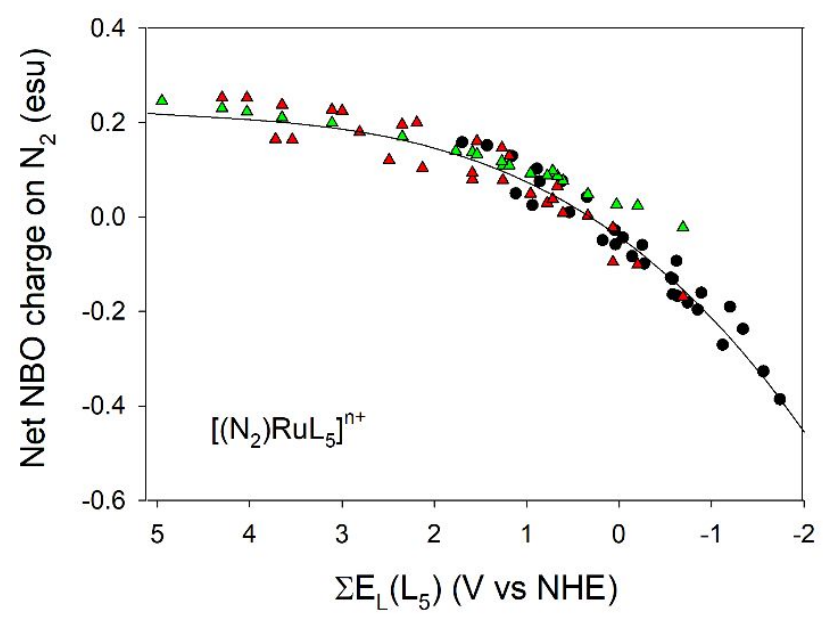

Figure S5 Correlation of net NBO charge on $\mathrm{N}_{2}$ with electron richness.

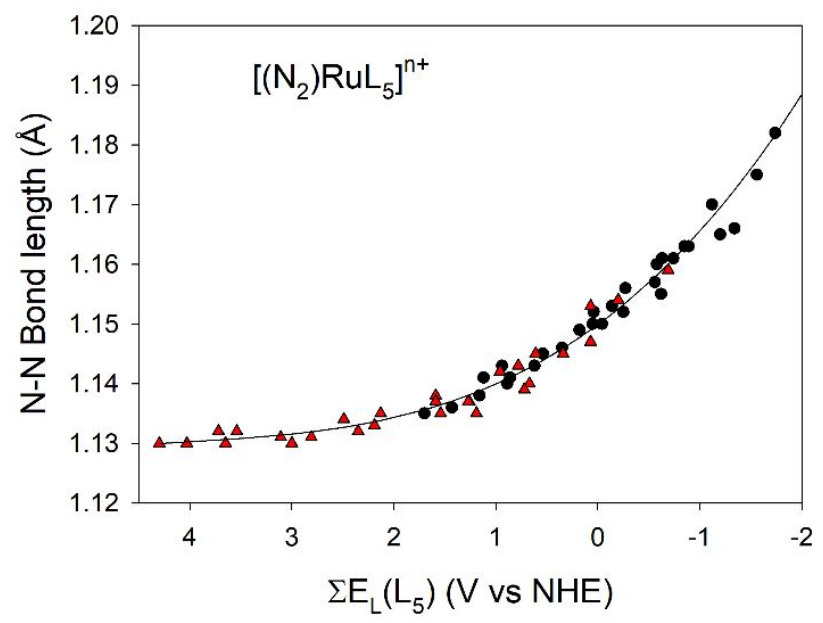

Figure $\mathbf{S 6}$ Correlation of $\mathrm{d}(\mathrm{NN})$ bond distance with electron richness. 


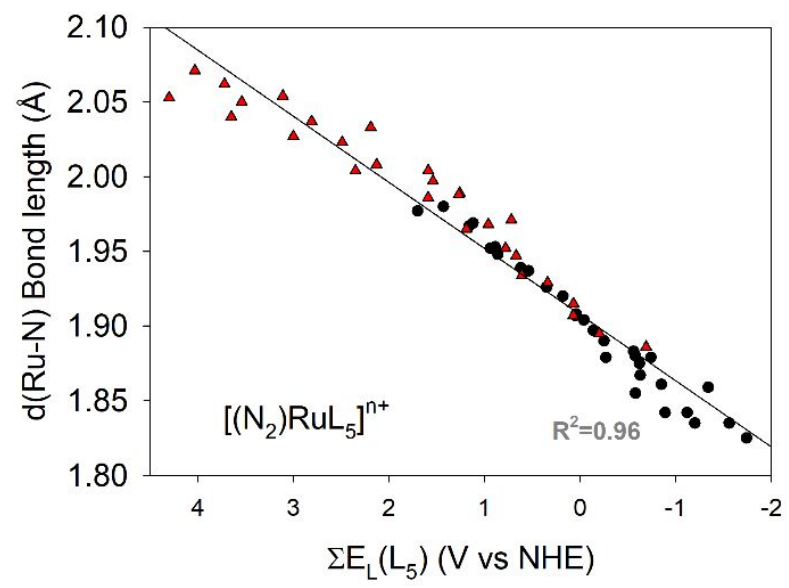

Figure $\mathbf{S 7}$ Correlation of $\mathrm{d}(\mathrm{RuN})$ bond distance with electron richness.

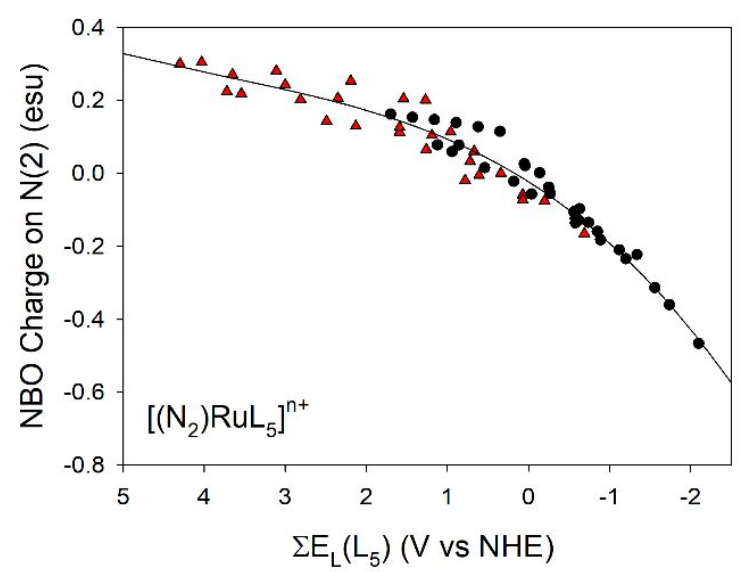

Figure S8 Correlation of NBO charge on N(2) with electron richness.

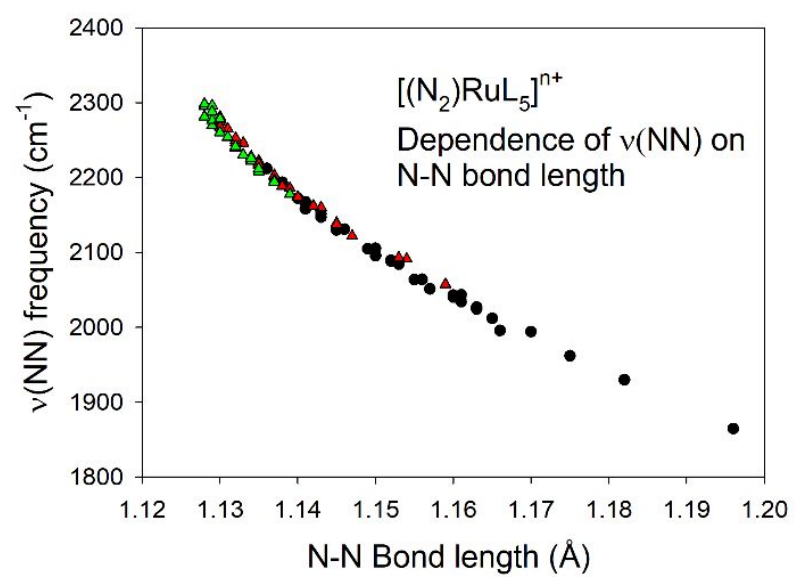

Figure S9. Correlation of $v(\mathrm{NN})$ stretching frequency with N-N Bond distance. 
Note overall spread of about 0.07 Angstrom over range of study of $\Sigma \mathrm{E}_{\mathrm{L}}\left(\mathrm{L}_{5}\right)$.

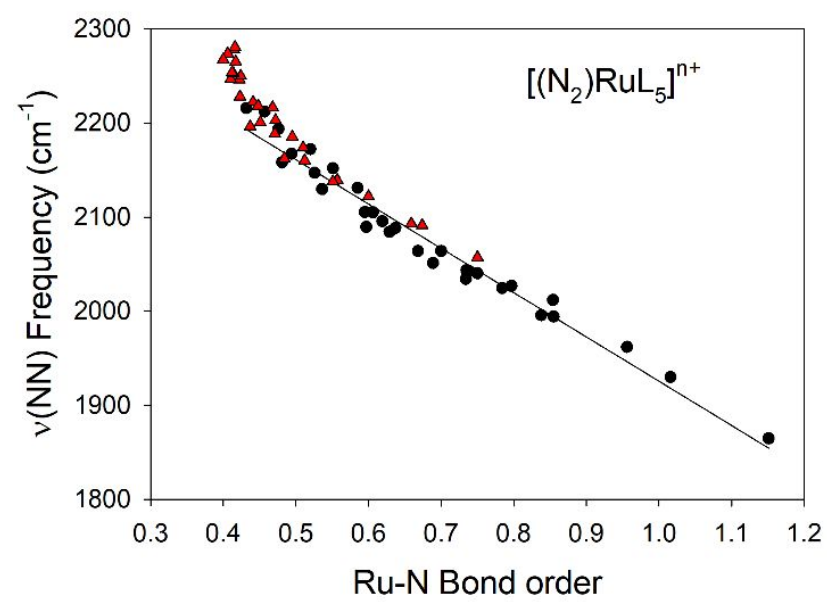

Figure S10. Correlation of $v(\mathrm{NN})$ stretching frequency with Ru-N bond order.

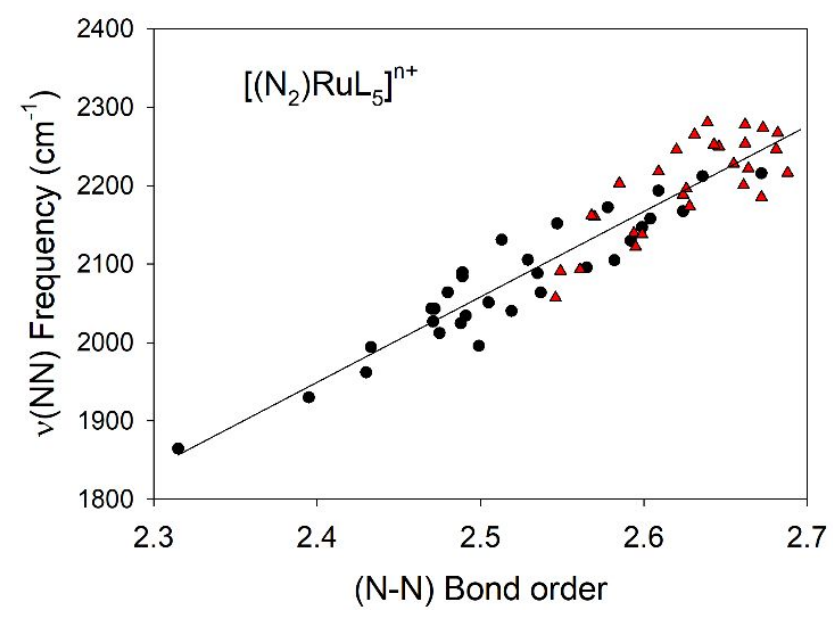

Figure S11 Correlation of $v(\mathrm{NN})$ stretching frequency with N-N bond order.

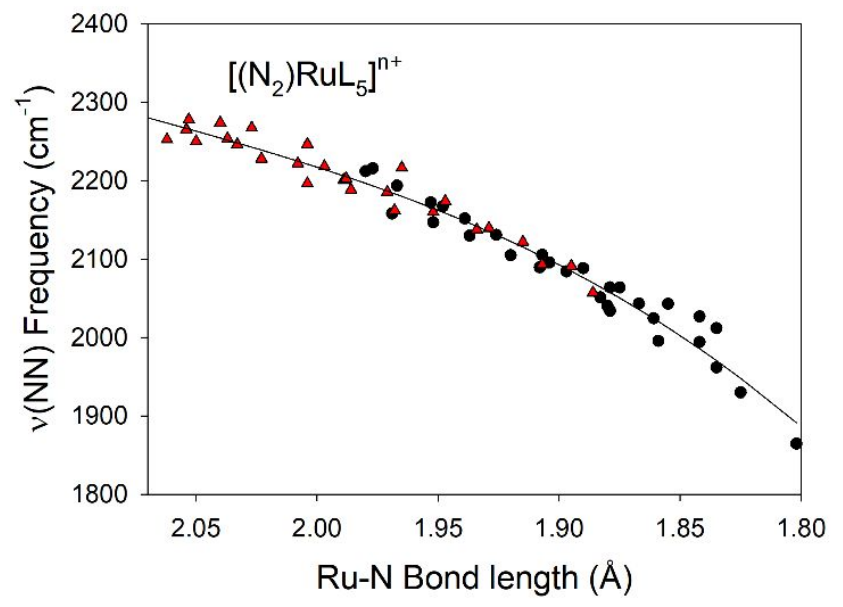

Figure $\mathbf{S 1 2}$ Correlation of $\mathrm{d}(\mathrm{Ru}-\mathrm{N})$ bond distance with $v(\mathrm{NN})$ stretching frequency. 


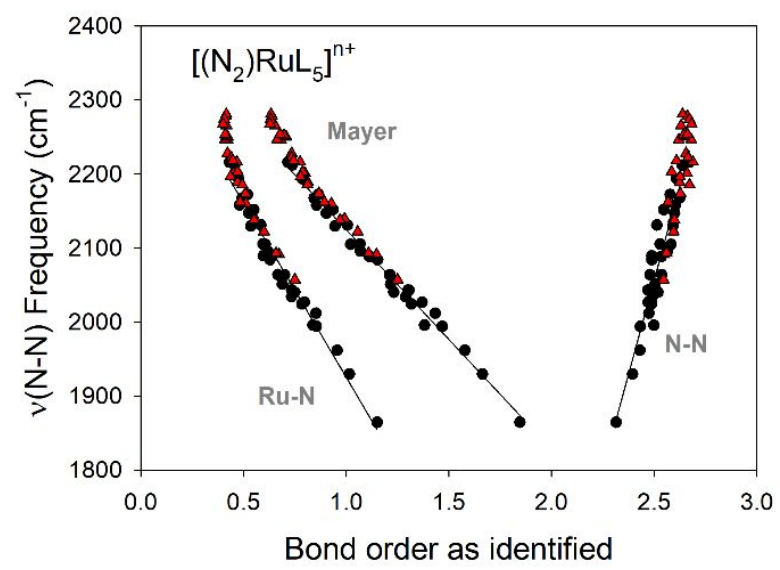

Figure S13 Comparison of Ru-N, N-N and Mayer interfragment bond order correlations with $\mathrm{v}(\mathrm{NN})$ stretching frequency.

This Figure reprises earlier data to show the change in slope associated with each bond order.

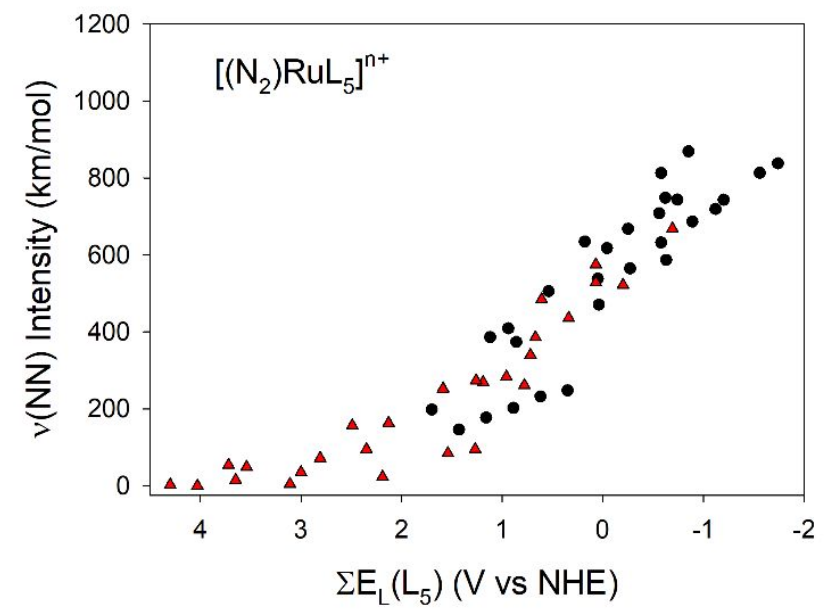

Figure S14 DFT derived infrared intensities $(\mathrm{km} / \mathrm{mol})$ of the $v(\mathrm{NN})$ stretching vibration versus electron richness. 


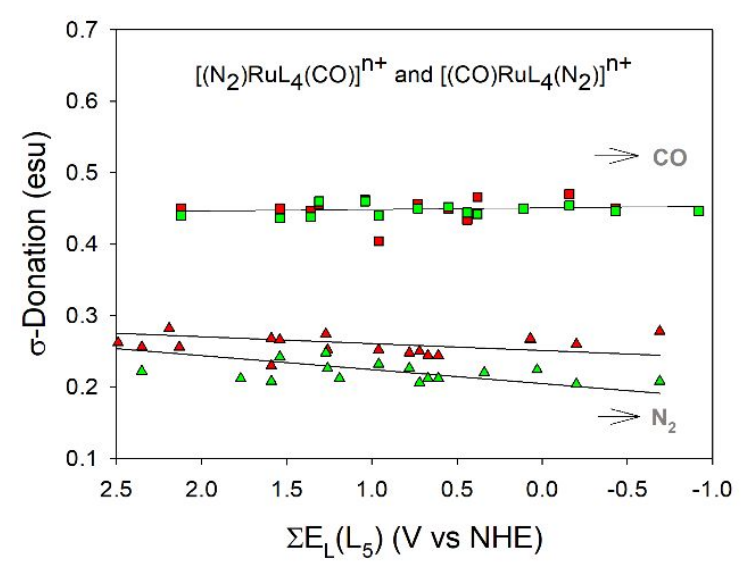

Figure S15 cis- and trans-Monocarbonyl species $\left.\left(\mathrm{N}_{2}\right) \mathrm{RuL}_{4}(\mathrm{CO})\right]^{\mathrm{n}+}$ species. Electron richness versus $\sigma$-donation, $\mathrm{Ru}$ to $\mathrm{CO}$ and to $\mathrm{N}_{2}$. Squares relate to the $\mathrm{CO}$ ligand, cisred and trans-green. [see Figure $\mathbf{1 6}$ in main text for correlation with $\pi$-back donation].

Abbreviations used: $a=\left(\mathrm{NH}_{3}\right) ; \mathrm{t}=$ trans; $(\mathrm{CH} 3 \mathrm{CN})$ is $\left(\mathrm{CH}_{3} \mathrm{CN}\right)$ i.e. multiple atoms not subscripted; (cCO) is one or more $\mathrm{CO}$ groups cis to the $\mathrm{RuN}_{2}$ vector; tco is a $\mathrm{CO}$ group trans to $\mathrm{RuN}_{2}$; Where multiple isomers exist, the ligand with least positive $\mathrm{E}_{\mathrm{L}}(\mathrm{L})$ value is trans to $\mathrm{RuN}_{2}$. Mer and fac have their usual meanings; the number of any specific group follows the name, so $(\mathrm{CH} 3 \mathrm{CN}) 4$ would signify $4(\mathrm{CH} 3 \mathrm{CN})$ groups. Energies are cited in Hartrees.

\section{Regular Files. No CO groups}

\begin{tabular}{llll}
8 & & & \\
(N2)RuF2Cl3 & \multicolumn{2}{l}{$\mathrm{E}(\mathrm{RB} 3 \mathrm{LYP})$} & $=-1784.86554314$ \\
7 & 1.004988 & 0.043468 & -0.088784 \\
7 & 2.178849 & 0.065502 & -0.140156 \\
44 & -0.827866 & -0.023621 & -0.04366 \\
17 & -0.956781 & 1.730267 & -1.901966 \\
17 & -0.88453 & 1.83545 & 1.832768 \\
17 & -0.73531 & -1.894861 & 1.69881 \\
9 & -0.861892 & -1.456792 & -1.483899 \\
9 & -2.842637 & -0.115908 & -0.015596
\end{tabular}




\begin{tabular}{|c|c|c|c|}
\hline & $\mathrm{u}(\mathrm{NH} 3) 2 \mathrm{~F} 3$ & $E(R$ & $3 \mathrm{LYP})=-617.356370283$ \\
\hline 7 & -2.270642 & 1.065369 & 1.547893 \\
\hline 7 & -3.300907 & 0.7423 & 1.998965 \\
\hline 44 & -0.647838 & 1.575024 & 0.840129 \\
\hline 9 & 1.132999 & 2.138862 & 0.065286 \\
\hline 9 & -1.246001 & 1.116001 & -1.080709 \\
\hline 7 & 0.142379 & -0.361724 & 0.544721 \\
\hline 1 & 0.002609 & -1.077048 & 1.254542 \\
\hline 1 & 1.125841 & -0.113713 & 0.404172 \\
\hline 1 & -0.335044 & -0.539984 & -0.350054 \\
\hline 7 & -0.952311 & 3.663715 & 0.923144 \\
\hline 1 & -0.191364 & 3.954403 & 0.302529 \\
\hline 1 & -1.85642 & 4.054285 & 0.668157 \\
\hline 9 & 0.096577 & 2.078199 & 2.697002 \\
\hline 1 & -0.696099 & 3.774574 & 1.914196 \\
\hline
\end{tabular}

\begin{tabular}{|c|c|c|c|}
\hline & (NH3)3F2 & & LYYP $=$ \\
\hline 7 & -1.88503 & -0.141675 & 1.342718 \\
\hline 7 & -2.151056 & -0.182355 & 2.471775 \\
\hline 44 & -1.471953 & -0.081079 & -0.476496 \\
\hline & -1.165807 & -0.062987 & -2.478496 \\
\hline & 0.421437 & 0.500549 & -0.097321 \\
\hline & -0.546233 & -1.989377 & -0.57532 \\
\hline 1 & -0.907102 & -2.800966 & -0.072854 \\
\hline 1 & 0.3694 & -1.697196 & -0.207355 \\
\hline 1 & -0.469886 & -2.165492 & -1.582408 \\
\hline 7 & -3.397759 & -0.686779 & -1.238184 \\
\hline 1 & -3.11983 & -0.636516 & -2.229302 \\
\hline 1 & -4.162672 & -0.040626 & -1.035311 \\
\hline 1 & -3.682795 & -1.633651 & -0.981462 \\
\hline 7 & -1.748296 & 2.011659 & -0.710998 \\
\hline 1 & -2.495254 & 2.518919 & -0.235398 \\
\hline 1 & -0.822428 & 2.290907 & -0.358342 \\
\hline & -1.782471 & 2.146109 & -1.726562 \\
\hline
\end{tabular}

\footnotetext{
29

(N2)Ru-(Acac)-(NH3)3 $\quad E(R B 3 L Y P)=-719.234291362$

$\begin{array}{llll}7 & 1.585155 & 2.056076 & 0.418502\end{array}$

$\begin{array}{llll}7 & 1.246385 & 2.904369 & -0.280036\end{array}$

$\begin{array}{llll}44 & 2.137871 & 0.621627 & 1.546864\end{array}$

$\begin{array}{llll}8 & 2.748607 & -0.859988 & 2.800296\end{array}$ 


$\begin{array}{llll}8 & 1.615602 & -0.745631 & 0.109684 \\ 7 & 2.741591 & 1.883757 & 3.184742 \\ 7 & 4.108693 & 0.597558 & 0.694215 \\ 6 & 2.701155 & -2.162589 & 2.558538 \\ 6 & 1.726562 & -2.051099 & 0.218395 \\ 6 & 2.225546 & -2.73494 & 1.36099 \\ 6 & 3.204111 & -3.037194 & 3.684382 \\ 6 & 1.298138 & -2.837094 & -1.001256 \\ 1 & 2.245803 & -3.816556 & 1.300485 \\ 1 & 3.154443 & -4.09899 & 3.432231 \\ 1 & 2.611108 & -2.860832 & 4.591071 \\ 1 & 4.242682 & -2.7786 & 3.927218 \\ 1 & 1.016046 & -3.862681 & -0.747227 \\ 1 & 0.462316 & -2.335729 & -1.498785 \\ 1 & 2.126014 & -2.88443 & -1.722811 \\ 7 & 0.154555 & 0.366768 & 2.329832 \\ 1 & 0.189224 & -0.21137 & 3.175897 \\ 1 & 1.981436 & 2.445598 & 3.579216 \\ 1 & 3.509735 & 2.52201 & 2.957374 \\ 1 & -0.345402 & 1.236414 & 2.536088 \\ 1 & -0.365091 & -0.13804 & 1.602812 \\ 1 & 3.062853 & 1.208225 & 3.889786 \\ 1 & 4.534696 & 1.517834 & 0.551703 \\ 1 & 4.010746 & 0.143741 & -0.221075 \\ 1 & 4.72399 & 0.027965 & 1.284522\end{array}$

16

(N2)Ru-(NH3)-(CH3CN)F3 E(RB3LYP) $=-693.525498033$

$\begin{array}{llll}7 & -1.890972 & -0.548302 & -0.279289 \\ 7 & -3.036352 & -0.668246 & -0.443587 \\ 44 & -0.054311 & -0.402539 & -0.020197 \\ 9 & 1.943578 & -0.437713 & 0.255301 \\ 9 & 0.30304 & -0.496164 & -2.022661 \\ 9 & -0.25569 & -0.621707 & 2.001564 \\ 7 & 0.349016 & -2.46503 & 0.056235 \\ 1 & -0.056151 & -2.822598 & 0.922111 \\ 1 & 1.365746 & -2.313203 & 0.133712 \\ 1 & 0.081222 & -2.975749 & -0.783243 \\ 7 & -0.095116 & 1.542753 & -0.003372 \\ 6 & -0.047324 & 2.716265 & 0.0062 \\ 6 & 0.057591 & 4.173831 & 0.020622 \\ 1 & 0.278659 & 4.542843 & 1.031504 \\ 1 & 0.860334 & 4.510789 & -0.649096 \\ 1 & -0.880976 & 4.63557 & -0.31373\end{array}$




\begin{tabular}{|c|c|c|c|}
\hline \multicolumn{4}{|r|}{$3 L Y P)=-18$} \\
\hline 7 & -1.188406 & 0.150961 & 3.218038 \\
\hline 7 & -1.094213 & 0.411559 & 4.334351 \\
\hline 44 & -1.345011 & -0.280759 & 1.370761 \\
\hline 17 & 0.811282 & -1.442108 & 1.508672 \\
\hline 17 & -3.492224 & 0.90391 & 1.324914 \\
\hline 17 & -1.544411 & -0.829381 & -0.975098 \\
\hline 7 & -2.286117 & -1.97476 & 1.759387 \\
\hline 6 & -2.846266 & -2.985945 & 1.917636 \\
\hline 6 & -3.549273 & -4.258004 & 2.05599 \\
\hline 1 & -4.256305 & -4.223201 & 2.893983 \\
\hline 1 & -4.109415 & -4.477059 & 1.138631 \\
\hline 1 & -2.838169 & -5.074581 & 2.23189 \\
\hline 7 & -0.417669 & 1.373949 & 0.814759 \\
\hline 6 & 0.122339 & 2.328128 & 0.415678 \\
\hline 6 & 0.79016 & 3.500851 & -0.141206 \\
\hline 1 & 0.114662 & 4.365175 & -0.144344 \\
\hline 1 & 1.682296 & 3.755832 & 0.443984 \\
\hline 1 & 1.099329 & 3.298413 & -1.174016 \\
\hline
\end{tabular}

14

(N2)Ru(NH3)2FacCl3 $\quad E(R B 3 L Y P)=-1698.51875118$

$\begin{array}{llll}7 & 0.130134 & 1.143556 & -1.813169\end{array}$

$\begin{array}{llll}7 & 0.217575 & 1.72332 & -2.813907\end{array}$

$\begin{array}{llll}44 & -0.005097 & 0.155122 & -0.248822\end{array}$

$\begin{array}{llll}17 & 1.767693 & 1.49267 & 0.777384\end{array}$

$\begin{array}{llll}17 & -0.162497 & -1.473083 & 1.629933\end{array}$

$\begin{array}{llll}17 & -1.948668 & 1.470656 & 0.442255\end{array}$

$\begin{array}{llll}1 & 1.582652 & -1.864377 & -0.07426\end{array}$

$\begin{array}{llll}1 & 1.800615 & -1.3794 & -1.693398\end{array}$

$\begin{array}{llll}7 & 1.685969 & -1.078282 & -0.725549\end{array}$

$\begin{array}{llll}7 & 2.445174 & -0.453205 & -0.418298\end{array}$

$\begin{array}{llll}7 & -1.570655 & -1.097304 & -1.018033\end{array}$

$\begin{array}{llll}1 & -2.378786 & -0.477391 & -0.863487\end{array}$

$1 \quad-1.501287 \quad-1.408726 \quad-1.986921$

$\begin{array}{llll}1 & -1.5828 & -1.876117 & -0.350074\end{array}$

23

(N2)Ru(NH3)5 $\quad E(R B 3 L Y P)=-486.833535062$

$\begin{array}{llll}7 & -1.022897 & -1.50325 & -0.035458\end{array}$

$\begin{array}{llll}7 & -1.673521 & -2.446291 & -0.055403\end{array}$

$\begin{array}{llll}44 & 0.06769 & 0.0837 & 0.002347\end{array}$ 


$\begin{array}{llll}1 & -2.50948 & 0.793178 & -0.452868 \\ 7 & -1.732147 & 1.317131 & -0.031383 \\ 1 & -2.043462 & 1.591078 & 0.908527 \\ 1 & -1.627819 & 2.185628 & -0.57021 \\ 7 & 0.112753 & 0.10096 & -2.180939 \\ 7 & 0.011581 & 0.036704 & 2.182504 \\ 7 & 1.868811 & -1.150695 & 0.028671 \\ 7 & 1.294204 & 1.888664 & 0.052045 \\ 1 & -0.577694 & 0.74322 & -2.58831 \\ 1 & -0.11621 & -0.835362 & -2.53745 \\ 1 & 1.024788 & 0.355113 & -2.579723 \\ 1 & -0.954951 & -0.050633 & 2.520692 \\ 1 & 0.418002 & 0.862393 & 2.638274 \\ 1 & 0.51198 & -0.783344 & 2.546968 \\ 1 & 1.612332 & -2.145242 & 0.067407 \\ 1 & 2.475747 & -0.969861 & 0.837698 \\ 1 & 2.45138 & -1.031936 & -0.809041 \\ 1 & 1.415993 & 2.305696 & -0.879086 \\ 1 & 0.88018 & 2.618842 & 0.645 \\ 1 & 2.238216 & 1.713506 & 0.418122\end{array}$

$\begin{array}{llll}35 & & & \\ \text { (N2)Ru(Acac)2NH3 } & E(\mathrm{RB} 3 \mathrm{LY})= & -951.467115054 \\ 7 & -1.009483 & 1.383873 & 2.054809 \\ 7 & -1.807344 & 1.031616 & 2.80793 \\ 44 & 0.32351 & 1.944983 & 0.837685 \\ 8 & 1.742829 & 2.576363 & -0.493749 \\ 8 & 1.647467 & 0.569634 & 1.600497 \\ 6 & 3.014257 & 2.244141 & -0.485635 \\ 6 & 3.867117 & 2.988499 & -1.495176 \\ 1 & 4.899888 & 2.627995 & -1.507793 \\ 1 & 3.434778 & 2.885467 & -2.498409 \\ 1 & 3.86505 & 4.059346 & -1.253147 \\ 6 & 3.600644 & 1.280779 & 0.36205 \\ 6 & 2.922292 & 0.502929 & 1.339246 \\ 6 & 3.696335 & -0.497312 & 2.177411 \\ 1 & 4.711611 & -0.653641 & 1.800771 \\ 1 & 3.163165 & -1.454879 & 2.203515 \\ 1 & 4.66715 & 1.116743 & 0.258479 \\ 1 & 3.755604 & -0.131945 & 3.211455 \\ 7 & -0.345352 & 0.622829 & -0.695309 \\ 1 & 0.389249 & 0.58247 & -1.407078 \\ 1 & -0.56639 & -0.318012 & -0.362843 \\ 1 & -1.176465 & 1.076615 & -1.08772 \\ 8 & -0.949918 & 3.294196 & -0.027375\end{array}$




$\begin{array}{llll}8 & 1.054009 & 3.324021 & 2.16946 \\ 6 & -1.02332 & 4.557494 & 0.311596 \\ 6 & -1.997304 & 5.376733 & -0.514862 \\ 1 & -2.038325 & 6.419491 & -0.187671 \\ 1 & -1.698915 & 5.34485 & -1.570722 \\ 1 & -3.001562 & 4.939528 & -0.445948 \\ 6 & -0.287237 & 5.175449 & 1.349103 \\ 6 & 0.679348 & 4.574296 & 2.197424 \\ 6 & 1.374967 & 5.41622 & 3.251818 \\ 1 & 1.029833 & 6.454148 & 3.245473 \\ 1 & 2.458866 & 5.395364 & 3.08146 \\ 1 & -0.474095 & 6.231464 & 1.507858 \\ 1 & 1.19555 & 4.98263 & 4.243643\end{array}$

32

\begin{tabular}{llll} 
(N2)Ru(Acac)2Cl & \multicolumn{2}{l}{$E($ RB3LYP $)=-1355.21089543$} \\
7 & -0.15043 & 1.590621 & 0.931666 \\
7 & -0.986912 & 0.790671 & 0.936069 \\
44 & 1.210048 & 2.893069 & 0.924844 \\
17 & 2.95246 & 4.564479 & 0.917323 \\
8 & 2.505212 & 1.600681 & 1.882129 \\
6 & 2.855709 & 1.420043 & -1.114527 \\
6 & 3.214897 & 1.198258 & -2.57735 \\
1 & 3.973724 & 0.418311 & -2.703321 \\
1 & 2.312898 & 0.927174 & -3.140368 \\
1 & 3.592868 & 2.139439 & -2.998156 \\
6 & 3.58842 & 0.740742 & -0.108465 \\
6 & 3.395001 & 0.861081 & 1.290965 \\
6 & 4.290546 & 0.07472 & 2.238407 \\
1 & 4.965285 & -0.602694 & 1.703663 \\
1 & 3.670227 & -0.501725 & 2.93637 \\
1 & 4.384265 & 0.081705 & -0.4401 \\
1 & 4.885307 & 0.779277 & 2.834559 \\
8 & -0.030186 & 4.237725 & -0.03296 \\
8 & 0.604733 & 3.591789 & 2.771283 \\
6 & -0.727797 & 5.16092 & 0.558121 \\
6 & -1.476454 & 6.088643 & -0.38893 \\
1 & -2.132981 & 6.783686 & 0.145856 \\
1 & -0.747731 & 6.662254 & -0.976881 \\
1 & -2.06954 & 5.493016 & -1.094252 \\
6 & -0.834609 & 5.363842 & 1.957377 \\
6 & -0.182955 & 4.606978 & 2.963793 \\
6 & -0.384592 & 4.979638 & 4.426138 \\
1 & -1.131732 & 5.770744 & 4.552272 \\
1 & 0.57295 & 5.319038 & 4.842855
\end{tabular}




$\begin{array}{llll}1 & -1.458379 & 6.187945 & 2.288321 \\ 1 & -0.691397 & 4.091425 & 4.992764 \\ 8 & 1.874657 & 2.249636 & -0.921449\end{array}$

\begin{tabular}{llll}
32 & & & \\
(N2)Ru(Acac)2F & \multicolumn{2}{l}{$E($ RB $L Y Y)$} & $=$ \\
7 & -994.826348017 \\
7 & -0.173963 & 1.675599 & 1.205242 \\
7 & -1.042829 & 0.918829 & 1.345719 \\
44 & 1.233417 & 2.900044 & 0.9766 \\
9 & 2.719968 & 4.1885 & 0.734417 \\
8 & 1.773633 & 2.069292 & -0.830098 \\
8 & 2.559587 & 1.689967 & 1.987352 \\
6 & 2.745711 & 1.223367 & -0.994813 \\
6 & 3.025768 & 0.858748 & -2.44703 \\
1 & 3.80105 & 0.090077 & -2.538227 \\
1 & 2.101826 & 0.503942 & -2.921419 \\
1 & 3.34561 & 1.759363 & -2.987776 \\
6 & 3.532668 & 0.63994 & 0.030715 \\
6 & 3.415087 & 0.892928 & 1.421205 \\
6 & 4.357714 & 0.188784 & 2.388628 \\
1 & 5.024889 & -0.514208 & 1.877774 \\
1 & 3.771864 & -0.349117 & 3.145151 \\
1 & 4.309064 & -0.052395 & -0.27903 \\
1 & 4.959911 & 0.940656 & 2.915472 \\
8 & 0.028323 & 4.217244 & -0.05315 \\
8 & 0.81934 & 3.836834 & 2.764022 \\
6 & -0.54458 & 5.259574 & 0.46955 \\
6 & -1.284969 & 6.14042 & -0.528537 \\
1 & -1.818223 & 6.962315 & -0.038183 \\
1 & -0.56392 & 6.555636 & -1.245182 \\
1 & -1.997766 & 5.530749 & -1.098414 \\
6 & -0.531409 & 5.62534 & 1.839816 \\
6 & 0.130174 & 4.931395 & 2.884868 \\
6 & 0.058852 & 5.478646 & 4.304605 \\
1 & -0.576669 & 6.36842 & 4.373412 \\
1 & 1.072092 & 5.731513 & 4.643607 \\
1 & -1.06382 & 6.531258 & 2.111719 \\
1 & -0.326635 & 4.702257 & 4.97805 \\
& & &
\end{tabular}

\footnotetext{
35

(N2)Ru(Acac) $(\mathrm{CH} 3 \mathrm{CN}) 3 \quad \mathrm{E}(\mathrm{RB} 3 \mathrm{LYP})=-947.776371910$

$\begin{array}{llll}7 & 1.432721 & 1.63285 & -0.276757\end{array}$

$\begin{array}{llll}7 & 0.997987 & 2.175743 & -1.180907\end{array}$ 


$\begin{array}{llll}44 & 2.146016 & 0.640841 & 1.240969 \\ 8 & 2.882875 & -0.404206 & 2.818705 \\ 8 & 1.341134 & -1.054735 & 0.445023 \\ 7 & 2.959266 & 2.296124 & 2.058338 \\ 7 & 3.842564 & 0.239622 & 0.238987 \\ 6 & 2.749345 & -1.696584 & 3.023638 \\ 6 & 1.408001 & -2.25891 & 0.96144 \\ 6 & 3.447632 & 3.226498 & 2.567637 \\ 6 & 4.81695 & -0.023523 & -0.346777 \\ 6 & 2.063615 & -2.590411 & 2.171544 \\ 6 & 3.406291 & -2.198336 & 4.290744 \\ 6 & 0.706912 & -3.328379 & 0.153144 \\ 6 & 4.063066 & 4.385357 & 3.211288 \\ 6 & 6.029786 & -0.360972 & -1.087911 \\ 1 & 2.037816 & -3.630517 & 2.472271 \\ 1 & 3.282238 & -3.276483 & 4.418282 \\ 1 & 2.977998 & -1.683298 & 5.159813 \\ 1 & 4.477057 & -1.959938 & 4.273199 \\ 1 & 0.833517 & -4.3221 & 0.589818 \\ 1 & -0.36473 & -3.100737 & 0.088478 \\ 1 & 1.093628 & -3.332275 & -0.873128 \\ 1 & 4.604742 & 4.990948 & 2.475636 \\ 1 & 3.29876 & 5.014287 & 3.682006 \\ 1 & 4.769529 & 4.06021 & 3.983669 \\ 1 & 5.994036 & -1.404679 & -1.420565 \\ 1 & 6.128936 & 0.281362 & -1.970486 \\ 1 & 6.915339 & -0.226614 & -0.456393 \\ 6 & -1.805468 & 1.164485 & 3.612502 \\ 1 & -1.963208 & 0.2945 & 4.260243 \\ 1 & -1.785199 & 2.064744 & 4.237169 \\ 1 & -2.65107 & 1.239896 & 2.919244 \\ 6 & -0.55382 & 1.025075 & 2.87218 \\ 7 & 0.441315 & 0.90491 & 2.274591\end{array}$

\begin{tabular}{llll}
23 & & & \\
$(\mathrm{~N} 2) \mathrm{Ru}(\mathrm{CH} 3 \mathrm{CN}) 3 \mathrm{~F} 2$ & \multicolumn{3}{c}{$\mathrm{E}(\mathrm{RB} 3 \mathrm{LYP})=-802.508602245$} \\
7 & 1.391477 & -2.208247 & 0.987114 \\
7 & 1.836113 & -3.236748 & 1.242397 \\
44 & 0.657988 & -0.476422 & 0.601878 \\
9 & 1.094829 & 0.104152 & 2.446447 \\
9 & -0.086722 & 1.31745 & 0.244927 \\
7 & 2.429691 & 0.267588 & 0.077576 \\
6 & 3.439244 & 0.814835 & -0.126947 \\
6 & 4.691474 & 1.537353 & -0.334548 \\
1 & 5.29592 & 1.060873 & -1.115065
\end{tabular}




$\begin{array}{llll}1 & 4.483099 & 2.571085 & -0.634013 \\ 1 & 5.272426 & 1.556899 & 0.594891 \\ 7 & 0.169408 & -0.93632 & -1.282197 \\ 6 & -0.145473 & -1.126511 & -2.393608 \\ 6 & -0.54681 & -1.338867 & -3.78334 \\ 1 & -1.234782 & -2.189119 & -3.863806 \\ 1 & -1.05223 & -0.446748 & -4.172365 \\ 1 & 0.327554 & -1.542783 & -4.413147 \\ 7 & -1.138411 & -0.97305 & 1.305493 \\ 6 & -2.179373 & -1.142969 & 1.803571 \\ 6 & -3.469673 & -1.312344 & 2.466684 \\ 1 & -3.513738 & -2.277291 & 2.984934 \\ 1 & -3.614085 & -0.514539 & 3.204521 \\ 1 & -4.289093 & -1.266863 & 1.739978\end{array}$

\begin{tabular}{|c|c|c|c|}
\hline \multicolumn{4}{|c|}{$\begin{array}{l}23 \\
(\mathrm{~N} 2) \mathrm{Ru}(\mathrm{CH} 3 \mathrm{CN}) 3 \mathrm{Cl} 2\end{array} \mathrm{E}(\mathrm{RB} 3 \mathrm{LYP})=-1523.27914200$} \\
\hline 7 & -0.318608 & 1.420445 & 2.377634 \\
\hline 7 & -0.071386 & 1.862561 & 3.404587 \\
\hline 44 & -0.73169 & 0.656309 & 0.646757 \\
\hline 17 & -0.079176 & -1.478561 & 1.511091 \\
\hline 17 & -1.245191 & -0.271963 & -1.49675 \\
\hline 7 & -1.295088 & 2.429529 & -0.132077 \\
\hline 6 & -1.636822 & 3.421393 & -0.647716 \\
\hline 6 & -2.073416 & 4.635784 & -1.334291 \\
\hline 1 & -1.261247 & 5.371066 & -1.376087 \\
\hline 1 & -2.378738 & 4.396296 & -2.359762 \\
\hline 1 & -2.925272 & 5.090322 & -0.814879 \\
\hline 7 & -2.598929 & 0.212775 & 1.200548 \\
\hline 6 & -3.664943 & -0.162968 & 1.486169 \\
\hline 6 & -4.977755 & -0.705884 & 1.824324 \\
\hline 1 & -4.861259 & -1.604582 & 2.441128 \\
\hline 1 & -5.575313 & 0.0258 & 2.380107 \\
\hline 1 & -5.517424 & -0.980248 & 0.9105 \\
\hline 6 & 3.611282 & 0.824796 & -0.89669 \\
\hline 1 & 4.166855 & 1.737008 & -0.650571 \\
\hline 1 & 3.60073 & 0.70185 & -1.985889 \\
\hline 1 & 4.132651 & -0.032916 & -0.456039 \\
\hline 6 & 2.24465 & 0.889272 & -0.386074 \\
\hline 7 & 1.149069 & 0.879767 & 0.012368 \\
\hline
\end{tabular}

33

(N2)Ru(CH3CN)5 $\quad E(R B 3 L Y P)=-867.797156642$

$\begin{array}{llll}7 & 0.003831 & 0.003596 & -2.181143\end{array}$ 


$\begin{array}{llll}7 & 0.005779 & 0.005564 & -3.316234 \\ 44 & 0.000521 & -2.5 \mathrm{E}-05 & -0.204489 \\ 7 & -1.447842 & 1.417274 & -0.205942 \\ 6 & -2.282084 & 2.233635 & -0.223178 \\ 6 & -3.325405 & 3.254927 & -0.258328 \\ 1 & -3.585651 & 3.496858 & -1.295648 \\ 1 & -4.227985 & 2.899685 & 0.252446 \\ 1 & -2.982779 & 4.171157 & 0.236527 \\ 7 & 1.448769 & -1.417424 & -0.206384 \\ 6 & 2.282907 & -2.233896 & -0.22323 \\ 6 & 3.326011 & -3.255428 & -0.257691 \\ 1 & 3.559956 & -3.524987 & -1.29446 \\ 1 & 4.240968 & -2.886316 & 0.220161 \\ 1 & 2.997087 & -4.158124 & 0.27024 \\ 7 & -0.002339 & -0.004119 & 1.816345 \\ 6 & -0.003269 & -0.00688 & 2.983204 \\ 6 & -0.004116 & -0.010677 & 4.443497 \\ 1 & -0.270851 & -1.003805 & 4.823561 \\ 1 & 0.988167 & 0.253492 & 4.827542 \\ 1 & -0.73021 & 0.715218 & 4.827886 \\ 7 & 1.417742 & 1.448395 & -0.201047 \\ 6 & 2.233858 & 2.282935 & -0.215067 \\ 6 & 3.254768 & 3.32676 & -0.246009 \\ 1 & 2.893464 & 4.232312 & 0.255159 \\ 1 & 4.16588 & 2.988021 & 0.26083 \\ 1 & 3.507607 & 3.580529 & -1.282331 \\ 7 & -1.417224 & -1.447943 & -0.211389 \\ 6 & -2.234483 & -2.281242 & -0.231412 \\ 6 & -3.25723 & -3.323019 & -0.269936 \\ 1 & -3.606403 & -3.475045 & -1.298156 \\ 1 & -2.853549 & -4.271422 & 0.103335 \\ 1 & -4.115881 & -3.041449 & 0.350714\end{array}$

\begin{tabular}{|c|c|c|c|}
\hline \multicolumn{4}{|c|}{$(\mathrm{N} 2) \mathrm{Ru}(\mathrm{CH} 3 \mathrm{CN}) 2 \mathrm{~F} 3 \quad \mathrm{E}(\mathrm{RB} 3 \mathrm{LYP})=-769.684309890$} \\
\hline 7 & -1.06513 & 0.074983 & 3.022982 \\
\hline 7 & -0.735501 & 0.020243 & 4.133652 \\
\hline 44 & -1.601308 & 0.16381 & 1.223004 \\
\hline 9 & 0.195376 & -0.560805 & 0.594285 \\
\hline 9 & -3.428401 & 0.893299 & 1.750267 \\
\hline 9 & -2.169789 & 0.256408 & -0.679885 \\
\hline 7 & -2.337943 & -1.657245 & 1.250046 \\
\hline 6 & -2.806601 & -2.72695 & 1.186268 \\
\hline 6 & -3.409028 & -4.052024 & 1.066782 \\
\hline 1 & -3.044761 & -4.719951 & 1.857847 \\
\hline
\end{tabular}




$\begin{array}{llll}1 & -4.502187 & -3.988662 & 1.144233 \\ 1 & -3.157882 & -4.498644 & 0.09625 \\ 7 & -0.917074 & 1.992703 & 1.007348 \\ 6 & -0.523579 & 3.073482 & 0.794974 \\ 6 & -0.036662 & 4.415613 & 0.487625 \\ 1 & 0.133604 & 4.517653 & -0.591732 \\ 1 & -0.766062 & 5.176251 & 0.794703 \\ 1 & 0.91043 & 4.615275 & 1.005307\end{array}$

\begin{tabular}{llll}
25 & \multicolumn{4}{c}{ (N) } \\
(N2)Ru(NH3)4(CH3CN) & $E(R B 3 L Y P)=-563.033955016$ \\
7 & -0.634534 & 0.667014 & 1.456574 \\
7 & -0.855714 & -0.251323 & 2.100058 \\
44 & -0.275099 & 2.212711 & 0.342642 \\
7 & 0.951975 & 3.049091 & 1.92922 \\
1 & 0.43775 & .064196 & 2.818616 \\
1 & 1.270713 & 4.008311 & 1.749296 \\
1 & 1.790939 & 2.479454 & 2.093464 \\
7 & 1.453324 & 1.199861 & -0.504684 \\
1 & 1.244871 & 0.920183 & -1.47031 \\
1 & 1.657808 & 0.33786 & 0.015191 \\
1 & 2.317876 & 1.753367 & -0.524535 \\
7 & -2.035638 & 3.20752 & 1.141451 \\
1 & -2.709484 & 3.385917 & 0.38766 \\
1 & -2.508092 & 2.600381 & 1.822083 \\
1 & -1.852272 & 4.10169 & 1.611486 \\
7 & 0.111628 & 3.932592 & -0.92439 \\
1 & -0.296015 & 4.803475 & -0.563337 \\
1 & 1.111019 & 4.112313 & -1.077609 \\
1 & -0.308666 & 3.765541 & -1.84654 \\
7 & -1.423158 & 1.434997 & -1.139446 \\
6 & -2.099048 & 0.933152 & -1.956439 \\
6 & -2.944663 & 0.289159 & -2.959833 \\
1 & -2.615043 & -0.743169 & -3.129839 \\
1 & -3.988338 & 0.266322 & -2.623234 \\
1 & -2.898733 & 0.829925 & -3.912744
\end{tabular}

11 (N2)RuNH3Cl4 $\quad E(R B 3 L Y P)=-2102.15731097$

$\begin{array}{llll}7 & -1.025803 & -1.901106 & -0.580849 \\ 7 & -1.538205 & -2.85113 & -1.012946 \\ 44 & -0.21537 & -0.373085 & 0.053323 \\ 17 & -2.472347 & 0.683354 & 0.230689 \\ 17 & -0.170697 & -1.295263 & 2.335419\end{array}$




$\begin{array}{llll}17 & 1.988685 & -1.309913 & -0.663962 \\ 17 & 0.883603 & 1.813845 & 0.668234 \\ 7 & -0.176415 & 0.56717 & -1.851922 \\ 1 & 0.409946 & 0.007212 & -2.472118 \\ 1 & 0.274502 & 1.447885 & -1.571316 \\ 1 & -1.140095 & 0.701488 & -2.16066\end{array}$

13

$(\mathrm{N} 2) \mathrm{Ru}(\mathrm{CH} 3 \mathrm{CN}) \mathrm{Cl} 4 \mathrm{E}(\mathrm{RB} 3 \mathrm{LYP})=-2178.31923645$

$\begin{array}{llll}7 & -0.400122 & 1.003791 & 2.161432\end{array}$

$\begin{array}{llll}7 & 0.010565 & 1.34477 & 3.185861\end{array}$

$\begin{array}{llll}44 & -1.063993 & 0.478793 & 0.488352\end{array}$

$\begin{array}{llll}17 & 1.280067 & 0.195386 & -0.294142\end{array}$

$\begin{array}{llll}17 & -3.36208 & 0.97518 & 1.300094\end{array}$

$\begin{array}{llll}17 & -1.168268 & -1.826739 & 1.310577\end{array}$

$\begin{array}{llll}17 & -1.932507 & -0.162328 & -1.726495\end{array}$

$\begin{array}{llll}6 & -1.016496 & 4.640204 & -1.396282\end{array}$

$\begin{array}{llll}1 & -1.226308 & 5.463006 & -0.697937\end{array}$

$\begin{array}{llll}1 & -0.05306 & 4.843108 & -1.88585\end{array}$

$\begin{array}{llll}6 & -0.992838 & 3.353297 & -0.706606\end{array}$

$\begin{array}{llll}7 & -1.007017 & 2.282558 & -0.227252\end{array}$

$\begin{array}{llll}7 & -1.798309 & 4.641337 & -2.16893\end{array}$

8

(N2)RuCl5 $\quad E(R B 3 L Y P)=-2505.66548118$

$\begin{array}{llll}7 & 1.034123 & 0.069311 & -0.064179\end{array}$

$\begin{array}{llll}7 & 2.198067 & 0.104731 & -0.09987\end{array}$

$\begin{array}{llll}44 & -0.799294 & 0.013334 & -0.008166\end{array}$

$\begin{array}{llll}17 & -0.865586 & 1.806219 & -1.789479\end{array}$

$\begin{array}{llll}17 & -0.756419 & 1.795967 & 1.783302\end{array}$

$\begin{array}{llll}17 & -0.757269 & -1.766218 & -1.802668\end{array}$

$\begin{array}{llll}17 & -0.646157 & -1.776616 & 1.769792\end{array}$

$\begin{array}{llll}17 & -3.332645 & -0.063224 & 0.068787\end{array}$

\begin{tabular}{llll}
8 & & & \\
(N2)RuF3CI2 & \multicolumn{2}{l}{ E(RB3LYP $)=-1424.45609082$} \\
7 & 1.0053 & 0.068258 & -0.063145 \\
7 & 2.185864 & 0.105382 & -0.10056 \\
44 & -0.81755 & 0.012425 & -0.007258 \\
17 & -0.942658 & 1.848826 & -1.83164 \\
17 & -0.717456 & -1.824544 & 1.817737 \\
9 & -0.898461 & 1.469382 & 1.4631 \\
9 & -0.899107 & -1.448162 & -1.47398
\end{tabular}


$\begin{array}{llll}9 & -2.841111 & -0.04806 & 0.053265\end{array}$

13

(N2)RuF4(CH3CN) $\quad E(R B 3 L Y P)=-736.734693824$

$\begin{array}{llll}7 & -0.17913 & 0.542133 & -1.291838\end{array}$

$\begin{array}{llll}7 & 0.642939 & -0.030357 & -0.694343\end{array}$

$\begin{array}{llll}44 & -1.495818 & 1.44986 & -2.239146\end{array}$

$\begin{array}{llll}9 & -0.555009 & 3.218908 & -1.949855\end{array}$

$\begin{array}{llll}9 & -0.468182 & 1.207196 & -3.934995\end{array}$

$\begin{array}{llll}9 & -2.456225 & -0.305996 & -2.672886\end{array}$

$\begin{array}{llll}9 & -2.899081 & 2.420318 & -3.278152\end{array}$

$\begin{array}{llll}6 & -4.563428 & 0.919365 & 0.795502\end{array}$

$\begin{array}{llll}1 & -4.78185 & -0.02611 & 0.26954\end{array}$

$\begin{array}{llll}1 & -5.463148 & 1.551358 & 0.752411\end{array}$

$\begin{array}{llll}1 & -4.372505 & 0.693141 & 1.855515\end{array}$

$\begin{array}{llll}6 & -3.403975 & 1.618012 & 0.176526\end{array}$

$\begin{array}{llll}7 & -2.614856 & 1.595596 & -0.726547\end{array}$

$\begin{array}{llll}8 & & & \\ \text { (N2)RuF4CI } & \text { E(RB3LYP })=-1063.85458959 \\ 7 & -11.820189 & 0.268832 & -0.172721 \\ 7 & -10.834774 & 0.743284 & -0.437293 \\ 44 & -13.71304 & -0.704979 & 0.425529 \\ 9 & -15.217084 & -1.538968 & 0.985236 \\ 17 & -14.916065 & 0.321278 & -1.130297 \\ 9 & -13.697096 & 0.751007 & 1.493341 \\ 9 & -13.050404 & -1.899421 & -0.753731 \\ 9 & -12.795499 & -1.620999 & 1.785308\end{array}$

$\begin{array}{llll}8 & & & \\ \text { (N2)RuF5 } & \text { E(RB3LYP })=-703.625950603 \\ 7 & 1.00691 & 0.068621 & -0.063578 \\ 7 & 2.202199 & 0.106268 & -0.10115 \\ 44 & -0.793028 & 0.013287 & -0.008168 \\ 9 & -0.965318 & 1.494523 & -1.479779 \\ 9 & -0.873896 & 1.485516 & 1.479719 \\ 9 & -0.874763 & -1.464636 & -1.490337 \\ 9 & -0.783044 & -1.472641 & 1.468083 \\ 9 & -2.844239 & -0.047432 & 0.052728\end{array}$

29

$(\mathrm{N} 2) \mathrm{Ru}(\mathrm{NH} 3) 2 \operatorname{mer}(\mathrm{CH} 3 \mathrm{CN}) 3 \quad \mathrm{E}(\mathrm{RB} 3 \mathrm{LYP})=-715.423657113$ 


$\begin{array}{llll}7 & 3.667515 & -1.803869 & 3.397088 \\ 7 & 3.69898 & -2.743755 & 4.03751 \\ 44 & 3.575376 & -0.188667 & 2.278271 \\ 7 & 4.150034 & -1.259872 & 0.657027 \\ 7 & 1.622779 & -0.582522 & 1.864294 \\ 7 & 5.642015 & 0.225597 & 2.721804 \\ 6 & 4.456868 & -1.919475 & -0.260117 \\ 6 & 0.503455 & -0.854759 & 1.662036 \\ 1 & 6.251689 & -0.463103 & 2.266499 \\ 1 & 5.946189 & 1.157557 & 2.419685 \\ 1 & 5.805612 & 0.165226 & 3.733327 \\ 6 & 4.833164 & -2.758181 & -1.395453 \\ 6 & -0.894759 & -1.207039 & 1.425408 \\ 1 & 4.06246 & -2.717898 & -2.17441 \\ 1 & 4.947849 & -3.80071 & -1.074557 \\ 1 & 5.782828 & -2.422195 & -1.828556 \\ 1 & -1.557806 & -0.402505 & 1.764608 \\ 1 & -1.0743 & -1.380004 & 0.357806 \\ 1 & -1.151345 & -2.122127 & 1.972722 \\ 7 & 3.440033 & 1.562616 & 1.048684 \\ 1 & 4.126003 & 1.559886 & 0.285611 \\ 1 & 3.558989 & 2.427062 & 1.588523 \\ 7 & 3.031211 & 0.915471 & 3.887723 \\ 6 & 2.692338 & 1.513937 & 4.834937 \\ 6 & 2.263625 & 2.245121 & 6.024707 \\ 1 & 1.314616 & 2.76144 & 5.837172 \\ 1 & 2.122129 & 1.553141 & 6.86383 \\ 1 & 3.014008 & 2.990781 & 6.313234 \\ 1 & 2.506641 & 1.592206 & 0.622897\end{array}$

\begin{tabular}{|c|c|c|c|}
\hline \multicolumn{4}{|c|}{17} \\
\hline 7 & 1.008038 & 1.024253 & -1.114693 \\
\hline 7 & 1.653174 & 1.660105 & -1.833302 \\
\hline 44 & -0.024814 & -0.007198 & 0.069221 \\
\hline 17 & -1.704674 & -0.305265 & -1.628986 \\
\hline 17 & -1.210477 & -1.311538 & 1.739765 \\
\hline 7 & 0.774635 & -1.86789 & -0.628529 \\
\hline 7 & -1.209209 & 1.656611 & 0.715751 \\
\hline 7 & 1.407232 & 0.177546 & 1.693532 \\
\hline 1 & 0.49016 & -2.567011 & 0.065617 \\
\hline 1 & 1.77617 & -1.928698 & -0.822962 \\
\hline 1 & 0.234472 & -2.009648 & -1.491335 \\
\hline 1 & -1.880823 & 1.739243 & -0.057847 \\
\hline 1 & -1.701656 & 1.335156 & 1.555821 \\
\hline
\end{tabular}




$\begin{array}{llll}1 & -0.746219 & 2.552662 & 0.880844 \\ 1 & 1.544005 & 1.142009 & 2.004699 \\ 1 & 2.319366 & -0.230679 & 1.476454 \\ 1 & 0.930898 & -0.371737 & 2.422245\end{array}$

\begin{tabular}{llll}
28 & \multicolumn{4}{l}{$l$} \\
(N2)RuCl(CH3CN)4 $\mathrm{E}(\mathrm{RB} 3 \mathrm{LYP})=$ & -1195.59521443 \\
7 & -0.71958 & 1.365141 & 1.421592 \\
7 & -0.332324 & 2.035627 & 2.260202 \\
44 & -1.388005 & 0.208275 & -0.025097 \\
17 & -2.190982 & -1.18182 & -1.761903 \\
7 & -0.890531 & 1.561166 & -1.430457 \\
6 & -0.629005 & 2.295951 & -2.297381 \\
6 & -0.312799 & 3.194839 & -3.404267 \\
1 & 0.761445 & 3.410175 & -3.430643 \\
1 & -0.600386 & 2.732095 & -4.355385 \\
1 & -0.854353 & 4.141681 & -3.298142 \\
7 & 0.407461 & -0.675337 & -0.24421 \\
6 & 1.41592 & -1.231406 & -0.427674 \\
6 & 2.664314 & -1.945826 & -0.68133 \\
1 & 2.981137 & -2.496388 & 0.211737 \\
1 & 2.526274 & -2.659688 & -1.501592 \\
1 & 3.460081 & -1.24482 & -0.957717 \\
7 & -3.230707 & 1.010088 & 0.09183 \\
6 & -4.319495 & 1.427563 & 0.102555 \\
6 & -5.69055 & 1.930839 & 0.091921 \\
1 & -5.707041 & 2.997427 & -0.159497 \\
1 & -6.282643 & 1.386807 & -0.652997 \\
1 & -6.157274 & 1.798433 & 1.074626 \\
7 & -1.932671 & -1.225578 & 1.278731 \\
6 & -2.273535 & -2.09755 & 1.973948 \\
6 & -2.710612 & -3.206158 & 2.81843 \\
1 & -1.865847 & -3.616172 & 3.383493 \\
1 & -3.474093 & -2.87085 & 3.529579 \\
1 & -3.136389 & -4.003243 & 2.198072
\end{tabular}

27

$(\mathrm{N} 2) \mathrm{Ru}(\mathrm{NH} 3) 3(\mathrm{CH} 3 \mathrm{CN}) 2 \quad \mathrm{E}(\mathrm{RB} 3 \mathrm{LYP})=-639.232781574$

$\begin{array}{llll}7 & 0.037211 & 0.272152 & -1.841481 \\ 7 & 0.064082 & 0.422297 & -2.971524 \\ 44 & 0.002986 & 0.014029 & 0.094292 \\ 7 & 2.019829 & -0.170513 & 0.124637 \\ 7 & -0.195304 & -2.121543 & -0.201731 \\ 1 & 0.723787 & -2.574528 & -0.257354\end{array}$




$\begin{array}{llll}1 & -0.734497 & -2.583158 & 0.539164 \\ 1 & -0.682468 & -2.305477 & -1.086378 \\ 6 & -4.633315 & 0.445615 & -0.017593 \\ 1 & -5.12892 & -0.47553 & 0.31187 \\ 1 & -4.975547 & 1.270799 & 0.618492 \\ 1 & -4.940669 & 0.653808 & -1.049847 \\ 6 & -3.180983 & 0.304714 & 0.05723 \\ 7 & -2.014957 & 0.193616 & 0.100811 \\ 7 & -0.018167 & -0.272322 & 2.23239 \\ 1 & -0.976159 & -0.268116 & 2.601223 \\ 1 & 0.506243 & 0.464049 & 2.718891 \\ 7 & 0.18426 & 2.152392 & 0.377056 \\ 1 & 0.415958 & -1.163895 & 2.497973 \\ 1 & -0.348019 & 2.494801 & 1.184637 \\ 1 & -0.174775 & 2.644824 & -0.449073 \\ 1 & 1.16439 & 2.431452 & 0.497186 \\ 6 & 3.187438 & -0.27184 & 0.098168 \\ 6 & 4.642375 & -0.395245 & 0.044678 \\ 1 & 5.120545 & 0.425302 & 0.593136 \\ 1 & 4.967113 & -1.344035 & 0.488558 \\ 1 & 4.987029 & -0.363223 & -0.996228\end{array}$

31

$(\mathrm{N} 2) \mathrm{Ru}(\mathrm{NH} 3)(\mathrm{CH} 3 \mathrm{CN}) 4 \quad \mathrm{E}(\mathrm{RB} 3 \mathrm{LYP})=-791.613657671$

$\begin{array}{llll}7 & -2.2151 & 0.129593 & 0.797852 \\ 7 & -1.594463 & 0.297358 & 1.733907 \\ 44 & -3.295791 & -0.164943 & -0.834875 \\ 7 & -3.710816 & -2.056488 & -0.237393 \\ 6 & -3.929511 & -3.140008 & 0.142655 \\ 6 & -4.193104 & -4.490236 & 0.633314 \\ 1 & -5.271242 & -4.657978 & 0.740316 \\ 1 & -3.791624 & -5.23806 & -0.060633 \\ 1 & -3.72026 & -4.636078 & 1.612025 \\ 7 & -2.893934 & 1.723726 & -1.450269 \\ 6 & -2.640069 & 2.819972 & -1.76706 \\ 6 & -2.313422 & 4.19217 & -2.146208 \\ 1 & -3.205102 & 4.714331 & -2.512618 \\ 1 & -1.920936 & 4.740302 & -1.281221 \\ 1 & -1.55393 & 4.202677 & -2.936796 \\ 7 & -4.949366 & 0.501564 & 0.128559 \\ 6 & -5.877941 & 0.894866 & 0.719846 \\ 6 & -7.026896 & 1.389394 & 1.473951 \\ 1 & -6.712485 & 1.71139 & 2.474051 \\ 1 & -7.485264 & 2.243927 & 0.962404 \\ 1 & -7.782884 & 0.603113 & 1.584631\end{array}$




$\begin{array}{llll}6 & 0.538537 & -1.675887 & -2.998964 \\ 1 & 1.415399 & -1.38943 & -2.405882 \\ 1 & 0.529269 & -2.767668 & -3.098484 \\ 1 & 0.636341 & -1.235965 & -3.99842 \\ 6 & -0.683659 & -1.20972 & -2.3491 \\ 7 & -1.653869 & -0.833314 & -1.816847 \\ 7 & -4.45585 & -0.490419 & -2.594089 \\ 1 & -5.458206 & -0.468416 & -2.378104 \\ 1 & -4.238445 & -1.402881 & -3.008633 \\ 1 & -4.265247 & 0.232671 & -3.296007\end{array}$

\begin{tabular}{llll}
28 & & & \\
(N2)RuF(CH3CN)4 & \multicolumn{2}{l}{$\mathrm{E}(\mathrm{RB} 3 \mathrm{LYP})=-835.212796354$} \\
7 & -0.858861 & 0.732758 & 2.612221 \\
7 & -0.651285 & 0.896781 & 3.724567 \\
44 & -1.212699 & 0.452622 & 0.71271 \\
9 & -1.56853 & 0.169944 & -1.198916 \\
7 & -2.03126 & -1.355148 & 1.034509 \\
6 & -2.519344 & -2.40981 & 1.132641 \\
6 & -3.13346 & -3.731186 & 1.233957 \\
1 & -2.440504 & -4.443111 & 1.69663 \\
1 & -4.043376 & -3.686681 & 1.843194 \\
1 & -3.398583 & -4.099288 & 0.236166 \\
7 & -0.427716 & 2.233823 & 0.209665 \\
6 & 0.009566 & 3.247921 & -0.165259 \\
6 & 0.553204 & 4.512422 & -0.654075 \\
1 & -0.017126 & 5.357346 & -0.251692 \\
1 & 1.600162 & 4.624239 & -0.349966 \\
1 & 0.501287 & 4.545378 & -1.748413 \\
7 & 0.575555 & -0.415297 & 0.412601 \\
6 & 1.590207 & -0.928676 & 0.153582 \\
6 & 2.854558 & -1.574001 & -0.190533 \\
1 & 2.877789 & -1.807223 & -1.26125 \\
1 & 3.698774 & -0.915048 & 0.042223 \\
1 & 2.97628 & -2.506093 & 0.372913 \\
7 & -3.034672 & 1.29372 & .831342 \\
6 & -4.100855 & 1.766043 & 0.813183 \\
6 & -5.437075 & 2.354314 & 0.769797 \\
1 & -6.12312 & 1.799014 & 1.41946 \\
1 & -5.40929 & 3.397283 & 1.105269 \\
1 & -5.826307 & 2.326625 & -0.254465 \\
& & &
\end{tabular}




\begin{tabular}{llll}
20 & & & \\
(N2)RuCl(NH3)4 & \multicolumn{2}{l}{$\mathrm{E}(\mathrm{RB} 3 \mathrm{LYP})=$} & -890.873152649 \\
7 & 0.151321 & 0.634637 & 2.071543 \\
7 & 0.189616 & 0.157264 & 3.119392 \\
44 & 0.086508 & 1.425023 & 0.336648 \\
17 & 0.002092 & 2.413678 & -1.832415 \\
7 & -0.766137 & -0.333057 & -0.579213 \\
1 & -1.685709 & -0.589785 & -0.206583 \\
1 & -0.170743 & -1.164344 & -0.510258 \\
1 & -0.866862 & -0.07336 & -1.569296 \\
7 & 0.930871 & 3.293002 & 1.00937 \\
1 & 1.876385 & 3.203625 & 1.394297 \\
1 & 0.36307 & 3.773353 & 1.714464 \\
1 & 0.968193 & 3.869875 & 0.158717 \\
7 & 2.060181 & 0.729928 & -0.193775 \\
1 & 2.150275 & -0.29101 & -0.191151 \\
1 & 2.802531 & 1.09883 & .408947 \\
1 & 2.204039 & 1.076189 & -1.151488 \\
7 & -1.895391 & 2.227676 & 0.630512 \\
1 & -2.609298 & 1.512573 & 0.801384 \\
1 & -1.957156 & 2.903852 & 1.398195 \\
1 & -2.105803 & 2.706787 & -0.255037
\end{tabular}

\begin{tabular}{llll}
20 & & & \\
(N2)RuF(NH3)4 & \multicolumn{2}{l}{$E($ RB3LYP $)=-530.494016793$} \\
7 & 0.15169 & 0.614981 & 2.133366 \\
7 & 0.191771 & 0.138613 & 3.183108 \\
44 & 0.087367 & 1.393861 & 0.404553 \\
9 & 0.024067 & 2.201051 & -1.423727 \\
7 & -0.760076 & -0.308666 & -0.587772 \\
1 & -1.692708 & -0.579033 & -0.261361 \\
1 & -0.177076 & -1.150715 & -0.567275 \\
1 & -0.822306 & 0.043356 & -1.552999 \\
7 & 0.925617 & 3.283714 & 0.983056 \\
1 & 1.872111 & 3.227395 & 1.370945 \\
1 & 0.357937 & 3.815981 & 1.64939 \\
1 & 0.952754 & 3.782632 & 0.083518 \\
7 & 2.040354 & 0.747708 & -0.210594 \\
1 & 2.159268 & -0.268598 & -0.261671 \\
1 & 2.811879 & 1.113882 & 0.355301 \\
1 & 2.090386 & 1.14619 & -1.158261 \\
7 & -1.880574 & 2.227509 & 0.6103 \\
1 & -2.62351 & 1.535003 & 0.744772 \\
1 & -1.974742 & 2.919611 & 1.359814 \\
1 & -2.006224 & 2.690264 & -0.300207
\end{tabular}




\begin{tabular}{|c|c|c|c|}
\hline \multicolumn{4}{|c|}{ Cis-Carbonyl Species } \\
\hline \multicolumn{4}{|c|}{$\mathrm{cCO}$ is cis oriented to $\mathrm{N}_{2}$ group } \\
\hline \multicolumn{4}{|c|}{25} \\
\hline 7 & 1.799241 & 0.204736 & 1.395088 \\
\hline 7 & 2.666661 & 0.103019 & 2.112803 \\
\hline 44 & 0.238547 & 0.354477 & 0.109584 \\
\hline 6 & -0.471578 & 1.881637 & 1.055872 \\
\hline 8 & -0.91332 & 2.790381 & 1.621944 \\
\hline 7 & -0.840946 & -0.912274 & 1.356381 \\
\hline 6 & -1.441643 & -1.608541 & 2.07637 \\
\hline 6 & -2.187023 & -2.469458 & 2.986785 \\
\hline 1 & -3.24684 & -2.496872 & 2.705709-- \\
\hline 1 & -1.790104 & -3.491479 & 2.959288 \\
\hline 1 & -2.108799 & -2.092015 & 4.013955 \\
\hline 7 & 0.973677 & -1.311373 & -0.894799 \\
\hline 6 & 1.40886 & -2.234756 & -1.462238 \\
\hline 6 & 1.965504 & -3.380906 & -2.170852 \\
\hline 1 & 2.49892 & -3.048591 & -3.070023 \\
\hline 1 & 2.671378 & -3.920118 & -1.527122 \\
\hline 1 & 1.168088 & -4.071219 & -2.471381 \\
\hline 6 & -3.359051 & 0.566546 & -2.828794 \\
\hline 1 & -3.506556 & 1.606488 & -3.146111 \\
\hline 1 & -3.173327 & -0.046586 & -3.719036 \\
\hline 6 & -2.234089 & 0.478486 & -1.905774 \\
\hline 7 & -1.331957 & 0.421254 & -1.168167 \\
\hline 1 & -4.27929 & 0.215557 & -2.346157 \\
\hline 6 & 1.246016 & 1.503016 & -1.072416 \\
\hline 8 & 1.844463 & 2.182225 & -1.79489 \\
\hline
\end{tabular}

\begin{tabular}{lllll}
10 & & \multicolumn{2}{c}{$E(\mathrm{RB} 3 \mathrm{LYP})=-1812.03330077$} & $\mathrm{x}$ \\
$(\mathrm{N} 2) \mathrm{Ru}(\mathrm{cCO}) 2 \mathrm{Cl} 3$ & \multicolumn{2}{c}{0.857933} \\
7 & -0.286893 & 1.829339 & 0.313136 \\
7 & -0.568698 & 2.831904 & 1.3135 \\
44 & 0.139355 & 0.062767 & 0.05042 \\
6 & 1.499808 & 0.690923 & -1.081954 \\
8 & 2.353136 & 1.071502 & -1.799742 \\
17 & 0.474234 & -2.082264 & -0.944693 \\
17 & -1.399247 & 0.72887 & -1.724141 \\
17 & -1.674644 & -0.786766 & 1.44624 \\
6 & 1.28098 & -0.508416 & 1.427959 \\
8 & 1.996577 & -0.881061 & 2.286597
\end{tabular}




\begin{tabular}{llll}
19 & & & \\
(N2)Ru(cCO)2(NH3)3 & \multicolumn{2}{c}{$\mathrm{E}(\mathrm{RB} 3 \mathrm{LYP})=-6$} \\
7 & 0.865337 & 0.890469 & -1.092845 \\
7 & 1.974391 & 0.932456 & -1.318682 \\
44 & -1.122069 & 0.755654 & -0.688188 \\
6 & -0.859844 & -0.936019 & 0.199799 \\
6 & -1.431916 & -0.160999 & -2.356234 \\
8 & -1.614986 & -0.708564 & -3.363039 \\
8 & -0.702177 & -1.955862 & 0.731022 \\
7 & -3.219826 & 0.680625 & -0.210923 \\
1 & -3.382098 & 0.649802 & 0.803952 \\
1 & -3.745673 & 1.483868 & -0.577894 \\
1 & -3.663711 & -0.162283 & -0.598797 \\
7 & -1.419211 & 2.693736 & -1.701031 \\
1 & -1.528489 & 3.488498 & -1.058533 \\
1 & -0.612118 & 2.909942 & -2.301607 \\
1 & -2.239871 & 2.691178 & -2.320535 \\
7 & -0.752024 & 1.855054 & 1.191306 \\
1 & 0.070859 & 2.466071 & 1.104087 \\
1 & -1.535849 & 2.450823 & 1.486781 \\
1 & -0.553157 & 1.218306 & 1.973809
\end{tabular}

10

\begin{tabular}{lrlll}
$(\mathrm{N} 2) \mathrm{Ru}(\mathrm{cCO}) 2 \mathrm{~F} 3$ & \multicolumn{2}{c}{$\mathrm{E}(\mathrm{RB} 3 \mathrm{RYP})=-730.874145539$} & $\mathrm{x}$ \\
7 & -0.320325 & -0.898098 & -1.597432 &
\end{tabular}

$\begin{array}{llll}7 & -0.535263 & -1.459428 & -2.565062\end{array}$

$\begin{array}{llll}44 & -0.071277 & -0.014174 & 0.146156\end{array}$

$\begin{array}{llll}6 & 1.792538 & -0.214266 & 0.18016\end{array}$

$\begin{array}{llll}8 & 2.967356 & -0.352814 & 0.221182\end{array}$

$\begin{array}{llll}9 & -0.027763 & 0.709164 & 1.9721\end{array}$

$\begin{array}{llll}9 & -0.260534 & -1.835241 & 0.928724\end{array}$

$\begin{array}{llll}9 & -2.058856 & 0.108792 & 0.19761\end{array}$

$\begin{array}{llll}6 & 0.004634 & 1.726087 & -0.548242\end{array}$

$\begin{array}{llll}8 & 0.036856 & 2.829976 & -0.974228\end{array}$

$\begin{array}{llll}17 & & & \\ (\mathrm{~N} 2) \mathrm{Ru}(\mathrm{cCO}) 3(\mathrm{NH} 3) 2 & \mathrm{E}(\mathrm{RB} 3 \mathrm{LYP})=-656.983203317 & \mathrm{x} \\ 7 & 0.038253 & 0.031438 & -1.209968 \\ 7 & 0.068208 & 0.039758 & -2.340514 \\ 44 & 0.030673 & -0.008724 & 0.843969 \\ 7 & -0.014274 & -0.030916 & 2.999378 \\ 1 & 0.014131 & -0.987724 & 3.375959 \\ 1 & 0.788856 & 0.465503 & 3.40752 \\ 1 & -0.861487 & 0.412361 & 3.377572\end{array}$




$\begin{array}{llll}6 & 1.74293 & -0.945294 & 0.859912 \\ 6 & 0.983702 & 1.749634 & 0.875082 \\ 6 & -0.935295 & -1.759937 & 0.800194 \\ 8 & -1.486662 & -2.773413 & 0.76313 \\ 8 & 2.757608 & -1.500196 & 0.864387 \\ 8 & 1.539448 & 2.761389 & 0.881028 \\ 7 & -1.90417 & 1.049172 & 0.816637 \\ 1 & -2.210087 & 1.210358 & -0.152826 \\ 1 & -1.864645 & 1.973656 & 1.265054 \\ 1 & -2.659505 & 0.522337 & 1.274095\end{array}$

\begin{tabular}{|c|c|c|c|}
\hline & $\mathrm{u}(\mathrm{cCO}) 3(\mathrm{Ac}$ & c) $\quad E(F$ & $3 L Y P)=-889.408236539$ \\
\hline 7 & 2.010377 & -1.59136 & 0.366182 \\
\hline 7 & 3.049155 & -2.016771 & 0.508008 \\
\hline 44 & 0.191459 & -0.712184 & 0.106971 \\
\hline 6 & -0.78178 & -2.383385 & 0.095516 \\
\hline 6 & 0.381901 & -0.753296 & -1.872586 \\
\hline 6 & -0.065044 & -0.517455 & 2.069278 \\
\hline 8 & -0.225967 & -0.378112 & 3.206665 \\
\hline 8 & 0.473157 & -0.752946 & -3.026071 \\
\hline 8 & -1.409388 & -3.362172 & 0.083767 \\
\hline 6 & 0.673026 & 2.280775 & -0.004257 \\
\hline 6 & -1.736066 & 1.578133 & -0.240333 \\
\hline 6 & 1.677457 & 3.402508 & 0.043754 \\
\hline 1 & 1.199553 & 4.381452 & -0.031159 \\
\hline 1 & 2.250306 & 3.351011 & 0.977927 \\
\hline 1 & 2.397662 & 3.291954 & -0.77703 \\
\hline 6 & -3.175007 & 1.986351 & -0.427836 \\
\hline 1 & -3.569169 & 1.548315 & -1.353577 \\
\hline 1 & -3.28634 & 3.07184 & -0.47093 \\
\hline 1 & -3.785069 & 1.593439 & 0.394921 \\
\hline 8 & 1.196648 & 1.073478 & 0.11766 \\
\hline 8 & -1.569847 & 0.263129 & -0.156116 \\
\hline 6 & -0.706584 & 2.538 & -0.17022 \\
\hline 1 & -1.002739 & 3.575347 & -0.255245 \\
\hline
\end{tabular}

21

$(\mathrm{N} 2) \mathrm{Ru}(\mathrm{cCO}) 3(\mathrm{CH} 3 \mathrm{CN}) 2 \mathrm{E}(\mathrm{RB} 3 \mathrm{LYP})=-809.370391828$ not in list- is in plot ?

$\begin{array}{llll}7 & 1.135645 & -2.004975 & 0.224104 \\ 7 & 1.826297 & -2.895098 & 0.312218 \\ 44 & -0.094702 & -0.385129 & 0.064113 \\ 6 & -1.682783 & -1.508697 & 0.165501 \\ 6 & -0.079189 & -0.535929 & -1.934843\end{array}$




$\begin{array}{llll}6 & -0.094309 & -0.152908 & 2.055356 \\ 8 & -0.091283 & 0.003287 & 3.197384 \\ 8 & -0.069081 & -0.599486 & -3.085704 \\ 8 & -2.631498 & -2.166083 & 0.22482 \\ 7 & -1.272832 & 1.260966 & -0.09785 \\ 7 & 1.594062 & 0.818501 & -0.044515 \\ 6 & 2.553237 & 1.483312 & -0.104943 \\ 6 & -1.960471 & 2.199925 & -0.19025 \\ 6 & 3.754822 & 2.303518 & -0.17917 \\ 1 & 3.67019 & 3.033439 & -0.994037 \\ 1 & 3.907095 & 2.844376 & 0.763352 \\ 1 & 4.633676 & 1.673085 & -0.365756 \\ 6 & -2.827574 & 3.364785 & -0.30465 \\ 1 & -3.864037 & 3.049498 & -0.481403 \\ 1 & -2.508364 & 3.999346 & -1.141017 \\ 1 & -2.79559 & 3.95712 & 0.6186\end{array}$

11

(N2)Ru(cCO)3Cl2

$7 \quad 0.850587$

$E($ RB3LYP $)=-1464.97050965 x$

$\begin{array}{llll}7 & 1.967088 & 1.297496 & -0.865233\end{array}$

$\begin{array}{llll}44 & -1.149423 & 1.013901 & -0.450951\end{array}$

$\begin{array}{llll}17 & -3.50916 & 0.764585 & -0.283676\end{array}$

$\begin{array}{llll}17 & -1.216876 & 0.511357 & -2.805729\end{array}$

$\begin{array}{llll}6 & -1.151893 & 1.407479 & 1.417052\end{array}$

$\begin{array}{llll}8 & -1.180258 & 1.64631 & 2.562283\end{array}$

$\begin{array}{llll}6 & -1.079092 & -0.937945 & -0.195035\end{array}$

$\begin{array}{llll}8 & -1.104486 & -2.094279 & -0.09571\end{array}$

$\begin{array}{llll}6 & -1.533842 & 2.867154 & -0.996573\end{array}$

$\begin{array}{llll}8 & -1.823973 & 3.929277 & -1.364481\end{array}$

\begin{tabular}{|c|c|c|c|}
\hline \multicolumn{2}{|c|}{$\begin{array}{l}11 \\
(\mathrm{~N} 2) \mathrm{Ru}(\mathrm{cCO}) 3 \mathrm{~F} 2\end{array}$} & \multicolumn{2}{|c|}{$E(R B 3 L Y P)=-744.197334038$} \\
\hline 7 & 0.79438 & 1.18637 & -0.73841 \\
\hline 7 & 1.905129 & 1.27537 & -0.953042 \\
\hline 44 & -1.188672 & 1.002444 & -0.485027 \\
\hline 9 & -3.14414 & 0.779998 & -0.443574 \\
\hline 9 & -1.248595 & 0.587056 & -2.428442 \\
\hline 6 & -1.189081 & 1.390129 & 1.379666 \\
\hline 8 & -1.21186 & 1.624623 & 2.528304 \\
\hline 6 & -1.105203 & -0.951477 & -0.204671 \\
\hline 8 & -1.130177 & -2.105488 & -0.093678 \\
\hline 6 & -1.561021 & 2.874388 & -0.994683 \\
\hline 8 & -1.852088 & 3.943148 & -1.338016 \\
\hline
\end{tabular}




$\begin{aligned} & 17 \\ & \text { (N2)Ru(cCO)3(NH3)2 }\end{aligned}$
$\begin{array}{lllll} & E(\mathrm{RB} 3 \mathrm{LYP})=-656.983172628 & \mathrm{x} \text { in twice ? } \\ 7 & 0.866767 & 0.930083 & -1.056033 & \\ 7 & 1.975062 & 0.977068 & -1.276387 & \\ 44 & -1.13917 & 0.799422 & -0.631903 & \\ 6 & -0.839408 & -0.813066 & 0.426148 & \\ 6 & -1.403486 & -0.315274 & -2.271808 & \\ 6 & -0.863795 & 1.928285 & 0.996215 & \\ 8 & -0.693035 & 2.579428 & 1.933963 & \\ 8 & -1.543344 & -0.959093 & -3.219678 & \\ 8 & -0.65707 & -1.768347 & 1.051991 & \\ 7 & -3.251962 & 0.694428 & -0.214371 & \\ 1 & -3.665255 & -0.190094 & -0.538447 & \\ 1 & -3.450951 & 0.75591 & 0.793005 & \\ 1 & -3.777825 & 1.452607 & -0.668702 & \\ 7 & -1.471483 & 2.622171 & -1.827558 & \\ 1 & -1.883016 & 3.392467 & -1.284619 & \\ 1 & -0.578399 & 2.974811 & -2.19858 & \\ 1 & -2.080562 & 2.465361 & -2.641107\end{array}$

\begin{tabular}{|c|c|c|c|}
\hline \\
\hline 7 & 0.793393 & 0.991123 & -0.959278 \\
\hline 7 & 1.887385 & 1.004977 & -1.241922 \\
\hline 44 & -1.194122 & 0.965844 & -0.445879 \\
\hline 6 & -1.51624 & 2.650547 & -1.503002 \\
\hline 6 & -0.922231 & -0.719146 & 0.624913 \\
\hline 6 & -1.626704 & -0.138868 & -2.074525 \\
\hline 6 & -0.81079 & 2.069911 & 1.19543 \\
\hline 8 & -0.606644 & 2.699774 & 2.136809 \\
\hline 8 & -1.715713 & 3.612548 & -2.102502 \\
\hline 8 & -1.888566 & -0.769629 & -3.000916 \\
\hline 8 & -0.782132 & -1.681168 & 1.240912 \\
\hline 6 & -5.706086 & 0.905807 & 0.716442 \\
\hline 1 & -5.828076 & 1.125666 & 1.785274 \\
\hline 1 & -6.139396 & -0.081807 & 0.510287 \\
\hline 1 & -6.258995 & 1.658302 & 0.138771 \\
\hline 6 & -4.296812 & 0.925097 & 0.353881 \\
\hline 7 & -3.165206 & 0.940356 & 0.06274 \\
\hline
\end{tabular}




\begin{tabular}{lllll}
12 & \multicolumn{3}{c}{$E(\mathrm{RB} 3 L Y P)=-1117.74998195$} & $x$ \\
(N2)Ru(cCO)4Cl & \multicolumn{2}{l}{$\mathrm{R}$} \\
7 & 0.97867 & 1.006691 & -1.063603 & \\
7 & 2.079917 & 1.046317 & -1.320899 \\
44 & -1.027987 & 0.933952 & -0.595004 \\
17 & -3.335931 & 0.848667 & -0.056338 \\
6 & -1.466737 & 2.588307 & -1.618873 \\
6 & -0.85319 & -0.730088 & 0.489992 \\
6 & -1.500093 & -0.166774 & -2.189651 \\
6 & -0.820834 & 2.025126 & 1.061462 \\
8 & -0.759887 & 2.651842 & 2.029407 \\
8 & -1.777886 & 3.539281 & -2.195268 \\
8 & -1.830815 & -0.803125 & -3.09468 \\
8 & -0.8108 & -1.691524 & 1.128157
\end{tabular}

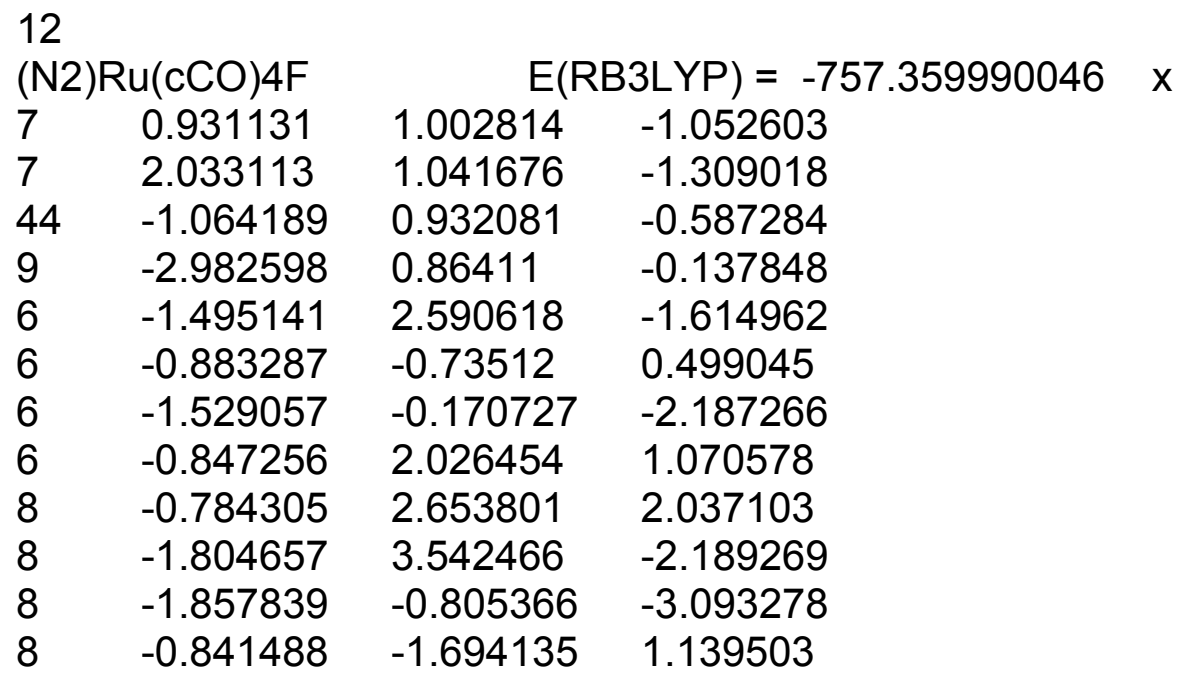

\begin{tabular}{|c|c|c|c|}
\hline ( & $\mathrm{u}(\mathrm{cCO}) 4(\mathrm{Nr}$ & $E(R$ & $3 \mathrm{LYP})=-713.677352921$ \\
\hline 7 & 0.837336 & 1.001637 & -1.031218 \\
\hline 7 & 1.936323 & 1.042204 & -1.290161 \\
\hline 44 & -1.177178 & 0.925122 & -0.557505 \\
\hline 6 & -1.483033 & 2.613407 & -1.611271 \\
\hline 6 & -0.859951 & -0.756363 & 0.503937 \\
\hline 6 & -1.525113 & -0.17167 & -2.209878 \\
\hline 6 & -0.822567 & 2.028098 & 1.089161 \\
\hline 8 & -0.609614 & 2.659922 & 2.02819 \\
\hline 8 & -1.64714 & 3.579298 & -2.216567 \\
\hline 8 & -1.713898 & -0.798566 & -3.157289 \\
\hline 8 & -0.667451 & -1.717873 & 1.107772 \\
\hline r & -3.282647 & 0.829317 & -0.073454 \\
\hline
\end{tabular}




$\begin{array}{llll}1 & -3.633107 & -0.138209 & -0.070116 \\ 1 & -3.488557 & 1.221072 & 0.855265 \\ 1 & -3.859768 & 1.349231 & -0.748284\end{array}$

\begin{tabular}{llll}
21 & \multicolumn{4}{l}{$l$} & \\
(N2)Ru(cCO)(NH3)4 & & E(RB3LYP $)=-543.567168895$ & $x$ \\
7 & -1.031765 & 0.306152 & 1.370296 \\
7 & -1.499944 & -0.554517 & 1.947029 \\
44 & -0.213365 & 1.804636 & 0.353046 \\
6 & 1.021101 & 0.631698 & -0.464384 \\
8 & 1.787045 & -0.094097 & -0.966355 \\
7 & -1.662988 & 3.165003 & 1.354582 \\
1 & -2.617517 & 3.051586 & 0.990667 \\
1 & -1.719054 & 2.950427 & 2.358753 \\
1 & -1.432032 & 4.16319 & 1.276082 \\
7 & 1.180954 & 2.160085 & 1.989299 \\
1 & 0.939127 & 2.967605 & 2.576375 \\
1 & 2.1408 & 2.304973 & 1.652074 \\
1 & 1.216072 & 1.3395 & 2.607783 \\
7 & -1.626469 & 1.475711 & -1.27178 \\
1 & -2.026792 & 0.530936 & -1.205853 \\
1 & -2.415317 & 2.13347 & -1.286072 \\
1 & -1.171934 & 1.532142 & -2.191364 \\
1 & 1.265782 & 4.039856 & -0.157873 \\
1 & 1.124158 & 3.197571 & -1.562526 \\
1 & -0.122855 & 4.144073 & -1.043938 \\
7 & 0.605873 & 3.492987 & -0.725527
\end{tabular}

33

(N2)Ru(cCO)(Acac)2 $\quad E(R B 3 L Y P)=-1008.22131311$ not in list

$\begin{array}{llll}7 & -1.057674 & 1.330165 & 2.043277\end{array}$

$\begin{array}{llll}7 & -1.822685 & 1.005832 & 2.824291\end{array}$

$\begin{array}{llll}44 & 0.296716 & 1.891333 & 0.762106\end{array}$

$\begin{array}{llll}6 & -0.243478 & 0.638666 & -0.504702\end{array}$

$8 \quad-0.582491 \quad-0.135306 \quad-1.328805$

$\begin{array}{llll}8 & 1.727725 & 2.621912 & -0.479527\end{array}$

$\begin{array}{llll}8 & 1.640404 & 0.600166 & 1.622638\end{array}$

$\begin{array}{llll}6 & 2.987436 & 2.25748 & -0.500407\end{array}$

$\begin{array}{llll}6 & 3.829411 & 2.995673 & -1.520405\end{array}$

$\begin{array}{llll}1 & 4.87492 & 2.676427 & -1.498115\end{array}$

$\begin{array}{llll}1 & 3.421577 & 2.826233 & -2.524811\end{array}$

$\begin{array}{llll}1 & 3.775938 & 4.074493 & -1.328297\end{array}$

$\begin{array}{llll}6 & 3.572021 & 1.274679 & 0.327299\end{array}$

$\begin{array}{llll}6 & 2.910822 & 0.522841 & 1.329771\end{array}$ 


$\begin{array}{llll}6 & 3.686346 & -0.472436 & 2.168761 \\ 1 & 4.739056 & -0.523758 & 1.877669 \\ 1 & 3.234683 & -1.467447 & 2.071969 \\ 1 & 4.630772 & 1.088178 & 0.193952 \\ 1 & 3.617824 & -0.187573 & 3.226118 \\ 8 & -0.964702 & 3.31112 & -0.03225 \\ 8 & 0.984439 & 3.292364 & 2.12573 \\ 6 & -1.011076 & 4.576847 & 0.292102 \\ 6 & -1.970132 & 5.397336 & -0.547716 \\ 1 & -2.014108 & 6.440172 & -0.221559 \\ 1 & -1.6542 & 5.361158 & -1.59795 \\ 1 & -2.974111 & 4.958455 & -0.497272 \\ 6 & -0.267891 & 5.197113 & 1.32533 \\ 6 & 0.663166 & 4.559875 & 2.176544 \\ 6 & 1.376482 & 5.353003 & 3.253056 \\ 1 & 1.054068 & 6.397677 & 3.27815 \\ 1 & 2.45937 & 5.31363 & 3.079092 \\ 1 & -0.429502 & 6.257563 & 1.476913 \\ 1 & 1.190616 & 4.892809 & 4.231456\end{array}$

\begin{tabular}{lllll}
12 & \multicolumn{3}{c}{$E(\mathrm{RB} 3 \mathrm{LYP})=-1755.28655692$} & $\mathrm{x}$ \\
$(\mathrm{N} 2) \mathrm{Ru}(\mathrm{cCO}) \mathrm{aCl} 3$ & \multicolumn{2}{c}{-0.323111} & \\
7 & 0.006369 & -1.884676 & -0.530021 \\
7 & 0.009486 & -3.010547 & -0.004731 \\
44 & -0.010473 & 0.016305 & 0.0047 \\
6 & -0.082359 & -0.154523 & 1.829005 \\
8 & -0.113573 & -0.265836 & 3.012576 \\
17 & 0.091565 & 2.40535 & 0.32689 \\
17 & -2.417164 & -0.040024 & -0.088322 \\
17 & 0.271749 & 0.249073 & -2.466468 \\
7 & 2.129478 & 0.248482 & -0.126648 \\
1 & 2.70692 & -0.422918 & 0.37874 \\
1 & 2.264511 & 1.210138 & 0.205283 \\
1 & 2.257534 & 0.198415 & -1.146398
\end{tabular}

$\begin{array}{llll}23 & & & \\ \text { (N2)Ru(cCO)(CH3CN)-(NH3)3 } & \mathrm{E}(\mathrm{RB} 3 \mathrm{LYP})=-619.764243226 & \mathrm{x} \\ 7 & 1.829644 & 0.20016 & -0.545759 \\ 7 & 2.962362 & 0.259155 & -0.592508 \\ 44 & -0.158998 & 0.071345 & -0.412808 \\ 6 & -0.0742 & -1.822362 & -0.392631 \\ 8 & -0.021936 & -2.987585 & -0.363188 \\ 7 & -0.298023 & 0.089784 & -2.57286 \\ 1 & -0.183517 & 1.029181 & -2.972417\end{array}$




$\begin{array}{llll}1 & 0.441163 & -0.496581 & -2.980919 \\ 7 & -0.257354 & 2.282133 & -0.380545 \\ 1 & 0.647367 & 2.698656 & -0.634113 \\ 1 & -0.962329 & 2.699005 & -1.00043 \\ 7 & -2.299624 & -0.012927 & -0.225995 \\ 1 & -2.765567 & 0.883193 & -0.411754 \\ 1 & -2.731255 & -0.713571 & -0.840967 \\ 1 & -2.535121 & -0.282109 & 0.737517 \\ 1 & -0.466646 & 2.593568 & 0.575808 \\ 1 & -1.189777 & -0.281145 & -2.922371 \\ 7 & -0.02688 & 0.121475 & 1.631325 \\ 6 & 0.115817 & 0.088775 & 2.792908 \\ 6 & 0.31218 & 0.030001 & 4.237896 \\ 1 & -0.567034 & 0.417835 & 4.766586 \\ 1 & 0.479345 & -1.007681 & 4.553173 \\ 1 & 1.18611 & 0.625992 & 4.528866\end{array}$

\begin{tabular}{llll}
29 & \multicolumn{4}{l}{} \\
(N2) $\mathrm{Ru}(\mathrm{CCO})(\mathrm{CH} 3 \mathrm{CN}) 4$ & $\mathrm{E}(\mathrm{RB} 3 \mathrm{LYP})=-848$ \\
7 & 0.482722 & -2.143976 & -0.070229 \\
7 & 0.67185 & -3.254021 & 0.04747 \\
44 & 0.145827 & -0.176894 & -0.256616 \\
6 & 0.309438 & -0.270707 & -2.152751 \\
8 & 0.406685 & -0.314201 & -3.310675 \\
7 & -0.036556 & -0.059003 & 1.819996 \\
6 & -0.135111 & -0.007963 & 2.982437 \\
6 & -0.256852 & 0.046349 & 4.435628 \\
1 & -0.023967 & -0.931895 & 4.873144 \\
1 & 0.436286 & 0.787661 & 4.850503 \\
1 & -1.277872 & 0.322786 & 4.724103 \\
7 & -0.198774 & 1.811995 & -0.387144 \\
6 & -0.395712 & 2.958198 & -0.477546 \\
6 & -0.640413 & 4.391121 & -0.603647 \\
1 & -1.636488 & 4.645028 & -0.222089 \\
1 & 0.106349 & 4.959012 & -0.036099 \\
1 & -0.582689 & 4.69407 & -1.656174 \\
7 & 2.142169 & 0.1757 & -0.07867 \\
6 & 3.291067 & 0.361645 & 0.007436 \\
6 & 4.730592 & 0.580421 & 0.102106 \\
1 & 4.949131 & 1.425441 & 0.76558 \\
1 & 5.226134 & -0.313584 & 0.499499 \\
1 & 5.147452 & 0.796743 & -0.888977 \\
7 & -1.852818 & -0.536841 & -0.388431 \\
6 & -2.99476 & -0.759751 & -0.47912 \\
6 & -4.418931 & -1.050381 & -0.607084
\end{tabular}




$\begin{array}{llll}1 & -5.008487 & -0.372731 & 0.021583 \\ 1 & -4.738822 & -0.928324 & -1.648997 \\ 1 & -4.626038 & -2.082295 & -0.298961\end{array}$

\begin{tabular}{llll}
19 & \multicolumn{3}{l}{$l$} \\
(N2)Ru(cCO)Cl2(CH3CN)2 & $\mathrm{E}(\mathrm{RB} 3 \mathrm{LYP})=-1503.85397232$ & $\mathrm{x}$ \\
7 & -1.659506 & 1.440074 & 0.00723 \\
7 & -2.489142 & 2.193484 & 0.187521 \\
44 & -0.261457 & 0.080426 & -0.231991 \\
6 & -0.346969 & 0.158714 & -2.102011 \\
8 & -0.398699 & 0.193088 & -3.279676 \\
17 & -0.129089 & -0.052965 & 2.183699 \\
17 & -1.9691 & -1.581327 & -0.299718 \\
7 & 1.222521 & 1.472536 & -0.058882 \\
6 & 2.061891 & 2.261467 & 0.125873 \\
6 & 3.101613 & 3.248537 & 0.406917 \\
1 & 4.004623 & 3.037133 & -0.177093 \\
1 & 2.751441 & 4.256096 & 0.154802 \\
1 & 3.35833 & 3.225311 & 1.472324 \\
7 & 1.062679 & -1.425818 & -0.274789 \\
6 & 1.747755 & -2.364823 & -0.216358 \\
6 & 2.557919 & -3.573355 & -0.102398 \\
1 & 3.478202 & -3.48582 & -0.690725 \\
1 & 1.989059 & -4.437418 & -0.464421 \\
1 & 2.823144 & -3.745995 & 0.946899
\end{tabular}

\begin{tabular}{|c|c|c|c|}
\hline \multicolumn{4}{|c|}{$\begin{array}{l}14 \\
(\mathrm{~N} 2) \mathrm{Ru}(\mathrm{cCO}) \mathrm{Cl} 3(\mathrm{CH} 3 \mathrm{CN}) \quad \mathrm{E}(\mathrm{RB} 3 \mathrm{LYP})=-1831.45282109\end{array}$} \\
\hline 7 & & -0.487187 & 0.011507 \\
\hline 7 & 2.83891 & -0.931664 & -0.006693 \\
\hline 44 & -0.00595 & 0.243383 & 0.051843 \\
\hline 6 & 0.509302 & 1.874129 & -0.644704 \\
\hline 8 & 0.816988 & 2.924809 & -1.089237 \\
\hline 17 & 0.400575 & 0.969362 & 2.347839 \\
\hline 17 & -0.386558 & -0.707395 & -2.164511 \\
\hline 17 & -2.25767 & 1.063645 & 0.139025 \\
\hline 7 & -0.767294 & -1.531227 & 0.844255 \\
\hline 6 & -1.286489 & -2.475632 & 1.284819 \\
\hline 6 & -1.986757 & -3.632425 & 1.83514 \\
\hline 1 & -3.06712 & -3.510939 & 1.694388 \\
\hline 1 & -1.668877 & -4.551972 & 1.330165 \\
\hline 1 & -1.781923 & -3.728358 & 2.907681 \\
\hline
\end{tabular}


9

\begin{tabular}{lrrr} 
(N2)Ru(cCO)Cl4 & \multicolumn{2}{c}{$\mathrm{E}(\mathrm{RB} 3 \mathrm{LYP})=-2158.93203766$} & $\mathrm{x}$ \\
7 & -0.994321 & -0.010257 & 0.727321
\end{tabular}

$7 \quad-1.313364 \quad-1.101751 \quad 0.877867$

$\begin{array}{llll}44 & -0.456836 & 1.810209 & 0.474016\end{array}$

$\begin{array}{llll}6 & -2.008896 & 2.507205 & 1.134256\end{array}$

$\begin{array}{llll}8 & -3.027597 & 2.955293 & 1.567603\end{array}$

$\begin{array}{llll}17 & -1.474958 & 1.745425 & -1.778792\end{array}$

$\begin{array}{llll}17 & 1.674873 & 0.856247 & -0.432645\end{array}$

$\begin{array}{llll}17 & 0.480781 & 1.79819 & 2.762339\end{array}$

$\begin{array}{llll}17 & 0.299544 & 4.087021 & 0.122012\end{array}$

(N2)Ru ???

\begin{tabular}{|c|c|c|c|}
\hline \multicolumn{4}{|c|}{$\begin{array}{l}18 \\
(\mathrm{~N} 2) \mathrm{Ru}(\mathrm{cCO}) \mathrm{Cl}(\mathrm{NH} 3) 3 \quad \mathrm{E}(\mathrm{RB} 3 \mathrm{LYP})=-947.616307050\end{array}$} \\
\hline 7 & 0.303357 & -1.658107 & 0.32657 \\
\hline 7 & 0.974308 & -2.559203 & 0.529859 \\
\hline 44 & -0.845152 & -0.096239 & -0.013757 \\
\hline 6 & -1.844943 & -0.956872 & -1.343664 \\
\hline 8 & -2.485836 & -1.473443 & -2.179549 \\
\hline 17 & -2.12011 & 1.873712 & -0.283417 \\
\hline 7 & 0.482776 & 0.722665 & -1.502589 \\
\hline 1 & -0.004211 & 1.566455 & -1.833224 \\
\hline 1 & 1.406189 & 0.990804 & -1.148766 \\
\hline 7 & 0.218958 & 1.080493 & 1.520682 \\
\hline 1 & 1.21657 & 1.221189 & 1.332367 \\
\hline 1 & -0.263321 & 1.989098 & 1.477229 \\
\hline 1 & 0.140558 & 0.706625 & 2.47178 \\
\hline 1 & 0.624438 & 0.090649 & -2.296399 \\
\hline 7 & -2.290971 & -0.66979 & 1.480983 \\
\hline 1 & -1.930378 & -0.680228 & 2.440029 \\
\hline 1 & -3.024344 & 0.048236 & 1.410004 \\
\hline 1 & -2.707896 & -1.586994 & 1.294777 \\
\hline
\end{tabular}

12

(N2)Ru(cCO)F3(NH3) $\quad E(R B 3 L Y P)=-674.125910877 \quad x$

$\begin{array}{llll}7 & 1.525886 & -0.817567 & -0.42812\end{array}$

$\begin{array}{llll}7 & 2.414945 & -1.52454 & -0.609313\end{array}$

$\begin{array}{llll}44 & 0.008021 & 0.290619 & -0.10418\end{array}$

$\begin{array}{llll}6 & 0.800405 & 1.795377 & -0.772155\end{array}$

$\begin{array}{llll}8 & 1.328166 & 2.795167 & -1.166879\end{array}$

$\begin{array}{llll}9 & -0.746246 & -0.247176 & -1.869262\end{array}$ 


$\begin{array}{llll}9 & -1.621604 & 1.333979 & 0.395154 \\ 7 & 0.326544 & 0.664784 & 1.973459 \\ 1 & -0.405922 & 1.370168 & 2.109893 \\ 1 & -0.00537 & -0.266572 & 2.266128 \\ 9 & -0.825348 & -1.320261 & 0.845639 \\ 1 & 1.232007 & 0.931602 & 2.352493\end{array}$

14

$(\mathrm{N} 2) \mathrm{Ru}(\mathrm{cCO}) \mathrm{F} 3(\mathrm{CH} 3 \mathrm{CN}) \quad \mathrm{E}(\mathrm{RB} 3 \mathrm{LYP})=-750.279466781 \quad \mathrm{x}$

$\begin{array}{llll}7 & 1.803835 & -0.409682 & 0.087264 \\ 7 & 2.872827 & -0.837724 & 0.140484 \\ 44 & 0.014658 & 0.248037 & 0.019258 \\ 6 & 0.538459 & 1.85338 & -0.678365 \\ 8 & 0.862846 & 2.903858 & -1.132448 \\ 9 & -0.278229 & -0.616765 & -1.786624 \\ 9 & 0.121368 & 0.870511 & 1.943682 \\ 9 & -1.886375 & 0.788209 & 0.009615 \\ 7 & -0.745816 & -1.553147 & 0.830796 \\ 6 & -1.279267 & -2.485794 & 1.280284 \\ 6 & -1.987566 & -3.631906 & 1.844558 \\ 1 & -2.976241 & -3.319013 & 2.199876 \\ 1 & -2.119267 & -4.411696 & 1.085521 \\ 1 & -1.429231 & -4.052294 & 2.689077\end{array}$

\begin{tabular}{lllll}
9 & \multicolumn{4}{c}{$\mathrm{E}(\mathrm{RB} 3 \mathrm{LYP})=-717.355034016$} \\
(N2)Ru(cCO)F4 & $\mathrm{x}$ \\
7 & 1.503661 & -0.791459 & -0.348469 \\
7 & 2.415494 & -1.484667 & -0.526882 \\
44 & -0.013782 & 0.285566 & -0.042102 \\
6 & 0.781126 & 1.778168 & -0.708666 \\
8 & 1.314495 & 2.770613 & -1.150122 \\
9 & -0.788757 & -0.226632 & -1.857767 \\
9 & -1.684963 & 1.296078 & 0.331809 \\
9 & -0.91823 & -1.358011 & 0.709181 \\
9 & 0.601726 & 0.700727 & 1.857361
\end{tabular}

\begin{tabular}{lllll}
24 & \multicolumn{4}{l}{} \\
(N2)Ru(cCO)F(CH3CN)3 & $\mathrm{E}(\mathrm{RB} 3 \mathrm{LYP})=-815.767969204$ & $\mathrm{x}$ \\
7 & 1.019644 & 0.419849 & 2.062153 & \\
7 & 0.190465 & 0.49649 & 1.285988 \\
44 & -1.245743 & 0.597595 & -0.081944 \\
6 & -1.853628 & 2.275324 & 0.518798 \\
8 & -2.253205 & 3.316153 & 0.868757
\end{tabular}




$\begin{array}{llll}9 & -2.621921 & 0.581203 & -1.47483 \\ 7 & -2.586343 & -0.338817 & 1.106018 \\ 6 & -3.429379 & -0.857272 & 1.721323 \\ 6 & -4.503796 & -1.489942 & 2.480068 \\ 1 & -5.358799 & -1.688179 & 1.823483 \\ 1 & -4.164674 & -2.437546 & 2.91355 \\ 1 & -4.832998 & -0.831888 & 3.292465 \\ 7 & -0.03775 & 1.492067 & -1.433161 \\ 6 & 0.578003 & 2.018652 & -2.270812 \\ 6 & 1.327494 & 2.690419 & -3.327868 \\ 1 & 2.352568 & 2.306335 & -3.378952 \\ 1 & 0.839877 & 2.526194 & -4.295669 \\ 1 & 1.367336 & 3.768956 & -3.136624 \\ 7 & -0.681915 & -1.261334 & -0.857736 \\ 6 & -0.445627 & -2.286932 & -1.359581 \\ 6 & -0.16708 & -3.567676 & -2.002439 \\ 1 & -0.258367 & -4.386812 & -1.280255 \\ 1 & -0.878432 & -3.738017 & -2.818681 \\ 1 & 0.848475 & -3.576931 & -2.414045\end{array}$

\begin{tabular}{|c|c|c|c|}
\hline \multicolumn{3}{|c|}{ (N2)Ru(cCO)F(NH3)3 } & $E(R B 3 L Y P)=-587.236776347$ \\
\hline 7 & 0.307431 & -1.70722 & 0.312234 \\
\hline 7 & 0.971326 & -2.61404 & 0.518023 \\
\hline 44 & -0.813316 & -0.140648 & -0.005079 \\
\hline 6 & -1.80979 & -1.004464 & -1.335391 \\
\hline 8 & -2.444454 & -1.521916 & -2.176431 \\
\hline 9 & -1.826567 & 1.55239 & -0.160675 \\
\hline 7 & 0.445062 & 0.760765 & -1.490411 \\
\hline 1 & -0.076934 & 1.625715 & -1.689778 \\
\hline 1 & 1.393621 & 0.996665 & -1.184198 \\
\hline 7 & 0.207103 & 1.076853 & 1.508217 \\
\hline 1 & 1.197072 & 1.267225 & 1.32546 \\
\hline 1 & -0.334832 & 1.947567 & 1.411556 \\
\hline 1 & 0.136393 & 0.734003 & 2.471144 \\
\hline 1 & 0.523541 & 0.2127 & -2.351932 \\
\hline 7 & -2.301762 & -0.615864 & 1.465479 \\
\hline 1 & -1.968153 & -0.667617 & 2.43259 \\
\hline 1 & -2.94001 & 0.185918 & 1.365445 \\
\hline 1 & & -1.478984 & 1.266663 \\
\hline
\end{tabular}




\section{Trans-Carbonyl species}

\begin{tabular}{llll}
13 & & & \\
(N2)Ru(CO)5 & \multicolumn{2}{l}{$\mathrm{E}(\mathrm{RB} 3 \mathrm{LYP})=-770.372036704$} \\
7 & -0.287651 & -2.034109 & 0.382402 \\
7 & -0.425488 & -3.15328 & 0.431197 \\
44 & -0.029585 & 0.059781 & 0.290805 \\
6 & -0.086119 & 0.146179 & 2.317184 \\
8 & -0.118904 & 0.193061 & 3.464621 \\
6 & 0.210775 & 2.008263 & 0.20499 \\
6 & -2.043863 & 0.296733 & 0.225892 \\
6 & 1.982475 & -0.194566 & 0.357973 \\
8 & 3.12151 & -0.339294 & 0.397076 \\
8 & 0.351482 & 3.149214 & 0.155389 \\
8 & -3.184391 & 0.430192 & 0.190419 \\
6 & 0.023856 & -0.047549 & -1.735068 \\
8 & 0.053666 & -0.11167 & -2.88175
\end{tabular}

\begin{tabular}{|c|c|c|c|}
\hline \multicolumn{2}{|c|}{$(\mathrm{N} 2) \mathrm{Ru}(\mathrm{CO}) 2 \mathrm{Cl} 3$} & \multicolumn{2}{|c|}{$E(R B 3 L Y P)=-1$} \\
\hline 7 & 1.194675 & -1.766656 & 0.230648 \\
\hline 7 & 1.673732 & -2.765299 & 0.454988 \\
\hline 44 & 0.397649 & 0.092579 & -0.234282 \\
\hline 6 & -1.199574 & -0.212221 & 0.680556 \\
\hline 8 & -2.216545 & -0.390621 & 1.255954 \\
\hline 17 & -0.568634 & -0.928626 & -2.22355 \\
\hline 17 & 1.396582 & 0.927135 & 1.824727 \\
\hline 17 & 2.478686 & 0.488862 & -1.426658 \\
\hline 6 & -0.133191 & 1.795751 & -0.757647 \\
\hline 8 & -0.450127 & 2.874796 & -1.099644 \\
\hline
\end{tabular}

10

$(\mathrm{N} 2) \mathrm{Ru}(\mathrm{tCO}) 2 \mathrm{~F} 3 \quad \mathrm{E}(\mathrm{RB} 3 \mathrm{LYP})=-730.866558280$

$\begin{array}{llll}7 & 1.22307 & -1.771092 & 0.219467\end{array}$

$\begin{array}{llll}7 & 1.726001 & -2.758133 & 0.431747\end{array}$

$\begin{array}{llll}44 & 0.410204 & 0.132378 & -0.260391\end{array}$

$\begin{array}{llll}6 & -1.180748 & -0.158666 & 0.64532\end{array}$

$\begin{array}{llll}8 & -2.200963 & -0.350892 & 1.228417\end{array}$

$\begin{array}{llll}9 & -0.263443 & -0.766324 & -1.937005\end{array}$

$\begin{array}{llll}9 & 1.361473 & 0.764333 & 1.403861\end{array}$

$\begin{array}{llll}9 & 2.15412 & 0.327997 & -1.197716\end{array}$

$\begin{array}{llll}6 & -0.153723 & 1.806627 & -0.752112\end{array}$

$\begin{array}{llll}8 & -0.502739 & 2.889471 & -1.076497\end{array}$ 


\begin{tabular}{llll}
17 & \multicolumn{3}{c}{$\mathrm{E}(\mathrm{RB} 3 \mathrm{LYP})=-656.990955030$} \\
$(\mathrm{~N} 2) \mathrm{Ru}(\mathrm{tCO}) 3(\mathrm{NH} 3) 2$ & \multicolumn{3}{l}{-1.124833} \\
7 & 0.781364 & 1.027949 & -1.36 \\
7 & 1.883677 & 1.089246 & -1.364668 \\
44 & -1.255784 & 0.889185 & -0.612352 \\
6 & -0.951551 & 1.98519 & 0.983145 \\
8 & -0.772305 & 2.62946 & 1.925524 \\
7 & -1.603135 & 2.709852 & -1.790043 \\
1 & -2.376336 & 3.274143 & -1.412952 \\
1 & -0.772275 & 3.317146 & -1.789454 \\
1 & -1.82933 & 2.519047 & -2.774592 \\
6 & -3.133249 & 0.759029 & -0.184761 \\
8 & -4.259908 & 0.679795 & 0.064821 \\
6 & -0.897813 & -0.737525 & 0.419464 \\
8 & -0.682646 & -1.694859 & 1.029955 \\
7 & -1.575172 & -0.309095 & -2.42725 \\
1 & -0.742518 & -0.322815 & -3.031224 \\
1 & -1.788267 & -1.289908 & -2.20176 \\
1 & -2.360138 & 0.030057 & -2.998621
\end{tabular}

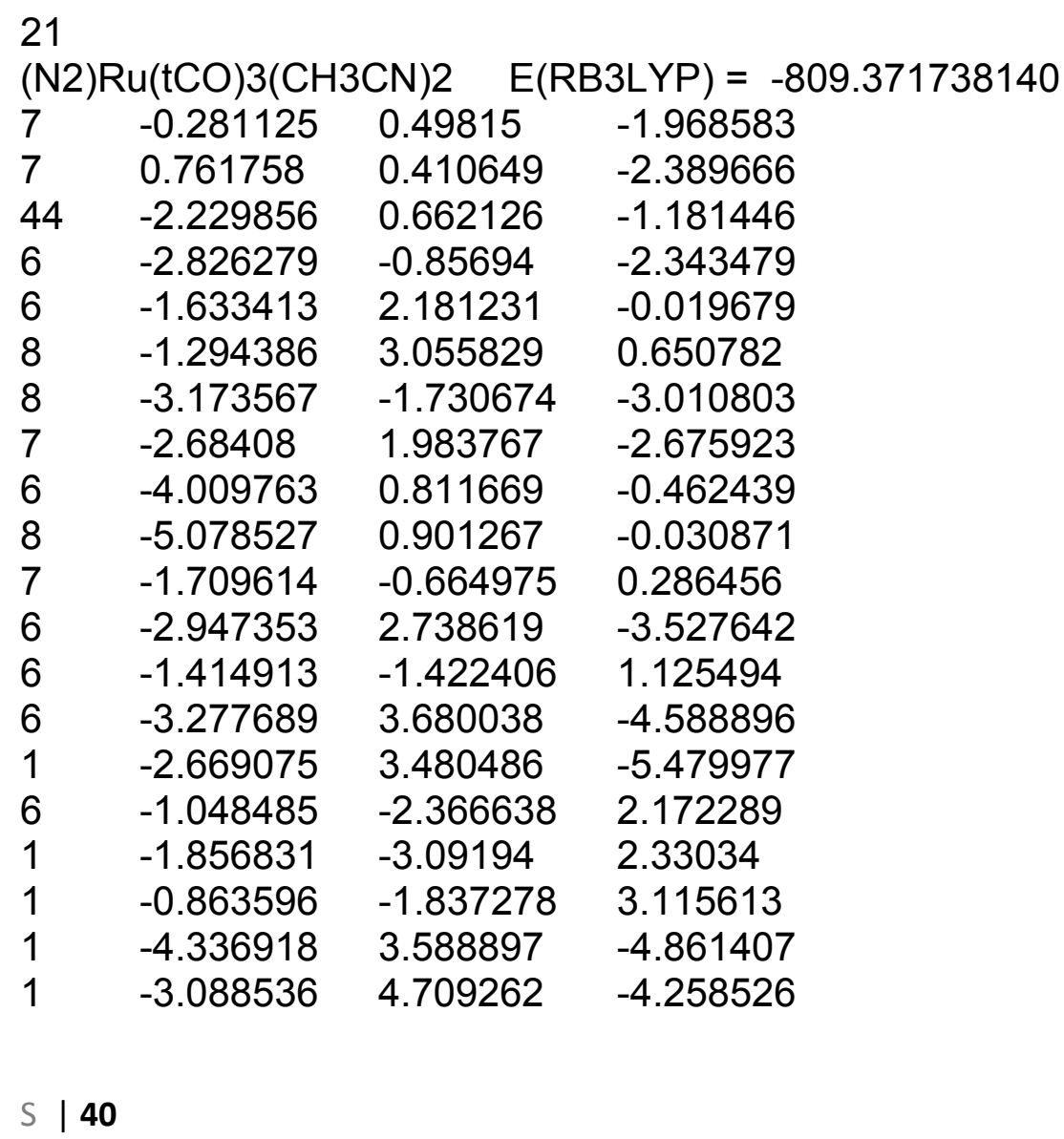


$\begin{array}{llll}1 & -0.138479 & -2.912636 & 1.892846\end{array}$

\begin{tabular}{|c|c|c|c|}
\hline \\
\hline 7 & 0.869384 & 0.979475 & -1.038737 \\
\hline 7 & 1.977249 & 1.038961 & -1.253263 \\
\hline 44 & -1.193708 & 0.871438 & -0.640634 \\
\hline 6 & -1.438015 & -0.272264 & -2.261108 \\
\hline 6 & -0.934251 & 2.016023 & 0.97697 \\
\hline 8 & -0.785014 & 2.67753 & 1.911485 \\
\hline 8 & -1.579982 & -0.932797 & -3.197445 \\
\hline 7 & -1.489628 & 2.659895 & -1.860721 \\
\hline 1 & -1.891478 & 3.436947 & -1.320756 \\
\hline 1 & -0.602737 & 2.997472 & -2.257409 \\
\hline 1 & -2.125488 & 2.48958 & -2.6504 \\
\hline 6 & -3.080556 & 0.773422 & -0.277838 \\
\hline 8 & -4.216198 & 0.714346 & -0.059857 \\
\hline 7 & -0.836308 & -0.913807 & 0.567494 \\
\hline 1 & -1.16586 & -0.79997 & 1.534645 \\
\hline 1 & -1.313492 & -1.741805 & 0.188515 \\
\hline 1 & 0.165642 & -1.141634 & 0.617085 \\
\hline
\end{tabular}

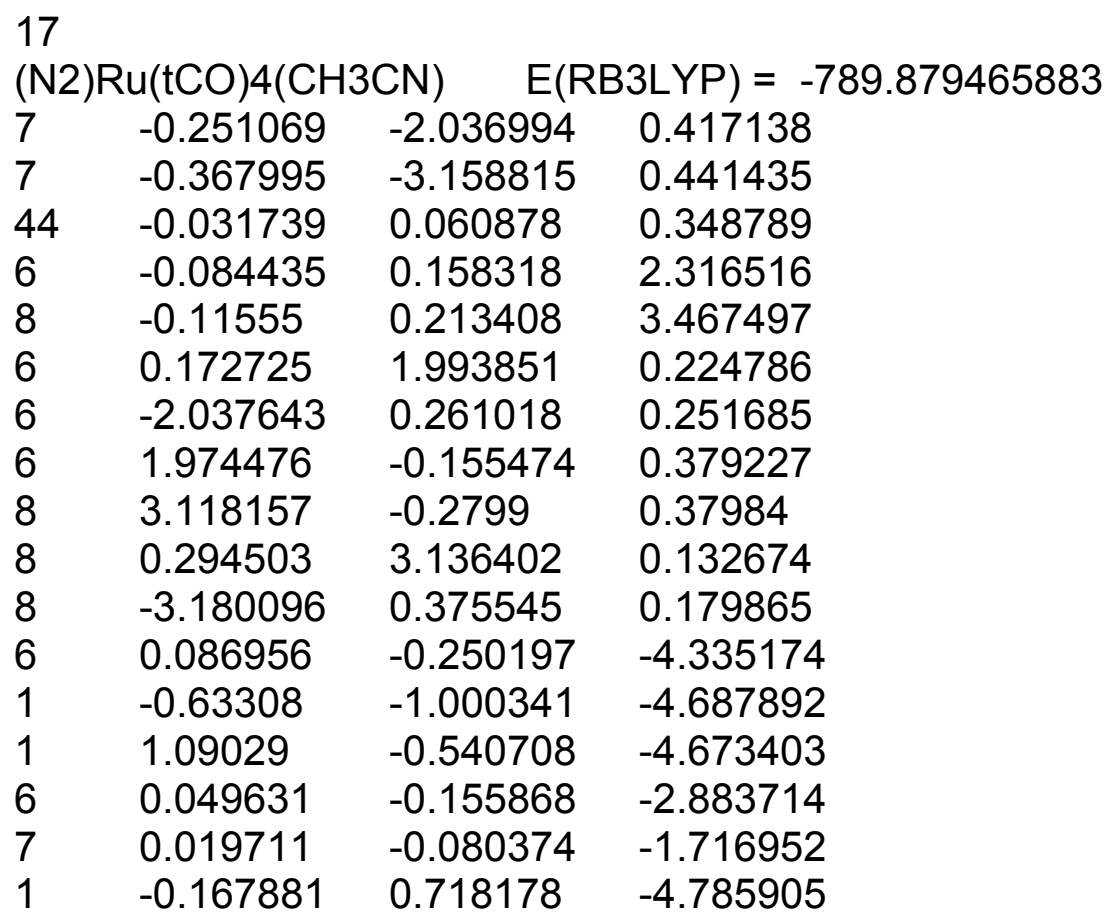




\begin{tabular}{llll}
15 & & & \\
(N2)Ru(tCO)4(NH3) & \multicolumn{2}{c}{$E(\mathrm{RB} 3 \mathrm{LYP})=-713.684039767$} \\
7 & 0.859408 & 0.990549 & -0.802556 \\
7 & 1.958083 & 0.954578 & -1.060596 \\
44 & -1.186954 & 1.009779 & -0.289895 \\
6 & -3.079645 & 1.050963 & 0.167404 \\
8 & -4.201007 & 1.077951 & 0.436223 \\
6 & -0.934673 & -0.621028 & 0.806762 \\
6 & -1.591601 & -0.131634 & -1.902381 \\
6 & -0.764084 & 2.158077 & 1.313297 \\
7 & -1.428553 & 2.816485 & -1.513985 \\
8 & -0.785723 & -1.569414 & 1.444167 \\
8 & -1.819919 & -0.789945 & -2.81869 \\
8 & -0.520352 & 2.806949 & 2.232352 \\
1 & -0.536193 & 3.104033 & -1.93873 \\
1 & -2.095387 & 2.681682 & -2.285752 \\
1 & -1.770161 & 3.618973 & -0.96827
\end{tabular}

\section{3}

$(\mathrm{N} 2) \mathrm{Ru}(\mathrm{tCO})(\mathrm{Acac}) 2 \quad \mathrm{E}(\mathrm{RB} 3 \mathrm{LYP})=-1008.20413169$

$\begin{array}{llll}7 & -0.096132 & 1.642628 & 0.932159 \\ 7 & -0.912917 & 0.859171 & 0.938395 \\ 44 & 1.407517 & 3.085005 & 0.920777 \\ 6 & 2.739776 & 4.362743 & 0.910495 \\ 8 & 3.592222 & 5.179808 & 0.903768 \\ 8 & 2.623621 & 1.716324 & 1.872248 \\ 6 & 2.906754 & 1.448181 & -1.125119 \\ 6 & 3.176023 & 1.149783 & -2.586994 \\ 1 & 3.918761 & 0.357641 & -2.716025 \\ 1 & 2.241697 & 0.855391 & -3.080617 \\ 1 & 3.529829 & 2.060701 & -3.086245 \\ 6 & 3.624353 & 0.758329 & -0.117592 \\ 6 & 3.455915 & 0.897394 & 1.281792 \\ 6 & 4.275542 & 0.046835 & 2.232182 \\ 1 & 4.929338 & -0.651199 & 1.701908 \\ 1 & 3.605222 & -0.515185 & 2.894082 \\ 1 & 4.360674 & 0.03667 & -0.450897 \\ 1 & 4.885713 & 0.698037 & 2.870777 \\ 8 & 0.082488 & 4.348813 & -0.030369 \\ 8 & 0.714858 & 3.706305 & 2.762413 \\ 6 & -0.696853 & 5.218756 & 0.559349 \\ 6 & -1.517562 & 6.067826 & -0.391434 \\ 1 & -2.200356 & 6.737975 & 0.138295 \\ 1 & -0.844879 & 6.663943 & -1.021209 \\ 1 & -2.093006 & 5.417832 & -1.06179\end{array}$




$\begin{array}{llll}6 & -0.819361 & 5.402397 & 1.958355 \\ 6 & -0.152752 & 4.664006 & 2.966484 \\ 6 & -0.430671 & 4.954818 & 4.428276 \\ 1 & -1.193603 & 5.727571 & 4.557009 \\ 1 & 0.496246 & 5.278042 & 4.918816 \\ 1 & -1.507778 & 6.170178 & 2.291017 \\ 1 & -0.756211 & 4.035714 & 4.930819 \\ 8 & 1.986513 & 2.35524 & -0.920312\end{array}$

\begin{tabular}{|c|c|c|c|}
\hline & $\mathrm{u}(\mathrm{tCO})\left(\mathrm{CH}_{3}\right.$ & & 0 \\
\hline 7 & -0.013387 & -2.051544 & 0.004537 \\
\hline & -0.033318 & -3.180029 & 0.004159 \\
\hline 44 & 0.023606 & 0.051583 & 0.005459 \\
\hline 6 & 0.05655 & 1.938979 & 0.00649 \\
\hline 8 & 0.076732 & 3.102156 & 0.007119 \\
\hline & -1.437126 & 0.048384 & -1.409188 \\
\hline 7 & -1.390644 & 0.046039 & 1.466571 \\
\hline 7 & 1.483918 & -0.004497 & 1.41945 \\
\hline 7 & 1.436838 & -0.002329 & -1.455656 \\
\hline 6 & -2.274716 & 0.054742 & -2.222103 \\
\hline 6 & -2.202037 & 0.05073 & 2.305644 \\
\hline 6 & 2.322598 & -0.028495 & 2.23091 \\
\hline 6 & 2.247874 & -0.025282 & -2.294776 \\
\hline 6 & -3.321101 & 0.067586 & -3.239049 \\
\hline 6 & -3.215935 & 0.061394 & 3.355018 \\
\hline 6 & 3.371617 & -0.053826 & 3.244914 \\
\hline 6 & 3.261434 & -0.04927 & -3.344253 \\
\hline 1 & -4.283008 & -0.226966 & -2.802859 \\
\hline 1 & -3.424632 & 1.073064 & -3.664608 \\
\hline 1 & -3.077363 & -0.630899 & -4.048428 \\
\hline 1 & -2.950597 & -0.644148 & 4.151357 \\
\hline 1 & -3.300941 & 1.064257 & 3.790701 \\
\hline 1 & -4.192524 & -0.22524 & 2.947118 \\
\hline 1 & 4.139075 & -0.792184 & 2.983833 \\
\hline 1 & 3.847031 & 0.931287 & 3.324176 \\
\hline 1 & 2.952612 & -0.31815 & 4.223087 \\
\hline 1 & 2.972614 & -0.746274 & -4.13994 \\
\hline 1 & 4.227997 & -0.367834 & -2.936153 \\
\hline 1 & 3.379687 & 0.949813 & -3.780871 \\
\hline
\end{tabular}

\section{3}

$(\mathrm{N} 2) \mathrm{Ru}(\mathrm{tCO})(\mathrm{CH} 3 \mathrm{CN})(\mathrm{NH} 3) 3 \quad \mathrm{E}(\mathrm{RB} 3 \mathrm{LYP})=-619.763476588$

$\begin{array}{llll}7 & 0.382094 & -0.975243 & 1.44412\end{array}$ 


$\begin{array}{llll}7 & 0.914793 & -1.594087 & 2.228749 \\ 44 & -0.652962 & 0.183651 & 0.057334 \\ 6 & -1.606478 & 1.238667 & -1.172032 \\ 8 & -2.207308 & 1.897686 & -1.926953 \\ 7 & 0.88233 & -0.236177 & -1.415831 \\ 1 & 1.438067 & 0.598026 & -1.640132 \\ 1 & 1.540713 & -0.957734 & -1.097531 \\ 7 & 0.445293 & 1.922449 & 0.75949 \\ 1 & 1.350582 & 1.681342 & 1.18048 \\ 1 & 0.622606 & 2.61568 & 0.023346 \\ 7 & -1.756254 & -1.58569 & -0.550164 \\ 1 & -1.920853 & -1.636862 & -1.562134 \\ 1 & -1.294149 & -2.458846 & -0.269781 \\ 1 & -2.678093 & -1.570238 & -0.097639 \\ 1 & -0.111771 & 2.39207 & 1.483398 \\ 1 & 0.483074 & -0.564023 & -2.303502 \\ 7 & -2.069593 & 0.541385 & 1.489418 \\ 6 & -2.889074 & 0.756489 & 2.297629 \\ 6 & -3.91226 & 1.028957 & 3.302768 \\ 1 & -4.716416 & 0.285186 & 3.243232 \\ 1 & -3.480175 & 0.993228 & 4.310367 \\ 1 & -4.347615 & 2.023261 & 3.143324\end{array}$

\begin{tabular}{llll}
19 & \multicolumn{4}{l}{ (N2)Ru(tCO)Cl2(CH3CN)2 $\mathrm{E}(\mathrm{RB} 3 \mathrm{LYP})=-1503.84743302$} \\
7 & 0.513323 & 0.44294 & 1.929138 \\
7 & -0.149145 & 0.459445 & 1.012999 \\
44 & -1.472837 & 0.539743 & -0.590425 \\
6 & -2.798498 & 0.685711 & -1.879723 \\
8 & -3.655704 & 0.78769 & -2.676925 \\
17 & -2.059859 & 2.717718 & 0.19459 \\
17 & -3.05315 & -0.52043 & 0.852625 \\
7 & -0.079107 & 1.466501 & -1.738902 \\
6 & 0.683675 & 2.087957 & -2.368156 \\
6 & 1.609302 & 2.916114 & -3.138006 \\
1 & 2.648088 & 2.671025 & -2.888421 \\
1 & 1.462285 & 2.76046 & -4.213054 \\
1 & 1.436778 & 3.974916 & -2.91228 \\
7 & -0.9405 & -1.330993 & -1.170799 \\
6 & -0.713205 & -2.442636 & -1.447535 \\
6 & -0.479948 & -3.850647 & -1.761182 \\
1 & -1.117415 & -4.482261 & -1.131482 \\
1 & -0.717027 & -4.054625 & -2.81177 \\
1 & 0.566852 & -4.120762 & -1.580448
\end{tabular}




\begin{tabular}{llll}
14 & \multicolumn{5}{l}{} \\
\multicolumn{5}{l}{$(\mathrm{N} 2) \mathrm{Ru}(\mathrm{tCO}) \mathrm{Cl} 3(\mathrm{CH} 3 \mathrm{CN})$} & $\mathrm{E}(\mathrm{RB} 3 \mathrm{LYP})=-1831.44577802$ \\
7 & 1.624808 & -0.534684 & 0.171137 \\
7 & 2.693958 & -0.907552 & 0.142018 \\
44 & -0.292426 & 0.221716 & 0.19111 \\
6 & -1.945488 & 1.030596 & 0.149165 \\
8 & -3.001536 & 1.562464 & 0.116835 \\
17 & 0.657924 & 2.231197 & -0.735142 \\
17 & 0.126846 & 0.967862 & 2.481303 \\
17 & -0.60544 & -0.761619 & -2.024379 \\
7 & -1.010036 & -1.473729 & 0.95897 \\
6 & -1.432947 & -2.466202 & 1.408896 \\
6 & -1.971603 & -3.701809 & 1.972654 \\
1 & -3.003518 & -3.857632 & 1.634626 \\
1 & -1.372139 & -4.563281 & 1.653585 \\
1 & -1.966707 & -3.660757 & 3.068862
\end{tabular}

12

$(\mathrm{N} 2) \mathrm{Ru}(\mathrm{tCO}) \mathrm{Cl} 3(\mathrm{NH} 3) \quad \mathrm{E}(\mathrm{RB} 3 \mathrm{LYP})=-1755.27702206$

$\begin{array}{llll}7 & 0.282396 & -1.019242 & 1.3905 \\ 7 & 0.848111 & -1.655843 & 2.141115 \\ 44 & -0.670836 & 0.225972 & 0.078588 \\ 6 & -1.427877 & 1.473634 & -1.03334 \\ 8 & -1.90658 & 2.29472 & -1.744989 \\ 17 & -2.45651 & 0.425797 & 1.746306 \\ 17 & 0.93653 & -0.511743 & -1.614142 \\ 17 & 0.725478 & 1.981477 & 0.995852 \\ 7 & -1.86131 & -1.419976 & -0.650335 \\ 1 & -2.428464 & -1.111685 & -1.439502 \\ 1 & -1.211156 & -2.147772 & -0.956526 \\ 1 & -2.460265 & -1.697394 & 0.132793\end{array}$

9

(N2)Ru(tCO)Cl4 $\quad E(R B 3 L Y P)=-2158.92607016$

$\begin{array}{llll}7 & -1.230352 & 0.289847 & 1.015594\end{array}$

$\begin{array}{llll}7 & -1.808147 & -0.618142 & 1.381315\end{array}$

$\begin{array}{llll}44 & -0.197813 & 1.913231 & 0.361101\end{array}$

$\begin{array}{llll}6 & 0.73104 & 3.374589 & -0.229325\end{array}$

$\begin{array}{llll}8 & 1.336549 & 4.326715 & -0.61443\end{array}$

$\begin{array}{llll}17 & -1.433893 & 1.735367 & -1.772169\end{array}$

$\begin{array}{llll}17 & 1.522482 & 0.367692 & -0.511127\end{array}$

$\begin{array}{llll}17 & -2.001562 & 3.328828 & 1.285411\end{array}$

$\begin{array}{llll}17 & 0.955136 & 1.960117 & 2.547321\end{array}$ 


\begin{tabular}{llll}
24 & \multicolumn{4}{l}{} \\
(N2)Ru(tCO)Cl(CH3CN)3 & $\mathrm{E}(\mathrm{RB} 3 \mathrm{LYP})=-1176.14689609$ \\
7 & 0.094975 & 0.287079 & 1.036918 \\
7 & 0.88702 & 0.302572 & 1.841941 \\
44 & -1.408802 & 0.352809 & -0.420311 \\
6 & -2.790566 & 0.540036 & -1.665051 \\
8 & -3.660689 & 0.678495 & -2.433485 \\
17 & -2.010271 & 2.455109 & 0.489547 \\
7 & -2.678687 & -0.498015 & 0.903394 \\
6 & -3.438883 & -0.89284 & 1.694284 \\
6 & -4.402285 & -1.34519 & 2.693333 \\
1 & -5.279772 & -1.785091 & 2.205723 \\
1 & -3.952631 & -2.096971 & 3.351791 \\
1 & -4.731055 & -0.495786 & 3.303367 \\
7 & -0.134016 & 1.333236 & -1.646222 \\
6 & 0.562493 & 1.986414 & -2.31519 \\
6 & 1.414756 & 2.840831 & -3.136456 \\
1 & 2.472699 & 2.633319 & -2.939633 \\
1 & 1.215712 & 2.671546 & -4.200816 \\
1 & 1.215873 & 3.894025 & -2.90632 \\
7 & -0.818102 & -1.47754 & -1.148214 \\
6 & -0.491225 & -2.516514 & -1.570606 \\
6 & -0.087863 & -3.815271 & -2.104879 \\
1 & -0.352497 & -4.616482 & -1.405366 \\
1 & -0.591749 & -4.006027 & -3.059538 \\
1 & 0.995196 & -3.841669 & -2.27106
\end{tabular}

\begin{tabular}{|c|c|c|c|}
\hline \multicolumn{4}{|c|}{$\begin{array}{l}18 \\
(\mathrm{~N} 2) \mathrm{Ru}(\mathrm{tCO}) \mathrm{Cl}(\mathrm{NH} 3) 3 \quad \mathrm{E}(\mathrm{RB} 3 \mathrm{LYP})=-947.601228258\end{array}$} \\
\hline (N2)Ru(tCO)Cl(NH3)3 & & -1.471596 & 0.543615 \\
\hline 7 & 0.998984 & -2.326693 & 0.912334 \\
\hline 44 & -0.914216 & 0.050961 & -0.035395 \\
\hline 6 & -2.127933 & 1.389041 & -0.474603 \\
\hline 8 & -2.907632 & 2.22893 & -0.737406 \\
\hline 17 & -2.395629 & -0.724609 & 1.65849 \\
\hline 7 & 0.528012 & 0.689632 & -1.57778 \\
\hline 1 & 0.82626 & 1.656463 & -1.415486 \\
\hline 1 & 1.365132 & 0.098264 & -1.58851 \\
\hline 7 & -0.051062 & 1.291372 & 1.514256 \\
\hline 1 & 0.915384 & 1.057232 & 1.761235 \\
\hline 1 & -0.104849 & 2.298781 & 1.338867 \\
\hline 7 & -1.930748 & -1.351203 & -1.333072 \\
\hline 1 & -2.392311 & -0.931559 & -2.145068 \\
\hline
\end{tabular}




$\begin{array}{llll}1 & -1.350471 & -2.130728 & -1.657692 \\ 1 & -2.654682 & -1.72205 & -0.700463 \\ 1 & -0.658768 & 1.066557 & 2.315411 \\ 1 & 0.101666 & 0.650557 & -2.508744\end{array}$

12

$\begin{array}{llll}(\mathrm{N} 2) \mathrm{Ru}(\mathrm{tCO}) \mathrm{F} 3(\mathrm{NH} 3) & \mathrm{E}(\mathrm{RB} 3 \mathrm{LYP})=-674.092001228 \\ 7 & 0.384075 & -1.020008 & 1.32243 \\ 7 & 0.997959 & -1.63458 & 2.052853 \\ 44 & -0.631586 & 0.241072 & 0.037686 \\ 6 & -1.431521 & 1.460982 & -1.042016 \\ 8 & -1.953512 & 2.270426 & -1.753979 \\ 9 & -2.184042 & 0.169991 & 1.427096 \\ 9 & 0.877071 & -0.168913 & -1.264413 \\ 9 & 0.498462 & 1.644254 & 0.945358 \\ 7 & -1.893812 & -1.381018 & -0.639984 \\ 1 & -2.299114 & -1.219925 & -1.5601 \\ 1 & -1.429177 & -2.288199 & -0.611101 \\ 1 & -2.565287 & -1.236137 & 0.13249\end{array}$

9

\begin{tabular}{llll} 
(N2)Ru(tCO)F4 & \multicolumn{2}{c}{$E(\mathrm{RB} 3 \mathrm{LYP})=-717.325072478$} \\
7 & -1.219192 & 0.307397 & 1.009712 \\
7 & -1.800748 & -0.601133 & 1.374779 \\
44 & -0.174486 & 1.948254 & 0.346715 \\
6 & 0.739947 & 3.388415 & -0.236744 \\
8 & 1.354519 & 4.355392 & -0.62782 \\
9 & -1.260523 & 1.726959 & -1.392406 \\
9 & 1.207093 & 0.588407 & -0.35447 \\
9 & -1.71785 & 3.054531 & 1.151684 \\
9 & 0.744682 & 1.910022 & 2.19224
\end{tabular}

\begin{tabular}{llll}
24 & \multicolumn{4}{l}{} \\
(N2)Ru(tCO)F(CH3CN)3 & $\mathrm{E}(\mathrm{RB} 3 \mathrm{LYP})=-815$. \\
7 & 0.108468 & 0.258733 & 1.031285 \\
7 & 0.891321 & 0.29738 & 1.843585 \\
44 & -1.398364 & 0.314415 & -0.437279 \\
6 & -2.769327 & 0.479821 & -1.689146 \\
8 & -3.634775 & 0.601691 & -2.467531 \\
9 & -1.8375 & 2.057404 & 0.376573 \\
7 & -2.676892 & -0.500481 & 0.896247 \\
6 & -3.444878 & -0.853079 & 1.69967 \\
6 & -4.416351 & -1.258819 & 2.710793
\end{tabular}




$\begin{array}{llll}1 & -5.305534 & -1.688063 & 2.235015 \\ 1 & -3.983372 & -2.006825 & 3.384607 \\ 1 & -4.722693 & -0.388587 & 3.30279 \\ 7 & -0.136967 & 1.328355 & -1.64459 \\ 6 & 0.542809 & 2.019529 & -2.292494 \\ 6 & 1.374768 & 2.914372 & -3.091162 \\ 1 & 2.437324 & 2.724597 & -2.901395 \\ 1 & 1.17766 & 2.768857 & -4.159416 \\ 1 & 1.153643 & 3.956553 & -2.833141 \\ 7 & -0.820697 & -1.504366 & -1.170338 \\ 6 & -0.491495 & -2.543598 & -1.590959 \\ 6 & -0.083881 & -3.842584 & -2.121614 \\ 1 & -0.345721 & -4.643192 & -1.4203 \\ 1 & -0.587121 & -4.037803 & -3.075745 \\ 1 & 0.999205 & -3.866236 & -2.288304\end{array}$

\begin{tabular}{llll}
18 & \multicolumn{4}{l}{} \\
\multicolumn{1}{l}{$(\mathrm{N} 2) \mathrm{Ru}(\mathrm{tCO}) \mathrm{F}(\mathrm{NH} 3) 3$} & $\mathrm{E}(\mathrm{RB} 3 \mathrm{LYP})=-587.217953455$ \\
7 & 0.379831 & -1.460222 & 0.513808 \\
7 & 1.02479 & -2.316255 & 0.883119 \\
44 & -0.887596 & 0.069261 & -0.068886 \\
6 & -2.066091 & 1.419942 & -0.543336 \\
8 & -2.815272 & 2.27719 & -0.844101 \\
9 & -2.084935 & -0.625891 & 1.366644 \\
7 & 0.544292 & 0.707321 & -1.608195 \\
1 & 0.79342 & 1.693796 & -1.486739 \\
1 & 1.409376 & 0.158702 & -1.574173 \\
7 & -0.116982 & 1.24937 & 1.560248 \\
1 & 0.847681 & 1.054199 & 1.843108 \\
1 & -0.228217 & 2.262698 & 1.470413 \\
7 & -1.981767 & -1.36053 & -1.251646 \\
1 & -2.534256 & -0.97671 & -2.022802 \\
1 & -1.446106 & -2.159155 & -1.603757 \\
1 & -2.621074 & -1.681881 & -0.507993 \\
1 & -0.76083 & 0.904745 & 2.289326 \\
1 & 0.14255 & 0.602771 & -2.545052
\end{tabular}

\section{Poly-cis-dinitrogen species}

12

(N2)Ru-Cl-n24 $\quad E(R B 3 L Y P)=-1102.46426314$

$\begin{array}{llll}7 & 0.094261 & -1.432031 & 0.979062\end{array}$ 


$\begin{array}{llll}7 & -0.299243 & -2.165701 & 1.746896 \\ 44 & 0.813063 & -0.09055 & -0.42597 \\ 7 & 0.484827 & 1.477775 & 0.787443 \\ 7 & 0.3197 & 2.392546 & 1.430785 \\ 7 & -1.009295 & 0.158395 & -1.236337 \\ 7 & -2.012279 & 0.333417 & -1.72759 \\ 7 & 2.691035 & -0.236838 & 0.27533 \\ 7 & 3.762831 & -0.282752 & 0.631822 \\ 7 & 1.196886 & -1.555146 & -1.747443 \\ 7 & 1.431851 & -2.340707 & -2.525948 \\ 17 & 1.619026 & 1.41766 & -2.005039\end{array}$

11

(N2)Ru-Cl2cisn23 $\quad E(R B 3 L Y P)=-1453.49568076$

$\begin{array}{llll}7 & 0.122977 & -1.390397 & 0.919406\end{array}$

$\begin{array}{llll}7 & -0.254897 & -2.190829 & 1.631786\end{array}$

$\begin{array}{llll}44 & 0.80519 & -0.02194 & -0.389464\end{array}$

$\begin{array}{llll}7 & 0.470523 & 1.536605 & 0.838841\end{array}$

$\begin{array}{llll}7 & 0.296223 & 2.440758 & 1.504557\end{array}$

$\begin{array}{llll}7 & -0.97307 & 0.164519 & -1.243136\end{array}$

$\begin{array}{llll}7 & -1.957249 & 0.26527 & -1.794014\end{array}$

$\begin{array}{llll}7 & 2.671048 & -0.224514 & 0.246414\end{array}$

$\begin{array}{llll}7 & 3.757439 & -0.344339 & 0.542406\end{array}$

$\begin{array}{llll}17 & 1.216823 & -1.807447 & -1.861652\end{array}$

$\begin{array}{llll}17 & 1.610155 & 1.535908 & -1.954647\end{array}$

10

\begin{tabular}{llll}
\multicolumn{2}{c}{ (N2)Ru-Cl3n22 } & \multicolumn{2}{c}{ E(RB3LYP $)=-1804.37038753$} \\
7 & -0.359272 & -0.751544 & 1.218668
\end{tabular}

$\begin{array}{llll}7 & -1.059091 & -1.198615 & 2.001112\end{array}$

$\begin{array}{llll}44 & 0.887441 & -0.007978 & -0.098716\end{array}$

$\begin{array}{llll}7 & 0.105527 & 1.788445 & -0.033961\end{array}$

$\begin{array}{llll}7 & -0.318904 & 2.846773 & 0.007243\end{array}$

$\begin{array}{llll}7 & -0.184311 & -0.571525 & -1.640279\end{array}$

$\begin{array}{llll}7 & -0.780434 & -0.911983 & -2.551653\end{array}$

$\begin{array}{llll}17 & 1.902636 & -2.163657 & -0.170688\end{array}$

$\begin{array}{llll}17 & 2.248305 & 0.652777 & 1.743543\end{array}$

$\begin{array}{llll}17 & 2.457504 & 0.864778 & -1.665959\end{array}$

19

(N2)Ru-cn22-(NH3)3 $\quad E(R B 3 L Y P)=-592.636382771$

$\begin{array}{llll}7 & -0.937344 & -1.38655 & 0.969943\end{array}$

$\begin{array}{llll}7 & -1.569178 & -2.154839 & 1.515655\end{array}$ 


$\begin{array}{llll}44 & 0.134568 & 0.013268 & 0.004063 \\ 7 & -0.915637 & 1.465188 & 0.915541 \\ 7 & -1.536698 & 2.272065 & 1.416052 \\ 7 & -1.18394 & -0.006868 & -1.513686 \\ 7 & -1.951779 & -0.02672 & -2.348723 \\ 7 & 1.27854 & 1.527744 & -1.012344 \\ 1 & 2.1536 & 1.184121 & -1.428185 \\ 1 & 1.528727 & 2.303127 & -0.384352 \\ 1 & 0.732035 & 1.944224 & -1.77839 \\ 7 & 1.252371 & -1.537484 & -0.987336 \\ 1 & 1.330689 & -1.367631 & -1.998905 \\ 1 & 0.781666 & -2.446924 & -0.885736 \\ 1 & 2.207868 & -1.65933 & -0.62801 \\ 7 & 1.54582 & 0.014199 & 1.630395 \\ 1 & 2.468657 & 0.393521 & 1.383053 \\ 1 & 1.699625 & -0.93284 & 2.00157 \\ 1 & 1.19696 & 0.576947 & 2.418114\end{array}$

\begin{tabular}{|c|c|c|c|}
\hline & $\mathrm{u}-\mathrm{F} 2 \operatorname{cisn} 23$ & E(RB3LYF & $=-732.729$ \\
\hline 7 & 0.13922 & -1.390733 & 0.879468 \\
\hline 7 & -0.234042 & -2.21287 & 1.572423 \\
\hline 44 & 0.808702 & -0.022917 & -0.398595 \\
\hline 7 & 0.48589 & 1.530339 & 0.799887 \\
\hline 7 & 0.323688 & 2.454248 & 1.444431 \\
\hline 7 & -0.957422 & 0.161951 & -1.279217 \\
\hline 7 & -1.916116 & 0.260643 & -1.873086 \\
\hline 7 & 2.684764 & -0.228582 & 0.206996 \\
\hline 7 & 3.783001 & -0.353674 & 0.451303 \\
\hline 9 & 1.166816 & -1.477194 & -1.644779 \\
\hline 9 & 1.48066 & 1.242384 & -1.718335 \\
\hline
\end{tabular}

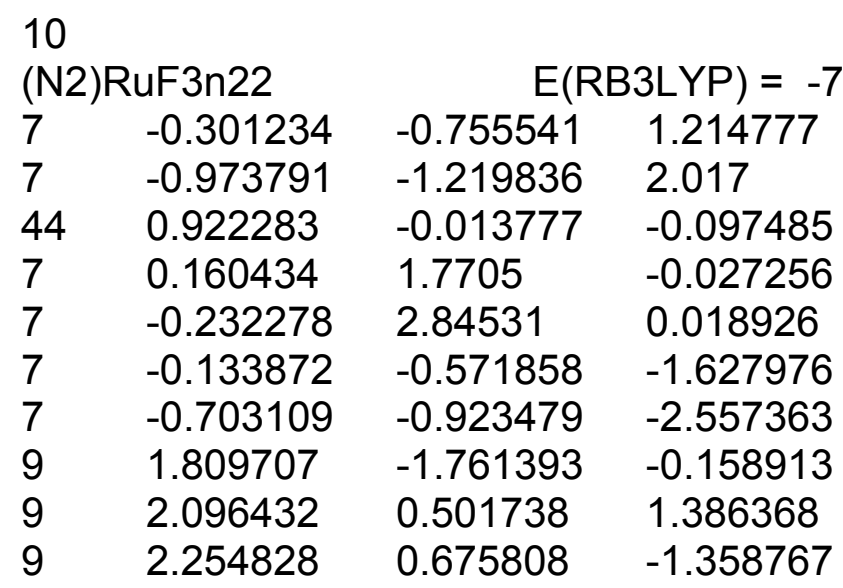




\begin{tabular}{llll}
25 & \multicolumn{3}{c}{ E(RB3LYP $)=-821.212876922$} \\
(N2)Run22(CH3CN)3 & \multicolumn{2}{c}{ R 2.154254} & -0.064328 \\
7 & 0.484595 & -2.1548 & 0.027943 \\
7 & 0.676085 & -3.265885 & -0.22864 \\
44 & 0.143674 & -0.175686 & -0.761238 \\
7 & -0.036672 & -0.023828 & 1.764 \\
7 & 0.31834 & -0.294276 & -2.221236 \\
7 & 0.415044 & -0.34989 & -3.346906 \\
7 & -0.198017 & 1.809087 & -0.39386 \\
6 & -0.394683 & 2.955602 & -0.489725 \\
6 & -0.639789 & 4.388035 & -0.6101 \\
1 & -1.646984 & 4.634742 & -0.252819 \\
1 & 0.089079 & 4.951492 & -0.014871 \\
1 & -0.553626 & 4.702506 & -1.657473 \\
7 & 2.142862 & 0.1737 & -0.07449 \\
6 & 3.292498 & 0.357595 & 0.015671 \\
6 & 4.72987 & 0.57522 & 0.129371 \\
1 & 4.942687 & 1.337136 & 0.88907 \\
1 & 5.233247 & -0.355607 & 0.418473 \\
1 & 5.142365 & 0.911235 & -0.829803 \\
7 & -1.85302 & -0.539451 & -0.381717 \\
6 & -2.99575 & -0.76349 & -0.46881 \\
6 & -4.420524 & -1.054405 & -0.577099 \\
1 & -4.987895 & -0.465674 & 0.153959 \\
1 & -4.78472 & -0.810581 & -1.582591 \\
1 & -4.60652 & -2.118737 & -0.387467 \\
7 & -0.140594 & 0.073587 & 2.883416
\end{tabular}

\begin{tabular}{llll}
21 & \multicolumn{3}{l}{} \\
(N2)Run23(CH3CN)2 & \multicolumn{2}{l}{$\mathrm{E}(\mathrm{RB} 3 \mathrm{LYP})=-7$} \\
7 & 0.478285 & -2.169649 & -0.045725 \\
7 & 0.660643 & -3.281947 & 0.045795 \\
44 & 0.13683 & -0.173625 & -0.211173 \\
7 & -0.0806 & -0.029688 & 1.787906 \\
7 & 0.319419 & -0.293576 & -2.21526 \\
7 & 0.409133 & -0.352239 & -3.340582 \\
7 & -0.20671 & 1.81092 & -0.376228 \\
6 & -0.391292 & 2.960201 & -0.470695 \\
6 & -0.6101 & 4.395855 & -0.587982 \\
1 & -1.5566 & 4.679279 & -0.110996 \\
1 & 0.205248 & 4.945459 & -0.100635 \\
1 & -0.647143 & 4.69069 & -1.644324 \\
7 & 2.12518 & 0.207765 & -0.038237
\end{tabular}




$\begin{array}{llll}7 & -1.841552 & -0.548332 & -0.383891 \\ 6 & -2.982449 & -0.777122 & -0.483196 \\ 6 & -4.403757 & -1.07277 & -0.606618 \\ 1 & -4.982485 & -0.472647 & 0.106689 \\ 1 & -4.753912 & -0.846305 & -1.621585 \\ 1 & -4.590214 & -2.13464 & -0.401735 \\ 7 & -0.215471 & 0.059991 & 2.906657 \\ 7 & 3.23035 & 0.427523 & 0.057352\end{array}$

\begin{tabular}{|c|c|c|c|}
\hline \\
\hline 7 & -0.064208 & -1.613119 & -0.897176 \\
\hline 7 & -0.151436 & -2.612836 & -1.46702 \\
\hline 44 & 0.061945 & 0.053632 & -0.010573 \\
\hline 9 & 0.047318 & 1.855123 & 0.865356 \\
\hline 9 & 2.029273 & 0.089139 & -0.330267 \\
\hline 9 & -0.360128 & 0.956747 & -1.747855 \\
\hline 7 & 0.339431 & -0.722452 & 1.692414 \\
\hline 7 & 0.505386 & -1.164173 & 2.745573 \\
\hline 7 & -2.024881 & 0.478317 & 0.168234 \\
\hline 1 & -1.919856 & 1.30481 & 0.770126 \\
\hline 1 & -2.16716 & 0.758432 & -0.810293 \\
\hline 1 & -2.70101 & -0.201213 & 0.509433 \\
\hline
\end{tabular}

\begin{tabular}{|c|c|c|c|}
\hline & un2 $(\mathrm{CH} 3 \mathrm{Cl}$ & $4 \quad E(F$ & $3 L Y P)=-844.509194056$ \\
\hline 7 & 0.480145 & -2.136258 & -0.048309 \\
\hline 7 & 0.671076 & -3.248566 & 0.05283 \\
\hline 44 & 0.142142 & -0.175314 & -0.209624 \\
\hline 7 & -0.034076 & -0.06018 & 1.800419 \\
\hline 7 & 0.313921 & -0.274978 & -2.196285 \\
\hline 7 & 0.409847 & -0.321845 & -3.324326 \\
\hline 6 & -0.133807 & -0.005185 & 2.961715 \\
\hline 6 & -0.257053 & 0.052695 & 4.414681 \\
\hline 1 & -0.025097 & -0.924645 & 4.854875 \\
\hline 1 & 0.436021 & 0.794558 & 4.828849 \\
\hline 1 & -1.278224 & 0.330234 & 4.701768 \\
\hline 7 & -0.201069 & 1.810717 & -0.359178 \\
\hline 6 & -0.397421 & 2.95666 & -0.45835 \\
\hline 6 & -0.641345 & 4.389069 & -0.593903 \\
\hline 1 & -1.637721 & 4.645753 & -0.215029 \\
\hline 1 & 0.104975 & 4.96 & -0.028859 \\
\hline 1 & -0.582336 & 4.68632 & -1.647926 \\
\hline 7 & 2.137678 & 0.175819 & -0.059301 \\
\hline
\end{tabular}




$\begin{array}{llll}6 & 3.28817 & 0.359834 & 0.013301 \\ 6 & 4.729115 & 0.576709 & 0.093102 \\ 1 & 4.955121 & 1.421772 & 0.754017 \\ 1 & 5.227343 & -0.317614 & 0.4863 \\ 1 & 5.137168 & 0.792163 & -0.901749 \\ 7 & -1.851072 & -0.536142 & -0.367643 \\ 6 & -2.991943 & -0.759888 & -0.473044 \\ 6 & -4.414602 & -1.051905 & -0.616248 \\ 1 & -5.010885 & -0.376489 & 0.00849 \\ 1 & -4.725307 & -0.927539 & -1.660598 \\ 1 & -4.623566 & -2.08474 & -0.312594\end{array}$

\section{Mixed RuCO- $\mathrm{N}_{2}$-CisCO species [Figs. 16 and S14]}

\begin{tabular}{llll}
21 & & & \\
\multicolumn{3}{l}{ ruco-n2(NH3)4co } & \multicolumn{2}{l}{$E(\mathrm{RB} 3 \mathrm{LYP})=-543.567168895$} \\
6 & 1.021101 & 0.631698 & -0.464384 \\
8 & 1.787045 & -0.094097 & -0.966355 \\
44 & -0.213365 & 1.804636 & 0.353046 \\
7 & -1.031765 & 0.306152 & 1.370296 \\
7 & -1.499944 & -0.554517 & 1.947029 \\
7 & -1.662988 & 3.165003 & 1.354582 \\
1 & -2.617517 & 3.051586 & 0.990667 \\
1 & -1.719054 & 2.950427 & 2.358753 \\
1 & -1.432032 & 4.16319 & 1.276082 \\
7 & 1.180954 & 2.160085 & 1.989299 \\
1 & 0.939127 & 2.967605 & 2.576375 \\
1 & 2.1408 & 2.304973 & 1.652074 \\
1 & 1.216072 & 1.3395 & 2.607783 \\
7 & -1.626469 & 1.475711 & -1.27178 \\
1 & -2.026792 & 0.530936 & -1.205853 \\
1 & -2.415317 & 2.13347 & -1.286072 \\
1 & -1.171934 & 1.532142 & -2.191364 \\
1 & 1.265782 & 4.039856 & -0.157873 \\
1 & 1.124158 & 3.197571 & -1.562526 \\
1 & -0.122855 & 4.144073 & -1.043938 \\
7 & 0.605873 & 3.492987 & -0.725527
\end{tabular}

33

ruco-n2(Acac)2(tCO) $\quad E(R B 3 L Y P)=-1008.22131311$

$\begin{array}{llll}6 & -0.243478 & 0.638666 & -0.504702\end{array}$

$\begin{array}{llll}8 & -0.582491 & -0.135306 & -1.328805\end{array}$

$\begin{array}{llll}44 & 0.296716 & 1.891333 & 0.762106\end{array}$

$\begin{array}{llll}7 & -1.057674 & 1.330165 & 2.043277\end{array}$

$\begin{array}{llll}7 & -1.822685 & 1.005832 & 2.824291\end{array}$ 


$\begin{array}{llll}8 & 1.727725 & 2.621912 & -0.479527 \\ 8 & 1.640404 & 0.600166 & 1.622638 \\ 6 & 2.987436 & 2.25748 & -0.500407 \\ 6 & 3.829411 & 2.995673 & -1.520405 \\ 1 & 4.87492 & 2.676427 & -1.498115 \\ 1 & 3.421577 & 2.826233 & -2.524811 \\ 1 & 3.775938 & 4.074493 & -1.328297 \\ 6 & 3.572021 & 1.274679 & 0.327299 \\ 6 & 2.910822 & 0.522841 & 1.329771 \\ 6 & 3.686346 & -0.472436 & 2.168761 \\ 1 & 4.739056 & -0.523758 & 1.877669 \\ 1 & 3.234683 & -1.467447 & 2.071969 \\ 1 & 4.630772 & 1.088178 & 0.193952 \\ 1 & 3.617824 & -0.187573 & 3.226118 \\ 8 & -0.964702 & 3.31112 & -0.03225 \\ 8 & 0.984439 & 3.292364 & 2.12573 \\ 6 & -1.011076 & 4.576847 & 0.292102 \\ 6 & -1.970132 & 5.397336 & -0.547716 \\ 1 & -2.014108 & 6.440172 & -0.221559 \\ 1 & -1.6542 & 5.361158 & -1.59795 \\ 1 & -2.974111 & 4.958455 & -0.497272 \\ 6 & -0.267891 & 5.197113 & 1.32533 \\ 6 & 0.663166 & 4.559875 & 2.176544 \\ 6 & 1.376482 & 5.353003 & 3.253056 \\ 1 & 1.054068 & 6.397677 & 3.27815 \\ 1 & 2.45937 & 5.31363 & 3.079092 \\ 1 & -0.429502 & 6.257563 & 1.476913 \\ 1 & 1.190616 & 4.892809 & 4.231456\end{array}$

\begin{tabular}{llll}
12 & & & \\
ruco-n2(tCO)aF3 & \multicolumn{2}{l}{$\mathrm{E}(\mathrm{RB} 3 \mathrm{LYP})=$} & -674.127418732 \\
6 & -0.075079 & -0.049703 & 1.821306 \\
8 & -0.119665 & -0.081195 & 3.012195 \\
44 & -0.001416 & 0.042377 & -0.011607 \\
7 & 0.137741 & -1.83727 & -0.205384 \\
7 & 0.236988 & -2.974524 & -0.371238 \\
9 & -0.127529 & 2.036842 & -0.066857 \\
9 & -1.998093 & -0.021031 & -0.361588 \\
9 & 2.006472 & 0.241777 & -0.152438 \\
7 & 0.035207 & 0.466644 & -2.130819 \\
1 & 0.017895 & 1.481626 & -1.962444 \\
1 & 0.885937 & 0.153106 & -2.593688 \\
1 & -0.832039 & 0.125074 & -2.543292
\end{tabular}




\begin{tabular}{llll}
23 & \multicolumn{4}{l}{$l$} \\
ruco-n2(tCO)(CH3CN)-(NH3)3 & E(RBLYP $)=-619.764243226$ \\
6 & -0.0742 & -1.822362 & -0.392631 \\
8 & -0.021936 & -2.987585 & -0.363188 \\
44 & -0.158998 & 0.071345 & -0.412808 \\
7 & 1.829644 & 0.20016 & -0.545759 \\
7 & 2.962362 & 0.259155 & -0.592508 \\
7 & -0.298023 & 0.089784 & -2.57286 \\
1 & -0.183517 & 1.029181 & -2.972417 \\
1 & 0.441163 & -0.496581 & -2.980919 \\
7 & -0.257354 & 2.282133 & -0.380545 \\
1 & 0.647367 & 2.698656 & -0.634113 \\
1 & -0.962329 & 2.699005 & -1.00043 \\
7 & -2.299624 & -0.012927 & -0.225995 \\
1 & -2.765567 & 0.883193 & -0.411754 \\
1 & -2.731255 & -0.713571 & -0.840967 \\
1 & -2.535121 & -0.282109 & 0.737517 \\
1 & -0.466646 & 2.593568 & 0.575808 \\
1 & -1.189777 & -0.281145 & -2.922371 \\
7 & -0.02688 & 0.121475 & 1.631325 \\
6 & 0.115817 & 0.088775 & 2.792908 \\
6 & 0.31218 & 0.030001 & 4.237896 \\
1 & -0.567034 & 0.417835 & 4.766586 \\
1 & 0.479345 & -1.007681 & 4.553173 \\
1 & 1.18611 & 0.625992 & 4.528866
\end{tabular}

\begin{tabular}{llll}
29 & \multicolumn{3}{l}{} \\
ruco-n2(tCO)(CH3CN)4 & $\mathrm{E}(\mathrm{RB} 3 \mathrm{LYP})=-8$ \\
6 & 0.309438 & -0.270707 & -2.152751 \\
8 & 0.406685 & -0.314201 & -3.310675 \\
44 & 0.145827 & -0.176894 & -0.256616 \\
7 & 0.482722 & -2.143976 & -0.070229 \\
7 & 0.67185 & -3.254021 & 0.04747 \\
7 & -0.036556 & -0.059003 & 1.819996 \\
6 & -0.135111 & -0.007963 & 2.982437 \\
6 & -0.256852 & 0.046349 & 4.435628 \\
1 & -0.023967 & -0.931895 & 4.873144 \\
1 & 0.436286 & 0.787661 & 4.850503 \\
1 & -1.277872 & 0.322786 & 4.724103 \\
7 & -0.198774 & 1.811995 & -0.387144 \\
6 & -0.395712 & 2.958198 & -0.477546 \\
6 & -0.640413 & 4.391121 & -0.603647 \\
1 & -1.636488 & 4.645028 & -0.222089 \\
1 & 0.106349 & 4.959012 & -0.036099 \\
1 & -0.582689 & 4.69407 & -1.656174
\end{tabular}




$\begin{array}{llll}7 & 2.142169 & 0.1757 & -0.07867 \\ 6 & 3.291067 & 0.361645 & 0.007436 \\ 6 & 4.730592 & 0.580421 & 0.102106 \\ 1 & 4.949131 & 1.425441 & 0.76558 \\ 1 & 5.226134 & -0.313584 & 0.499499 \\ 1 & 5.147452 & 0.796743 & -0.888977 \\ 7 & -1.852818 & -0.536841 & -0.388431 \\ 6 & -2.99476 & -0.759751 & -0.47912 \\ 6 & -4.418931 & -1.050381 & -0.607084 \\ 1 & -5.008487 & -0.372731 & 0.021583 \\ 1 & -4.738822 & -0.928324 & -1.648997 \\ 1 & -4.626038 & -2.082295 & -0.298961\end{array}$

19

ruco-n2(tCO)Cl2(CH3CN)2 E(RB3LYP) $=-1503.85397232$

$\begin{array}{llll}6 & -0.346969 & 0.158714 & -2.102011 \\ 8 & -0.398699 & 0.193088 & -3.279676 \\ 44 & -0.261457 & 0.080426 & -0.231991 \\ 7 & -1.659506 & 1.440074 & 0.00723 \\ 7 & -2.489142 & 2.193484 & 0.187521 \\ 17 & -0.129089 & -0.052965 & 2.183699 \\ 17 & -1.9691 & -1.581327 & -0.299718 \\ 7 & 1.222521 & 1.472536 & -0.058882 \\ 6 & 2.061891 & 2.261467 & 0.125873 \\ 6 & 3.101613 & 3.248537 & 0.406917 \\ 1 & 4.004623 & 3.037133 & -0.177093 \\ 1 & 2.751441 & 4.256096 & 0.154802 \\ 1 & 3.35833 & 3.225311 & 1.472324 \\ 7 & 1.062679 & -1.425818 & -0.274789 \\ 6 & 1.747755 & -2.364823 & -0.216358 \\ 6 & 2.557919 & -3.573355 & -0.102398 \\ 1 & 3.478202 & -3.48582 & -0.690725 \\ 1 & 1.989059 & -4.437418 & -0.464421 \\ 1 & 2.823144 & -3.745995 & 0.946899\end{array}$

14

ruco-n2 $(\mathrm{tCO}) \mathrm{Cl} 3(\mathrm{CH} 3 \mathrm{CN}) \quad \mathrm{E}(\mathrm{RB} 3 \mathrm{LYP})=-1831.45282109$

$\begin{array}{llll}6 & 0.509302 & 1.874129 & -0.644704 \\ 8 & 0.816988 & 2.924809 & -1.089237 \\ 44 & -0.00595 & 0.243383 & 0.051843 \\ 7 & 1.78425 & -0.487187 & 0.011507 \\ 7 & 2.83891 & -0.931664 & -0.006693 \\ 17 & 0.400575 & 0.969362 & 2.347839 \\ 17 & -0.386558 & -0.707395 & -2.164511\end{array}$




$\begin{array}{llll}17 & -2.25767 & 1.063645 & 0.139025 \\ 7 & -0.767294 & -1.531227 & 0.844255 \\ 6 & -1.286489 & -2.475632 & 1.284819 \\ 6 & -1.986757 & -3.632425 & 1.83514 \\ 1 & -3.06712 & -3.510939 & 1.694388 \\ 1 & -1.668877 & -4.551972 & 1.330165 \\ 1 & -1.781923 & -3.728358 & 2.907681\end{array}$

12

ruco-n2 $(\mathrm{tCO}) \mathrm{Cl} 3(\mathrm{NH} 3) \quad \mathrm{E}(\mathrm{RB} 3 \mathrm{LYP})=-1755.28660290$

$\begin{array}{llll}6 & 1.916226 & 0.148956 & -0.436295 \\ 8 & 3.051171 & 0.280158 & -0.766367 \\ 44 & 0.168933 & -0.07726 & 0.071122 \\ 7 & 0.574698 & -0.204603 & 1.952873 \\ 7 & 0.80322 & -0.273027 & 3.072442 \\ 17 & -0.057099 & 2.30696 & 0.333181 \\ 17 & -0.415452 & -0.045144 & -2.269009 \\ 17 & -2.186779 & -0.570114 & 0.746893 \\ 7 & 0.103196 & -2.208503 & -0.252715 \\ 1 & 0.856261 & -2.767152 & 0.147886 \\ 1 & -0.816528 & -2.426426 & 0.1542 \\ 1 & 0.06775 & -2.274324 & -1.276156\end{array}$

24

ruco-n2(tCO)Cl(CH3CN)3 E(RB3LYP $)=-1176.15136859$

$\begin{array}{llll}6 & -1.899118 & 2.27629 & 0.47216\end{array}$

$\begin{array}{llll}8 & -2.309329 & 3.316454 & 0.809742\end{array}$

$\begin{array}{llll}44 & -1.275803 & 0.597468 & -0.112141\end{array}$

$\begin{array}{llll}7 & 0.175445 & 0.486945 & 1.265848\end{array}$

$\begin{array}{llll}7 & 1.000702 & 0.418197 & 2.044918\end{array}$

$\begin{array}{llll}17 & -2.953634 & 0.643177 & -1.764583\end{array}$

$\begin{array}{llll}7 & -2.59498 & -0.341121 & 1.102146\end{array}$

$\begin{array}{llll}6 & -3.413934 & -0.862093 & 1.74711\end{array}$

$\begin{array}{llll}6 & -4.462978 & -1.497693 & 2.5383\end{array}$

$\begin{array}{llll}1 & -5.336267 & -1.699209 & 1.907214\end{array}$

$\begin{array}{llll}1 & -4.108186 & -2.44383 & 2.962335\end{array}$

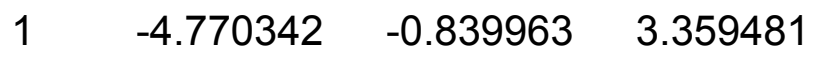

$\begin{array}{llll}7 & -0.039697 & 1.491711 & -1.441654\end{array}$

$\begin{array}{llll}6 & 0.605879 & 2.020407 & -2.255157\end{array}$

$\begin{array}{llll}6 & 1.387205 & 2.695782 & -3.2866\end{array}$

$\begin{array}{llll}1 & 2.40757 & 2.297773 & -3.322417\end{array}$

$\begin{array}{llll}1 & 0.917022 & 2.552277 & -4.266292\end{array}$

$\begin{array}{llll}1 & 1.438219 & 3.770889 & -3.079186\end{array}$

$\begin{array}{llll}7 & -0.701253 & -1.26365 & -0.877634\end{array}$ 


$\begin{array}{llll}6 & -0.450129 & -2.291879 & -1.367019 \\ 6 & -0.158688 & -3.574909 & -1.999554 \\ 1 & -0.223157 & -4.38732 & -1.266949 \\ 1 & -0.880219 & -3.767157 & -2.801949 \\ 1 & 0.849879 & -3.570654 & -2.428115\end{array}$

\begin{tabular}{llll}
18 & \multicolumn{3}{l}{ E } \\
ruco-n2(tCO)Cl(NH3)3 & \\
6 & -1.844943 & -0.956872 & -1.343664 \\
8 & -2.485836 & -1.473443 & -2.179549 \\
44 & -0.845152 & -0.096239 & -0.013757 \\
7 & 0.303357 & -1.658107 & 0.32657 \\
7 & 0.974308 & -2.559203 & 0.529859 \\
17 & -2.12011 & 1.873712 & -0.283417 \\
7 & 0.482776 & 0.722665 & -1.502589 \\
1 & -0.004211 & 1.566455 & -1.833224 \\
1 & 1.406189 & 0.990804 & -1.148766 \\
7 & 0.218958 & 1.080493 & 1.520682 \\
1 & 1.21657 & 1.221189 & 1.332367 \\
1 & -0.263321 & 1.989098 & 1.477229 \\
1 & 0.140558 & 0.706625 & 2.47178 \\
1 & 0.624438 & 0.090649 & -2.296399 \\
7 & -2.290971 & -0.66979 & 1.480983 \\
1 & -1.930378 & -0.680228 & 2.440029 \\
1 & -3.024344 & 0.048236 & 1.410004 \\
1 & -2.707896 & -1.586994 & 1.294777
\end{tabular}

\begin{tabular}{llll}
14 & \multicolumn{1}{l}{} \\
ruco-n2(tCO)F3(CH3CN) & $E(\mathrm{RB} 3 L Y P)=-750.279466781$ \\
6 & 0.538459 & 1.85338 & -0.678365 \\
8 & 0.862846 & 2.903858 & -1.132448 \\
44 & 0.014658 & 0.248037 & 0.019258 \\
7 & 1.803835 & -0.409682 & 0.087264 \\
7 & 2.872827 & -0.837724 & 0.140484 \\
9 & -0.278229 & -0.616765 & -1.786624 \\
9 & 0.121368 & 0.870511 & 1.943682 \\
9 & -1.886375 & 0.788209 & 0.009615 \\
7 & -0.745816 & -1.553147 & 0.830796 \\
6 & -1.279267 & -2.485794 & 1.280284 \\
6 & -1.987566 & -3.631906 & 1.844558 \\
1 & -2.976241 & -3.319013 & 2.199876 \\
1 & -2.119267 & -4.411696 & 1.085521 \\
1 & -1.429231 & -4.052294 & 2.689077
\end{tabular}




\begin{tabular}{llll}
24 & \multicolumn{4}{l}{} \\
ruco-n2(tCO)F(CH3CN)3 & $E(R B 3 L Y P)=-815.767969204$ \\
6 & -1.853628 & 2.275324 & 0.518798 \\
8 & -2.253205 & 3.316153 & 0.868757 \\
44 & -1.245743 & 0.597595 & -0.081944 \\
7 & 1.019644 & 0.419849 & 2.062153 \\
7 & 0.190465 & 0.49649 & 1.285988 \\
9 & -2.621921 & 0.581203 & -1.47483 \\
7 & -2.586343 & -0.338817 & 1.106018 \\
6 & -3.429379 & -0.857272 & 1.721323 \\
6 & -4.503796 & -1.489942 & 2.480068 \\
1 & -5.358799 & -1.688179 & 1.823483 \\
1 & -4.164674 & -2.437546 & 2.91355 \\
1 & -4.832998 & -0.831888 & 3.292465 \\
7 & -0.03775 & 1.492067 & -1.433161 \\
6 & 0.578003 & 2.018652 & -2.270812 \\
6 & 1.327494 & 2.690419 & -3.327868 \\
1 & 2.352568 & 2.306335 & -3.378952 \\
1 & 0.839877 & 2.526194 & -4.295669 \\
1 & 1.367336 & 3.768956 & -3.136624 \\
7 & -0.681915 & -1.261334 & -0.857736 \\
6 & -0.445627 & -2.286932 & -1.359581 \\
6 & -0.16708 & -3.567676 & -2.002439 \\
1 & -0.258367 & -4.386812 & -1.280255 \\
1 & -0.878432 & -3.738017 & -2.818681 \\
1 & 0.848475 & -3.576931 & -2.414045
\end{tabular}

18 ruco-n2 $(\mathrm{tCO}) \mathrm{F}(\mathrm{NH} 3) 3 \quad \mathrm{E}(\mathrm{RB} 3 \mathrm{LYP})=-587.236776347$

$\begin{array}{llll}6 & -1.80979 & -1.004464 & -1.335391 \\ 8 & -2.444454 & -1.521916 & -2.176431 \\ 44 & -0.813316 & -0.140648 & -0.005079 \\ 7 & 0.307431 & -1.70722 & 0.312234 \\ 7 & 0.971326 & -2.61404 & 0.518023 \\ 9 & -1.826567 & 1.55239 & -0.160675 \\ 7 & 0.445062 & 0.760765 & -1.490411 \\ 1 & -0.076934 & 1.625715 & -1.689778 \\ 1 & 1.393621 & 0.996665 & -1.184198 \\ 7 & 0.207103 & 1.076853 & 1.508217 \\ 1 & 1.197072 & 1.267225 & 1.32546 \\ 1 & -0.334832 & 1.947567 & 1.411556 \\ 1 & 0.136393 & 0.734003 & 2.471144 \\ 1 & 0.523541 & 0.2127 & -2.351932 \\ 7 & -2.301762 & -0.615864 & 1.465479\end{array}$




$\begin{array}{llll}1 & -1.968153 & -0.667617 & 2.43259 \\ 1 & -2.94001 & 0.185918 & 1.365445 \\ 1 & -2.815737 & -1.478984 & 1.266663\end{array}$

9 ruco-n2F4(tCO) $\quad E(R B 3 L Y P)=-717.355034016$

$\begin{array}{llll}6 & 0.781126 & 1.778168 & -0.708666\end{array}$

$\begin{array}{llll}8 & 1.314495 & 2.770613 & -1.150122\end{array}$

$\begin{array}{llll}44 & -0.013782 & 0.285566 & -0.042102\end{array}$

$\begin{array}{llll}7 & 1.503661 & -0.791459 & -0.348469\end{array}$

$\begin{array}{llll}7 & 2.415494 & -1.484667 & -0.526882\end{array}$

$\begin{array}{llll}9 & -0.788757 & -0.226632 & -1.857767\end{array}$

$\begin{array}{llll}9 & -1.684963 & 1.296078 & 0.331809\end{array}$

$\begin{array}{llll}9 & -0.91823 & -1.358011 & 0.709181\end{array}$

$\begin{array}{llll}9 & 0.601726 & 0.700727 & 1.857361\end{array}$

Mixed $\mathrm{Ru}(\mathrm{tCO})-\mathrm{N}_{\mathbf{2}}$ species [Fig. 16]

\begin{tabular}{llll}
21 & \multicolumn{2}{c}{$E(\mathrm{RB} 3 \mathrm{LYP})=-543.566129703$} \\
rutco-n2(NH3)4 & \multicolumn{2}{c}{-0.253764} \\
6 & 0.696251 & 3.420092 & -0.640052 \\
8 & 1.259968 & 4.370033 & -0.6405 \\
44 & -0.20718 & 1.895795 & 0.366128 \\
7 & -1.203768 & 0.210969 & 1.048956 \\
7 & -1.748973 & -0.711288 & 1.420557 \\
7 & 1.354594 & 0.599839 & -0.433202 \\
7 & -1.810856 & 3.125829 & 1.187369 \\
1 & 1.009195 & -0.346996 & -0.633956 \\
1 & 2.139649 & 0.494734 & 0.221284 \\
1 & 1.753232 & 0.970995 & -1.304181 \\
1 & -2.541262 & 3.319849 & 0.49146 \\
1 & -2.276223 & 2.674444 & 1.984815 \\
1 & -1.469538 & 4.039436 & 1.510066 \\
7 & 0.849344 & 2.002584 & 2.271068 \\
1 & 0.445629 & 2.712391 & 2.89466 \\
1 & 1.836943 & 2.257961 & 2.148648 \\
1 & 0.825563 & 1.111074 & 2.781667 \\
7 & -1.304413 & 1.734489 & -1.512697 \\
1 & -2.171992 & 1.192916 & -1.412165 \\
1 & -1.56086 & 2.655744 & -1.888256 \\
1 & -0.750273 & 1.271287 & -2.243492
\end{tabular}




\begin{tabular}{llll}
\multicolumn{1}{l}{ rutco-n2(Acac)2 } & \multicolumn{2}{c}{$\mathrm{E}(\mathrm{RB} 3 \mathrm{LYP})=-1008.20413169$} \\
6 & 2.739776 & 4.362743 & 0.910495 \\
8 & 3.592222 & 5.179808 & 0.903768 \\
44 & 1.407517 & 3.085005 & 0.920777 \\
7 & -0.096132 & 1.642628 & 0.932159 \\
7 & -0.912917 & 0.859171 & 0.938395 \\
8 & 2.623621 & 1.716324 & 1.872248 \\
6 & 2.906754 & 1.448181 & -1.125119 \\
6 & 3.176023 & 1.149783 & -2.586994 \\
1 & 3.918761 & 0.357641 & -2.716025 \\
1 & 2.241697 & 0.855391 & -3.080617 \\
1 & 3.529829 & 2.060701 & -3.086245 \\
6 & 3.624353 & 0.758329 & -0.117592 \\
6 & 3.455915 & 0.897394 & 1.281792 \\
6 & 4.275542 & 0.046835 & 2.232182 \\
1 & 4.929338 & -0.651199 & 1.701908 \\
1 & 3.605222 & -0.515185 & 2.894082 \\
1 & 4.360674 & 0.03667 & -0.450897 \\
1 & 4.885713 & 0.698037 & 2.870777 \\
8 & 0.082488 & 4.348813 & -0.030369 \\
8 & 0.714858 & 3.706305 & 2.762413 \\
6 & -0.696853 & 5.218756 & 0.559349 \\
6 & -1.517562 & 6.067826 & -0.391434 \\
1 & -2.200356 & 6.737975 & 0.138295 \\
1 & -0.844879 & 6.663943 & -1.021209 \\
1 & -2.093006 & 5.417832 & -1.06179 \\
6 & -0.819361 & 5.402397 & 1.958355 \\
6 & -0.152752 & 4.664006 & 2.966484 \\
6 & -0.430671 & 4.954818 & 4.428276 \\
1 & -1.193603 & 5.727571 & 4.557009 \\
1 & 0.496246 & 5.278042 & 4.918816 \\
1 & -1.507778 & 6.170178 & 2.291017 \\
1 & -0.756211 & 4.035714 & 4.930819 \\
8 & 1.986513 & 2.35524 & -0.920312 \\
& & & \\
& & & \\
1 & &
\end{tabular}

\begin{tabular}{llll}
12 & & & \\
rutco-n2aF3 & \multicolumn{2}{l}{$\mathrm{E}(\mathrm{RB} 3 \mathrm{LYP})=-674.092048815$} \\
6 & -0.427927 & 2.197424 & -0.260589 \\
8 & -0.646658 & 3.373345 & -0.327915 \\
44 & -0.104517 & 0.415865 & -0.149162 \\
7 & 0.237281 & -1.602389 & 0.153296 \\
7 & 0.404736 & -2.693217 & 0.41709 \\
9 & -2.067082 & -0.117103 & -0.225718 \\
9 & 1.960451 & 0.566629 & -0.379917 \\
9 & -0.159909 & 0.432645 & 1.867888
\end{tabular}




$\begin{array}{llll}7 & 0.210433 & 0.206816 & -2.279584 \\ 1 & -0.230608 & 0.943813 & -2.826606 \\ 1 & 1.232865 & 0.32499 & -2.188276 \\ 1 & -0.062937 & -0.709379 & -2.634139\end{array}$

\begin{tabular}{llll}
29 & & & \\
rutco-n2(CH3CN)4 & \multicolumn{2}{l}{$\mathrm{E}(\mathrm{RB} 3 \mathrm{LYP})=-848.334467372$} \\
6 & 0.05655 & 1.938979 & 0.00649 \\
8 & 0.076732 & 3.102156 & 0.007119 \\
44 & 0.023606 & 0.051583 & 0.005459 \\
7 & -0.013387 & -2.051544 & 0.004537 \\
7 & -0.033318 & -3.180029 & 0.004159 \\
7 & -1.437126 & 0.048384 & -1.409188 \\
7 & -1.390644 & 0.046039 & 1.466571 \\
7 & 1.483918 & -0.004497 & 1.41945 \\
7 & 1.436838 & -0.002329 & -1.455656 \\
6 & -2.274716 & 0.054742 & -2.222103 \\
6 & -2.202037 & 0.05073 & 2.305644 \\
6 & 2.322598 & -0.028495 & 2.23091 \\
6 & 2.247874 & -0.025282 & -2.294776 \\
6 & -3.321101 & 0.067586 & -3.239049 \\
6 & -3.215935 & 0.061394 & 3.355018 \\
6 & 3.371617 & -0.053826 & 3.244914 \\
6 & 3.261434 & -0.04927 & -3.344253 \\
1 & -4.283008 & -0.226966 & -2.802859 \\
1 & -3.424632 & 1.073064 & -3.664608 \\
1 & -3.077363 & -0.630899 & -4.048428 \\
1 & -2.950597 & -0.644148 & 4.151357 \\
1 & -3.300941 & 1.064257 & 3.790701 \\
1 & -4.192524 & -0.22524 & 2.947118 \\
1 & 4.139075 & -0.792184 & 2.983833 \\
1 & 3.847031 & 0.931287 & 3.324176 \\
1 & 2.952612 & -0.31815 & 4.223087 \\
1 & 2.972614 & -0.746274 & -4.13994 \\
1 & 4.227997 & -0.367834 & -2.936153 \\
1 & 3.379687 & 0.949813 & -3.780871
\end{tabular}

\footnotetext{
23

rutco-n2(CH3CN)(NH3)3 $E(\mathrm{RB} 3 \mathrm{LYP})=-619.763476588$

$\begin{array}{llll}6 & -1.606478 & 1.238667 & -1.172032\end{array}$

$\begin{array}{llll}8 & -2.207308 & 1.897686 & -1.926953\end{array}$

$\begin{array}{llll}44 & -0.652962 & 0.183651 & 0.057334\end{array}$

$\begin{array}{llll}7 & 0.382094 & -0.975243 & 1.44412\end{array}$

$\begin{array}{llll}7 & 0.914793 & -1.594087 & 2.228749\end{array}$ 


$\begin{array}{llll}7 & 0.88233 & -0.236177 & -1.415831 \\ 1 & 1.438067 & 0.598026 & -1.640132 \\ 1 & 1.540713 & -0.957734 & -1.097531 \\ 7 & 0.445293 & 1.922449 & 0.75949 \\ 1 & 1.350582 & 1.681342 & 1.18048 \\ 1 & 0.622606 & 2.61568 & 0.023346 \\ 7 & -1.756254 & -1.58569 & -0.550164 \\ 1 & -1.920853 & -1.636862 & -1.562134 \\ 1 & -1.294149 & -2.458846 & -0.269781 \\ 1 & -2.678093 & -1.570238 & -0.097639 \\ 1 & -0.111771 & 2.39207 & 1.483398 \\ 1 & 0.483074 & -0.564023 & -2.303502 \\ 7 & -2.069593 & 0.541385 & 1.489418 \\ 6 & -2.889074 & 0.756489 & 2.297629 \\ 6 & -3.91226 & 1.028957 & 3.302768 \\ 1 & -4.716416 & 0.285186 & 3.243232 \\ 1 & -3.480175 & 0.993228 & 4.310367 \\ 1 & -4.347615 & 2.023261 & 3.143324\end{array}$

\begin{tabular}{llll}
19 & \multicolumn{3}{l}{$\mathrm{l}(\mathrm{C})$} \\
rutco-n2Cl2(CH3CN)2 & $\mathrm{E}(\mathrm{RB} 3 \mathrm{LYP})=-1503.84743302$ \\
6 & -2.798498 & 0.685711 & -1.879723 \\
8 & -3.655704 & 0.78769 & -2.676925 \\
44 & -1.472837 & 0.539743 & -0.590425 \\
7 & 0.513323 & 0.44294 & 1.929138 \\
7 & -0.149145 & 0.459445 & 1.012999 \\
17 & -2.059859 & 2.717718 & 0.19459 \\
17 & -3.05315 & -0.52043 & 0.852625 \\
7 & -0.079107 & 1.466501 & -1.738902 \\
6 & 0.683675 & 2.087957 & -2.368156 \\
6 & 1.609302 & 2.916114 & -3.138006 \\
1 & 2.648088 & 2.671025 & -2.888421 \\
1 & 1.462285 & 2.76046 & -4.213054 \\
1 & 1.436778 & 3.974916 & -2.91228 \\
7 & -0.9405 & -1.330993 & -1.170799 \\
6 & -0.713205 & -2.442636 & -1.447535 \\
6 & -0.479948 & -3.850647 & -1.761182 \\
1 & -1.117415 & -4.482261 & -1.131482 \\
1 & -0.717027 & -4.054625 & -2.81177 \\
1 & 0.566852 & -4.120762 & -1.580448
\end{tabular}

14 rutco-n2Cl3(CH3CN) $\quad E(R B 3 L Y P)=-1831.44577802$ $\begin{array}{llll}6 & -1.945488 & 1.030596 & 0.149165\end{array}$ 


$\begin{array}{llll}8 & -3.001536 & 1.562464 & 0.116835 \\ 44 & -0.292426 & 0.221716 & 0.19111 \\ 7 & 1.624808 & -0.534684 & 0.171137 \\ 7 & 2.693958 & -0.907552 & 0.142018 \\ 17 & 0.657924 & 2.231197 & -0.735142 \\ 17 & 0.126846 & 0.967862 & 2.481303 \\ 17 & -0.60544 & -0.761619 & -2.024379 \\ 7 & -1.010036 & -1.473729 & 0.95897 \\ 6 & -1.432947 & -2.466202 & 1.408896 \\ 6 & -1.971603 & -3.701809 & 1.972654 \\ 1 & -3.003518 & -3.857632 & 1.634626 \\ 1 & -1.372139 & -4.563281 & 1.653585 \\ 1 & -1.966707 & -3.660757 & 3.068862\end{array}$

12

\begin{tabular}{llll} 
rutco-n2Cl3(NH3) & \multicolumn{2}{l}{$\mathrm{E}(\mathrm{RB} 3 \mathrm{LYP})=-1755.27702206$} \\
6 & -1.427877 & 1.473634 & -1.03334 \\
8 & -1.90658 & 2.29472 & -1.744989 \\
44 & -0.670836 & 0.225972 & 0.078588 \\
7 & 0.282396 & -1.019242 & 1.3905 \\
7 & 0.848111 & -1.655843 & 2.141115 \\
17 & -2.45651 & 0.425797 & 1.746306 \\
17 & 0.93653 & -0.511743 & -1.614142 \\
17 & 0.725478 & 1.981477 & 0.995852 \\
7 & -1.86131 & -1.419976 & -0.650335 \\
1 & -2.428464 & -1.111685 & -1.439502 \\
1 & -1.211156 & -2.147772 & -0.956526 \\
1 & -2.460265 & -1.697394 & 0.132793
\end{tabular}

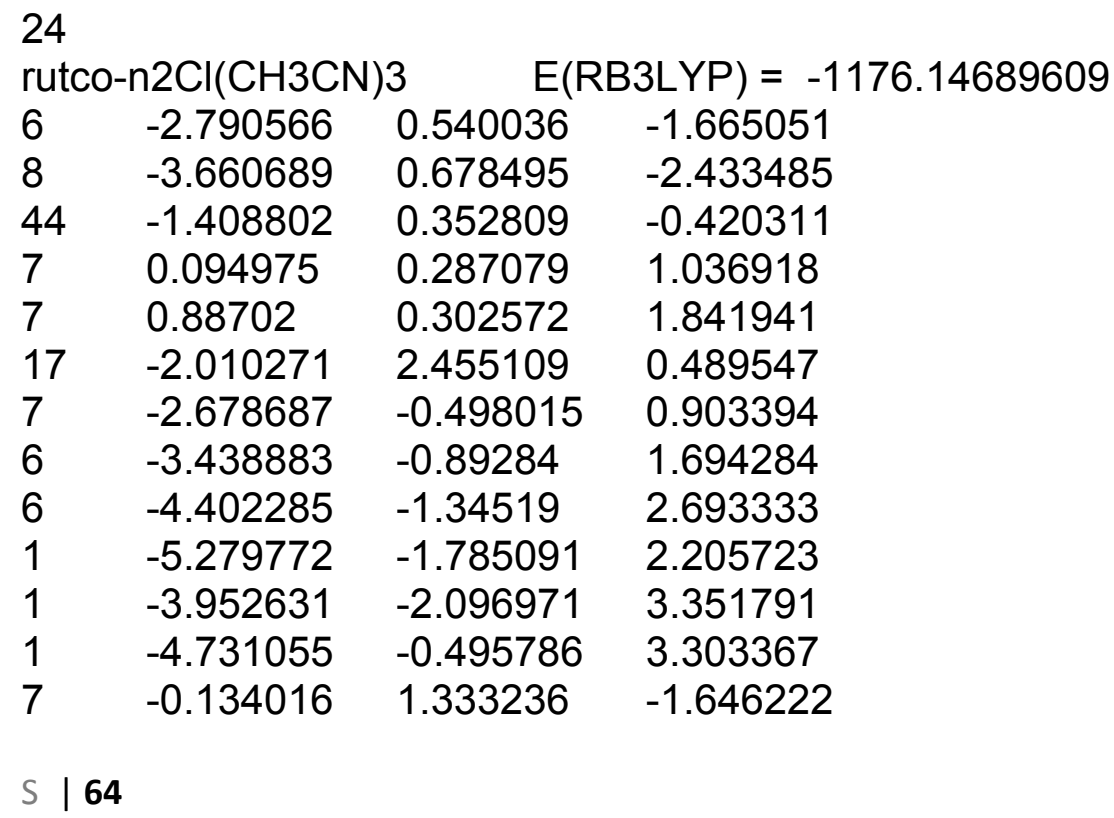




$\begin{array}{llll}6 & 0.562493 & 1.986414 & -2.31519 \\ 6 & 1.414756 & 2.840831 & -3.136456 \\ 1 & 2.472699 & 2.633319 & -2.939633 \\ 1 & 1.215712 & 2.671546 & -4.200816 \\ 1 & 1.215873 & 3.894025 & -2.90632 \\ 7 & -0.818102 & -1.47754 & -1.148214 \\ 6 & -0.491225 & -2.516514 & -1.570606 \\ 6 & -0.087863 & -3.815271 & -2.104879 \\ 1 & -0.352497 & -4.616482 & -1.405366 \\ 1 & -0.591749 & -4.006027 & -3.059538 \\ 1 & 0.995196 & -3.841669 & -2.27106\end{array}$

$\begin{array}{llll}18 & & & \\ \text { rutco-n2Cl(NH3)3 } & \mathrm{E}(\mathrm{RB} 3 \mathrm{LYP})= & -947.601228258 \\ 6 & -2.127933 & 1.389041 & -0.474603 \\ 8 & -2.907632 & 2.22893 & -0.737406 \\ 44 & -0.914216 & 0.050961 & -0.035395 \\ 7 & 0.351679 & -1.471596 & 0.543615 \\ 7 & 0.998984 & -2.326693 & 0.912334 \\ 17 & -2.395629 & -0.724609 & 1.65849 \\ 7 & 0.528012 & 0.689632 & -1.57778 \\ 1 & 0.82626 & 1.656463 & -1.415486 \\ 1 & 1.365132 & 0.098264 & -1.58851 \\ 7 & -0.051062 & 1.291372 & 1.514256 \\ 1 & 0.915384 & 1.057232 & 1.761235 \\ 1 & -0.104849 & 2.298781 & 1.338867 \\ 7 & -1.930748 & -1.351203 & -1.333072 \\ 1 & -2.392311 & -0.931559 & -2.145068 \\ 1 & -1.350471 & -2.130728 & -1.657692 \\ 1 & -2.654682 & -1.72205 & -0.700463 \\ 1 & -0.658768 & 1.066557 & 2.315411 \\ 1 & 0.101666 & 0.650557 & -2.508744\end{array}$

14

rutco-n2F3(CH3CN) E(RB3LYP $)=-750.268000795$

$\begin{array}{llll}6 & 0.491509 & 1.909002 & 0.057011 \\ 8 & 0.521695 & 3.100433 & 0.084438 \\ 44 & 0.446511 & 0.092618 & 0.015895 \\ 7 & 0.530956 & -2.006914 & -0.031722 \\ 7 & 0.642187 & -3.132354 & -0.058297 \\ 9 & 0.449945 & -0.118084 & 2.037956 \\ 9 & 0.460082 & -0.028978 & -2.014034 \\ 9 & 2.431278 & -0.05908 & 0.017587 \\ 7 & -1.519585 & 0.076519 & 0.006925\end{array}$




$\begin{array}{llll}6 & -2.694103 & 0.059754 & -0.001941 \\ 6 & -4.155513 & 0.05807 & -0.022972 \\ 1 & -4.552084 & -0.803804 & 0.529578 \\ 1 & -4.547034 & 0.970456 & 0.445742 \\ 1 & -4.536387 & 0.011273 & -1.052172\end{array}$

9

\begin{tabular}{llll} 
rutco-n2F4 & \multicolumn{2}{l}{$E($ RB3LYP $)=-717.324978385$} \\
6 & -1.919609 & 0.00348 & 0.001147 \\
8 & -3.130259 & 0.0048 & 0.00218 \\
44 & -0.116553 & 0.001393 & -0.000375 \\
7 & 1.93783 & -0.001188 & -0.002019 \\
7 & 3.076676 & -0.00268 & -0.002864 \\
9 & 0.044897 & 1.446247 & -1.462451 \\
9 & 0.041369 & -1.460589 & -1.445689 \\
9 & 0.043807 & -1.443856 & 1.461421 \\
9 & 0.047376 & 1.463029 & 1.444657
\end{tabular}

24

rutco-n2F $(\mathrm{CH} 3 \mathrm{CN}) 3 \mathrm{E}(\mathrm{RB} 3 \mathrm{LYP})=-815.761900323$

$6-2.769327$

$8-3.634775$

$44 \quad-1.398364$

0.479821

$-1.689146$

$7 \quad 0.108468$

$0.601691-2.467531$

$0.314415 \quad-0.437279$

$7 \quad 0.891321$

$0.258733 \quad 1.031285$

$9 \quad-1.8375$

$0.29738 \quad 1.843585$

$7 \quad-2.676892$

$2.057404 \quad 0.376573$

$6 \quad-3.444878$

$\begin{array}{ll}-0.500481 & 0.896247\end{array}$

$\begin{array}{ll}-0.853079 & 1.69967\end{array}$

$\begin{array}{llll}6 & -4.416351 & -1.258819 & 2.710793\end{array}$

$\begin{array}{llll}1 & -5.305534 & -1.688063 & 2.235015\end{array}$

$\begin{array}{llll}1 & -3.983372 & -2.006825 & 3.384607\end{array}$

$\begin{array}{llll}1 & -4.722693 & -0.388587 & 3.30279\end{array}$

$\begin{array}{llll}7 & -0.136967 & 1.328355 & -1.64459\end{array}$

$\begin{array}{llll}6 & 0.542809 & 2.019529 & -2.292494\end{array}$

$\begin{array}{llll}6 & 1.374768 & 2.914372 & -3.091162\end{array}$

$\begin{array}{llll}1 & 2.437324 & 2.724597 & -2.901395\end{array}$

$\begin{array}{llll}1 & 1.17766 & 2.768857 & -4.159416\end{array}$

$\begin{array}{llll}1 & 1.153643 & 3.956553 & -2.833141\end{array}$

$\begin{array}{llll}7 & -0.820697 & -1.504366 & -1.170338\end{array}$

$\begin{array}{lllll}6 & -0.491495 & -2.543598 & -1.590959\end{array}$

$\begin{array}{llll}6 & -0.083881 & -3.842584 & -2.121614\end{array}$

$\begin{array}{llll}1 & -0.345721 & -4.643192 & -1.4203\end{array}$

$\begin{array}{llll}1 & -0.587121 & -4.037803 & -3.075745\end{array}$

$\begin{array}{llll}1 & 0.999205 & -3.866236 & -2.288304\end{array}$ 


\begin{tabular}{llll}
18 & & & \\
rutco-n2F(NH3)3 & \multicolumn{2}{l}{$\mathrm{E}(\mathrm{RB} 3 \mathrm{LYP})=-587.217953454$} \\
6 & -2.066091 & 1.419942 & -0.543336 \\
8 & -2.815272 & 2.27719 & -0.844101 \\
44 & -0.887596 & 0.069261 & -0.068886 \\
7 & 0.379831 & -1.460222 & 0.513808 \\
7 & 1.02479 & -2.316255 & 0.883119 \\
9 & -2.084935 & -0.625891 & 1.366644 \\
7 & 0.544292 & 0.707321 & -1.608195 \\
1 & 0.79342 & 1.693796 & -1.486739 \\
1 & 1.409376 & 0.158702 & -1.574173 \\
7 & -0.116982 & 1.24937 & 1.560248 \\
1 & 0.847681 & 1.054199 & 1.843108 \\
1 & -0.228217 & 2.262698 & 1.470413 \\
7 & -1.981767 & -1.36053 & -1.251646 \\
1 & -2.534256 & -0.97671 & -2.022802 \\
1 & -1.446106 & -2.159155 & -1.603757 \\
1 & -2.621074 & -1.681881 & -0.507993 \\
1 & -0.76083 & 0.904745 & 2.289326 \\
1 & 0.14255 & 0.602771 & -2.545052
\end{tabular}

